\title{
The High-Acceptance Dielectron Spectrometer HADES
}

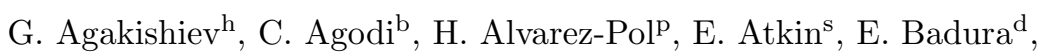
A. Balanda ${ }^{\mathrm{c}, \mathrm{y}}$, A. Bassi ${ }^{\mathrm{i}}$, R. Bassini ${ }^{\mathrm{i}}$, G. Bellia ${ }^{\mathrm{b}, \mathrm{v}}$, D. Belver ${ }^{\mathrm{p}}$, A.V Belyaev ${ }^{\mathrm{f}}$, M. Benovic ${ }^{\mathrm{a}}$, D. Bertini ${ }^{\mathrm{d}}$, J. Bielcik ${ }^{\mathrm{d}}$, M. Böhmer ${ }^{\text {, C. Boiano }}{ }^{\mathrm{i}}$, H. Bokemeyer ${ }^{\mathrm{d}}$, A. Bartolotti ${ }^{\mathrm{i}}$, J.L. Boyard ${ }^{\mathrm{n}}$, S. Brambilla ${ }^{\mathrm{i}}$, P. Braun-Munzinger ${ }^{\mathrm{t}, \mathrm{u}}$, P. Cabanelas ${ }^{p}$, E. Castrop ${ }^{p}$, V. Chepurnov ${ }^{f}$, S. Chernenkof ${ }^{f}$ T. Christ ${ }^{1}$, R. Coniglione ${ }^{\text {b }}$, L. Cosentino ${ }^{b}$, M. Dahlinger ${ }^{d}$, H.W. Daues ${ }^{d}$, M. Destefanis ${ }^{\text {h }}$, J. Díaz ${ }^{\mathrm{q}}$, F. Dohrmann ${ }^{\mathrm{e}}$, R. Dressler ${ }^{\mathrm{e}}$, I. Durán ${ }^{\mathrm{p}}$, A. Dybczak ${ }^{\mathrm{c}}$, T. Eberl ${ }^{\mathrm{l}}$, W. Enghardt ${ }^{\mathrm{e}}$, L. Fabbietti ${ }^{\mathrm{l}}$ O.V. Fateev ${ }^{\mathrm{f}}$, C. Fernandez ${ }^{\mathrm{p}}$, P. Finocchiaro ${ }^{\mathrm{b}}$, J. Friese ${ }^{\mathrm{l}}$, I. Fröhlich ${ }^{\mathrm{g}}$, B. Fuentes ${ }^{\mathrm{p}}$, T. Galatyuk ${ }^{\mathrm{d}}$, C. Garabatos ${ }^{\mathrm{d}}$, J.A. Garzón ${ }^{\mathrm{p}}$, B. Genolini ${ }^{\mathrm{n}}$, R. Gernhäuser ${ }^{\mathrm{l}}$, C. Gilardir ${ }^{\mathrm{h}}$, H. Gilg ${ }^{\mathrm{l}}$, M. Golubeva ${ }^{\mathrm{j}}$, D. González-Díaz ${ }^{\mathrm{d}}$, E. Grosse ${ }^{\mathrm{e}, \mathrm{w}}$, F. Guber ${ }^{\mathrm{j}}$, J. Hehner ${ }^{\mathrm{d}}$,

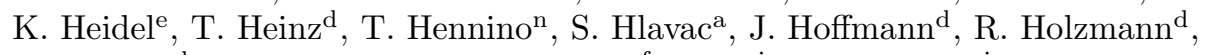
J. Homolka ${ }^{1}$, J. Hutsch ${ }^{\mathrm{e}}$, A.P. Ierusalimov ${ }^{\mathrm{f}}$, I. Iori ${ }^{\mathrm{i}, \mathrm{x}}$, A. Ivashkin ${ }^{\mathrm{j}}$, M. Jaskula ${ }^{\mathrm{c}}$, J. C. Jourdain ${ }^{\text {n, M. Jurkovic }}{ }^{1}$, B. Kämpfer ${ }^{\mathrm{e}, \mathrm{w}}$, M. Kajetanowicz ${ }^{\mathrm{c}}$, K. Kanaki ${ }^{\mathrm{e}}$,

T. Karavicheva ${ }^{j}$, A. Kastenmüller ${ }^{\mathrm{l}}$, L. Kidon ${ }^{\mathrm{c}}$, P. Kienle ${ }^{\mathrm{l}}$, D. Kirschner ${ }^{\mathrm{h}}$,

I. Koenig d , W. Koenigd ${ }^{d}$ H.J. Körner ${ }^{1}$, B.W. Kolb ${ }^{d}$, U. Kopf ${ }^{d}$, K. Korcyl ${ }^{c}$,

R. Kotte ${ }^{\mathrm{e}}$, A. Kozuch ${ }^{\mathrm{c}, \mathrm{y}}$, F. Krizek ${ }^{\mathrm{o}}$, R. Krücken ${ }^{\mathrm{l}}$, W. Kühn ${ }^{\mathrm{h}}$, A. Kugler ${ }^{\mathrm{e}}$,

R. Kulessac ${ }^{\text {, A. Kurepin }}{ }^{\mathrm{j}}$, T. Kurtukian-Nieto ${ }^{\mathrm{p}}$, S. Lang ${ }^{\mathrm{d}}$, J. S. Lange ${ }^{\mathrm{h}}$, K.

Lapidus $^{j}$, J. Lehnert ${ }^{\mathrm{h}}$, U. Leinberger ${ }^{\mathrm{d}}$, C. Lichtblau ${ }^{\mathrm{h}}$, E. Lins $^{\mathrm{h}}$, C. Lippmann ${ }^{\mathrm{g}}$, M. Lorentz ${ }^{\mathrm{g}}$, D. Magestro ${ }^{\mathrm{d}}$, L. Maier ${ }^{\mathrm{l}}$, P. Maier-Komor ${ }^{\mathrm{l}}$, C. Maiolino ${ }^{\mathrm{b}}$,

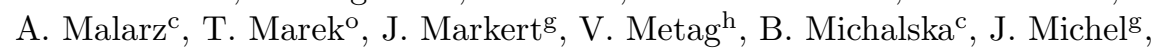
E. Migneco ${ }^{\text {b,v }}$, D. Mishra ${ }^{\text {h }}$, E. Morinière ${ }^{n}$, J. Mousa $^{m}$, M. Münch $^{\mathrm{d}}$, C. Müntz $^{\mathrm{g}}$, L. Naumann ${ }^{\mathrm{e}}$, A. Nekhaev ${ }^{\mathrm{k}}$, W. Niebur ${ }^{\mathrm{d}}$, J. Novotny ${ }^{\mathrm{o}}$, R. Novotny ${ }^{\mathrm{h}}$, W. Ott ${ }^{\mathrm{d}}$, J. Otwinowski ${ }^{\mathrm{c}}$, Y. C. Pachmayer ${ }^{\mathrm{g}}$, M. Palka ${ }^{\mathrm{d}, \mathrm{c}}$, Y. Parpottas ${ }^{\mathrm{m}}$, V. Pechenov ${ }^{\mathrm{h}}$, O. Pechenova ${ }^{\text {h }}$, T. Pérez Cavalcanti ${ }^{\text {h }}$, M. Petri ${ }^{\text {h }}$, P. Piattelli ${ }^{\text {b }}$, J. Pietraszko ${ }^{\text {, }}$, R. Pleskac ${ }^{\mathrm{o}}$, M. Ploskon ${ }^{\mathrm{c}}$, V. Pospísil ${ }^{\mathrm{o}}, \mathrm{J}_{\text {. Pouthas }}^{\mathrm{n}}$, W. Prokopowicz ${ }^{\mathrm{c}}$, W. Przygoda ${ }^{\mathrm{c}, \mathrm{y}}$, B. Ramstein ${ }^{\mathrm{n}}$, A. Reshetin ${ }^{\mathrm{j}}$, J. Ritman $^{\mathrm{h}}$, G. Roche ${ }^{\mathrm{r}}$, G. Rodriguez-Prieto ${ }^{\mathrm{p}}$, K. Rosenkranz ${ }^{\mathrm{g}}$, P. Rosier ${ }^{\mathrm{n}}$, M. Roy-Stephan ${ }^{\mathrm{n}}$,

A. Rustamov ${ }^{\mathrm{d}}$, J. Sabin-Fernandez ${ }^{\mathrm{p}}$, A. Sadovsky ${ }^{\mathrm{j}}$, B. Sailer ${ }^{1}$, P. Salabura ${ }^{\mathrm{c}}$, C. Salz ${ }^{\text {, M. Sánchez }}{ }^{\mathrm{p}}$, P. Sapienza ${ }^{\mathrm{b}}$, D. Schäfer ${ }^{\mathrm{h}}$, R.M. Schicker ${ }^{\mathrm{d}}$, A. Schmah ${ }^{\mathrm{d}, \mathrm{l}}$, H. Schön ${ }^{\mathrm{d}}$, W. Schön ${ }^{\mathrm{d}}$, C. Schroeder ${ }^{\mathrm{d}}$, S. Schroeder ${ }^{1}$, E. Schwab ${ }^{\text {d }}$, P. Senger ${ }^{d}$, K. Shileev ${ }^{j}$, R.S. Simon ${ }^{d}$, M. Skoda ${ }^{\mathrm{h}}$,

V. Smolyankin ${ }^{\mathrm{k}}$, L. Smykov ${ }^{\mathrm{f}}$, M. Sobiella ${ }^{\mathrm{e}}$, Yu.G. Sobolev ${ }^{\mathrm{o}}$, S. Spataro ${ }^{\mathrm{h}}$,

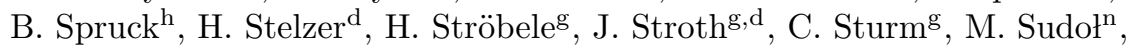

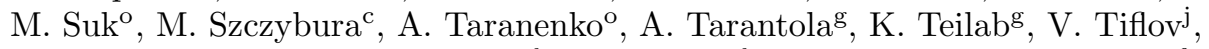

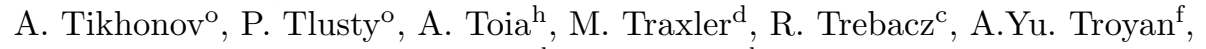

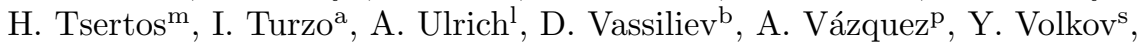
V. Wagner ${ }^{\mathrm{o}}$, C. Wallner ${ }^{\mathrm{l}}$, W. Walus ${ }^{\mathrm{c}}$, Y. Wang ${ }^{\mathrm{g}}$, M. Weber ${ }^{\mathrm{l}}$, J. Wieser ${ }^{\mathrm{l}}$, S. Winkler ${ }^{1}$, M. Wisniowski ${ }^{\mathrm{c}}$, T. Wojcik ${ }^{\mathrm{c}}, \mathrm{J}$. Wüstenfeld ${ }^{\mathrm{e}}$, S. Yurevich $^{\mathrm{d}}$, Y.V. Zanevsky ${ }^{\mathrm{f}}$, K. Zeitelhack ${ }^{1}$, A. Zentek ${ }^{\mathrm{g}}$, P. Zhou ${ }^{\mathrm{e}}$, D. Zovinec ${ }^{\mathrm{d}}$, P. Zumbruch ${ }^{\mathrm{d}}$

${ }^{a}$ Institute of Physics, Slovak Academy of Sciences, 84228 Bratislava, Slovakia ${ }^{b}$ Istituto Nazionale di Fisica Nucleare - Laboratori Nazionali del Sud, 95125 Catania, Italy 


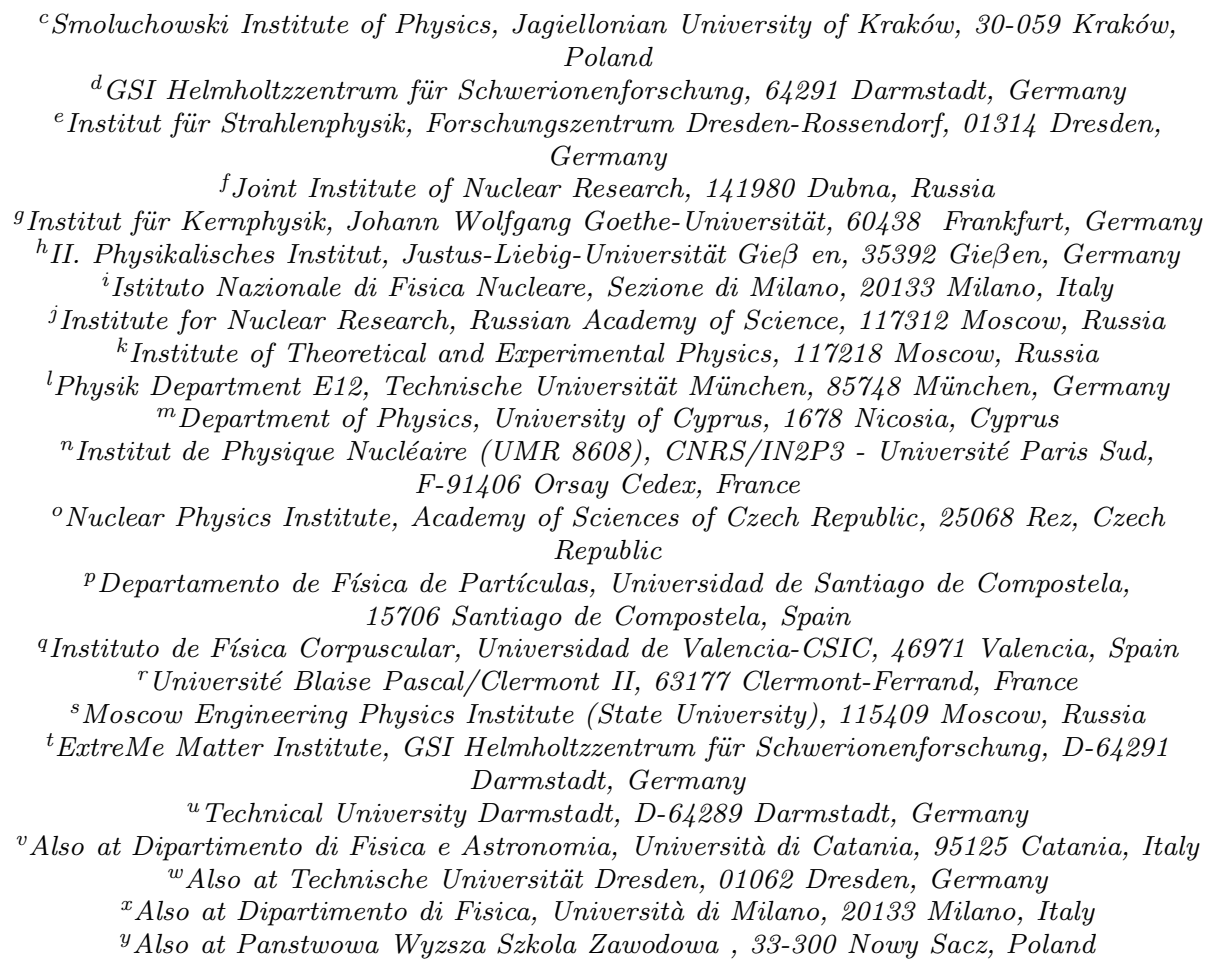

\begin{abstract}
HADES is a versatile magnetic spectrometer aimed at studying dielectron production in pion, proton and heavy-ion induced collisions. Its main features include a ring imaging gas Cherenkov detector for electron-hadron discrimination, a tracking system consisting of a set of 6 superconducting coils producing a toroidal field and drift chambers and a multiplicity and electron trigger array for additional electron-hadron discrimination and event characterization. A two-stage trigger system enhances events containing electrons. The physics program is focused on the investigation of hadron properties in nuclei and in the hot and dense hadronic matter. The detector system is characterized by an $85 \%$ azimuthal coverage over a polar angle interval from $18^{\circ}$ to $85^{\circ}$, a single electron efficiency of $50 \%$ and a vector meson mass resolution of $2.5 \%$. Identification of pions, kaons and protons is achieved combining time-of-flight and energy loss measurements over a large momentum range. This paper describes the main features and the performance of the detector system.
\end{abstract}

Key words: Spectrometer, Electron-positron pairs, Relativistic heavy-ion collisions, Hadron properties

PACS: 21.65, 24.85, 25.75, 29.30, 29.40 


\section{Introduction}

\subsection{Physics motivation}

A central topic of contemporary hadron physics is the investigation of hadronic matter. Theoretical models based on non-perturbative Quantum ChromoDynamics indicate that the properties of hadrons are modified, if the particles are embedded in a strongly interacting medium (for a theory overview see [1]).

The High-Acceptance DiElectron Spectrometer (HADES) in operation at the GSI Helmholtzzentrum für Schwerionenforschung has been specifically designed to study medium modifications of the light vector mesons $\rho, \omega, \phi$ [2]. Experimentally, these probes are well suited for two reasons. The vector mesons are short-lived with lifetimes comparable to the duration of the compression phase of relativistic heavy-ion reactions in the 1 to $2 \mathrm{AGeV}$ regime of the heavy-ion synchrotron SIS18. Equally important is their electromagnetic decay branch into $\mathrm{e}^{+} \mathrm{e}^{-}$pairs. This channel is not subject to strong final state interaction and thus provides an undistorted signal of the matter phase. The goal of the HADES experiments is to measure the spectral properties of the vector mesons such as their in-medium masses and widths.

The HADES heavy-ion program is focused on incident kinetic energies from 1 to $2 \mathrm{AGeV}$. Above about $0.7 \mathrm{AGeV}$ these nucleus-nucleus reactions become increasingly complex as new particles - predominantly mesons - are produced which induce secondary reactions [3]. Some of these elementary reactions are not well known and need to be explored as well. While relativistic heavy-ion collisions produce hadronic matter at a few times normal nuclear matter density and elevated temperature, pion or proton induced reactions embed vector mesons into normal nuclear matter. A dedicated physics program including heavy ions, deuteron, proton and pion beams has been proposed for the HADES detector [4, 5].

Dilepton decays of vector mesons at SIS energies are rare events and their observation presents a challenge for the detector design. Thus, HADES has been equipped with a hadron-blind ring imaging Cherenkov counter, a tracking system and a multiplicity and electron trigger array. A two-stage trigger system selects events containing electron candidates in real time. With its much larger solid angle and improved resolution, HADES continues and has the capability to complete the physics program which was pioneered by the DLS spectrometer at the BEVALAC [6].

\subsection{Detector overview}

HADES features six identical sectors defined by the superconducting coils producing the toroidal geometry magnetic field. The spectrometer has $85 \%$ azimuthal acceptance and covers polar angles between $\theta=18^{\circ}$ and $\theta=85^{\circ}$. The angular and momentum acceptance has been optimized for the detection of dielectron decays of hadrons produced in the SIS energy regime. A section of the detector in the vertical plane containing the beam axis is shown in fig. 1] 


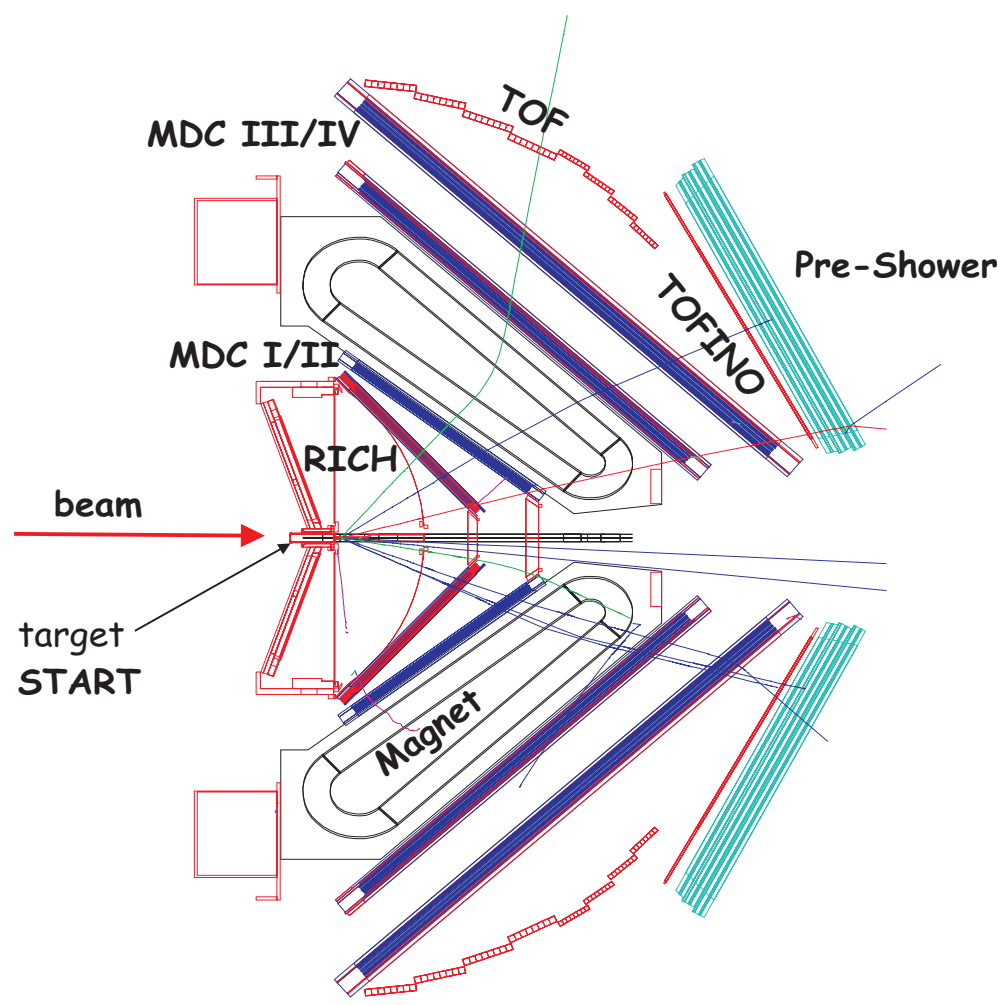

Figure 1: Schematic layout of the HADES detector. A RICH detector with gaseous radiator, carbon fiber mirror and UV photon detector with solid CsI photocathode is used for electron identification. Two sets of Mini-Drift Chambers (MDCs) with 4 modules per sector are placed in front and behind the toroidal magnetic field to measure particle momenta. A time of flight wall (TOF/TOFINO) accompanied by a Pre-Shower detector at forward angles is used for additional electron identification and trigger purposes. The target is placed at half radius off the centre of the mirror. For reaction time measurement, a START detector is located in front of the target. A few particle tracks are depicted too.

Momentum reconstruction is carried out by measuring the deflection angle of the particle trajectories derived from the 4 hit positions in the planes of the Mini-Drift Chambers (MDC) located before and after the magnetic field region. Electron identification is performed with the hadron-blind gas Ring Imaging Cherenkov detector (RICH) together with the Multiplicity and Electron Trigger Array (META) consisting of time-of-flight scintillator walls (TOF/TOFINO) and electromagnetic shower detectors (Pre-Shower). A powerful two-stage trigger system is employed to select events within a predefined charged particle multiplicity interval (first-level trigger LVL1), as well as electron candidates (second-level trigger LVL2).

In the following, a detailed description of the main spectrometer components is given: magnet (sect. 2.1), RICH (sect. 2.2), tracking system (sect. 2.3), META (sects. 2.4 and 2.5) and beam detectors (sect. 2.6). The detector description is 
followed by a discussion of the data acquisition and trigger system (sect. 3). The data analysis framework and the detector performance are discussed in sect. 4.

\section{Major spectrometer components}

\subsection{Magnet}

\subsubsection{Basic design considerations}

The purpose of the magnet is to provide a transverse kick to charged particles in order to obtain their momenta with sufficient resolution being of the order of $\sigma_{p} / p=1.5-2 \%$ for electrons. On the other hand, electron identification with the RICH detector requires a nearly field free region around the target. Furthermore, a large momentum range of $p=0.1-2 \mathrm{GeV} / \mathrm{c}$ should be accepted simultaneously within a large solid angle $\left(\theta=18^{\circ}-85^{\circ}\right.$, as close as possible to full azimuthal coverage). Simulations of reactions in the SIS18 energy regime have shown that these requirements call for a non-focusing spectrometer with a transverse momentum kick $p_{k}$ of about 0.05 to $0.1 \mathrm{GeV} / \mathrm{c}$, where $p_{k}$ is the momentum difference between the incoming and outgoing momentum vectors in the plane perpendicular to the field. The $p_{k}$ is proportional to the product of magnetic field strength $B$ and path length $L$. Assuming a magnetic field path length of $L \simeq 0.4 \mathrm{~m}$, in order to keep the spectrometer compact, the respective magnetic field strength stays below $B=0.9 \mathrm{~T}$.

For such a design, the required momentum resolution can be obtained only by keeping multiple scattering in the region of large magnetic field as small as possible (i.e. allowing no detector material in this region). For high momentum electrons $(p \sim 1 \mathrm{GeV} / \mathrm{c}), p_{k}=0.1 \mathrm{GeV} / \mathrm{c}$ also puts constraints on the position resolution of the particle detectors (MDCs) in front and behind the field region. For example, at $p=1 \mathrm{GeV} / \mathrm{c}$ and $\theta=20^{\circ}$, the deflection angle $\Delta \theta_{k}$ amounts to $5.7^{\circ}$ for $p_{k}=0.1 \mathrm{GeV} / \mathrm{c}$. A simple model calculation assuming two sets of two detectors each spaced by $d=0.3 \mathrm{~m}$ shows that for this case a position resolution of better than $150 \mu \mathrm{m}$ is required to keep the corresponding contribution to the momentum resolution below $1 \%$.

\subsubsection{Field geometry}

The toroidal field geometry provides a field free region around the target and inside the active volume of the RICH. Since the shadow of the coils can be aligned with the detector frames, no additional loss of solid angle is caused by the coils. Although the field strength is rather low, superconducting coils are necessary in order to obtain a compact coil construction. An additional advantage is the low operating cost.

\subsubsection{Superconducting coils}

The system consists of 6 coils surrounding the beam axis. Each coil is separately contained in its individual vacuum chamber. The latter ones are connected to a support ring located upstream of the target. Figure 2 shows a side and a back view of the magnet including the support structure of the coil cases. 
A hexagonal plate, with a hole for the beam pipe, connects the back end of the six coil cases. Through this plate and the support ring, the magnetic forces acting on the coil cases of about $4.9 \cdot 10^{4} \mathrm{~N}$ per coil are compensated. The ring upstream of the target supports the electrical connections between the coils as well as the Helium and Nitrogen cooling lines. No support structure is needed in the region where the drift chambers are located. Furthermore, no material is placed in a $7^{\circ}$ (starting from the target) cone around the beam axis.
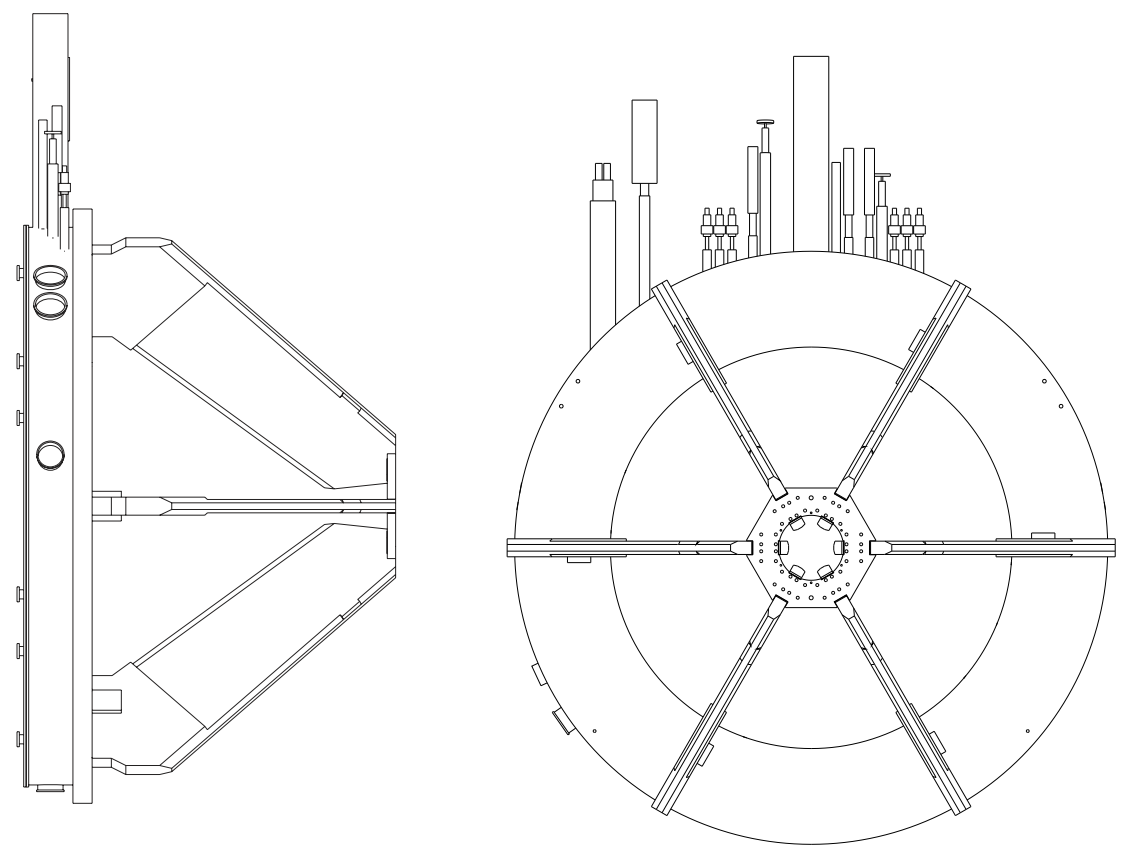

Figure 2: Left: Side view of the superconducting HADES magnet. The outer diameter of the support ring amounts to $3.56 \mathrm{~m}$. Right: Back view

Each coil consists of 2 non-parallel long straight sections connected by two arcs. The magnetomotive force of a coil amounts to 485000 Ampere-turns. Each coil has 140 turns, thus $3464 \mathrm{~A}$ have to be fed through the current leads. The angles of the entrance and exit sections of $40^{\circ}$ and $45^{\circ}$ were chosen to minimize the azimuthal deflection of particles over the whole range of polar angles. Due to the V-shape of the coil a small net focusing - or defocusing, depending on the particle charge - with respect to the azimuthal angles is obtained. The shape and orientation of the coil result in a stronger $p_{k}$ at small polar angles (see table 1). For beam energies of $1-2 \mathrm{AGeV}$, the transverse momentum kick $p_{k}$ provided by the field follows roughly the kinematical variation of the particle momenta with polar angle.

As explained in sect.4.3.1 below, the particle momentum $p$ can be calculated 


\begin{tabular}{|l|l|l|l|l|l|}
\hline$\theta$ & $20^{\circ}$ & $30^{\circ}$ & $40^{\circ}$ & $60^{\circ}$ & $80^{\circ}$ \\
\hline$p_{k 0}[\mathrm{MeV} / \mathrm{c}]$ at $\phi=0^{\circ}$ & 109 & 89 & 73 & 55 & 41 \\
\hline$p_{k 0}[\mathrm{MeV} / \mathrm{c}]$ at $\phi=15^{\circ}$ & 123 & 94 & 76 & 61 & 53 \\
\hline$p_{k 0}[\mathrm{MeV} / \mathrm{c}]$ at $\phi=25^{\circ}$ & - & 99 & 82 & 73 & 85 \\
\hline
\end{tabular}

Table 1: Transverse momentum kick $p_{k 0}$ as a function of the polar $\theta$ and azimuthal $\phi$ angles ( $\phi=0$ corresponds to midplane between adjacent coils) for electrons in the limit of large momenta.

from the relation

$$
p=\frac{1}{2} \frac{p_{k 0}}{\sin \left(\Delta \theta_{k} / 2\right)}+p_{k 1}+2 p_{k 2} \sin \left(\Delta \theta_{k} / 2\right),
$$

where $p_{k 0}$ represents the leading term (see table1). The coefficients $p_{k 1}$ and $p_{k 2}$ are correction terms accounting for the variation of the track length through the field and depend on the sign of the charge. All coefficients depend strongly on $\theta$ and $\phi$.

The field maps exhibited in fig. 3 show the strong inhomogeneity of the field as a function of both polar and azimuthal angles. The maximum field is obtained at the forward arc of the coil and amounts to $3.6 \mathrm{~T}$ at the sector edge $\left(\phi=30^{\circ}\right)$. The field of each sector was mapped using Hall probes and a dedicated optical positioning system. After correcting for the earth magnetic field, the measured field values agree with the ones calculated by TOSCA [7] within better than $1 \%$. This shows that we have a full control of the coil geometry. The agreement with the integrated field (straight line through the field region) is better than $0.2 \%$.
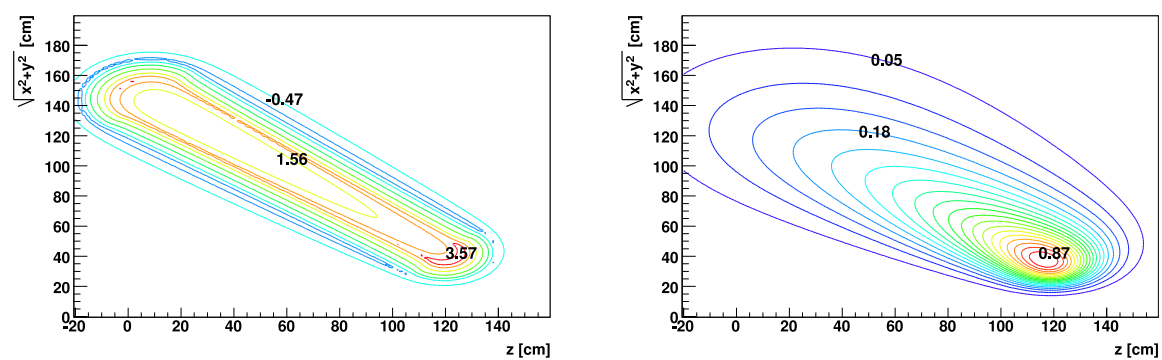

Figure 3: Left: Magnetic field maps, $B$ as function of $\mathrm{z}$ (along beam axis) and $\mathrm{r}$ (per. to the beam axis), at $\phi=30^{\circ}$ (coil position). The step size for the contour lines amounts to $0.24 \mathrm{~T}$. Right: Field map at $0^{\circ}$ (midplane between coils). The step size for the contour lines amounts to $0.046 \mathrm{~T}$.

\subsubsection{Cryo plant}

The coils are surrounded by a liquid Nitrogen cooled shield at $85 \mathrm{~K}$. The flow through this shield amounts to $2 \mathrm{~g} / \mathrm{s}$ including all shielding components. 
The remaining heat load of each coil amounts to $2 \mathrm{~W}$. Together with the heat load on all other components, the total load amounts to $20 \mathrm{~W}$ excluding the current leads. The current leads are cooled with He gas, starting at $4.7 \mathrm{~K}$ and warming up to about $270 \mathrm{~K}$. The heat load depends nearly quadratically on the current with a maximum load of $80 \mathrm{~W}$ (corresponding to $0.7 \mathrm{~g} / \mathrm{s}$ ) at full field. Thus, at full field, the cryo plant (TCF20, [8]) has to provide a cooling power of $100 \mathrm{~W}$, quite close to its $110 \mathrm{~W}$ limit. All heat loads refer to an equivalent cooling power at $4.7 \mathrm{~K}$. In order to avoid gas bubbles inside the thin He pipes cooling the coils, single phase $\mathrm{He}$ at $4.7 \mathrm{~K}$ and $0.29 \mathrm{MPa}$ is used. Above $0.23 \mathrm{MPa}$ (critical point), Helium remains in the gas phase even at low temperatures, with density above the liquid phase at pressures below $0.23 \mathrm{MPa}$. It is afterwards liquefied by expanding to $0.13 \mathrm{MPa}$, providing thermal stability via heat exchangers, connecting thermally the cooling pipes with the liquid reservoir. 


\subsection{The $\mathrm{RICH}$}

\subsubsection{Overview}

The Ring Imaging Cherenkov (RICH) detector constitutes the innermost part of the spectrometer and is designed to identify relativistic $\mathrm{e}^{ \pm}$with momenta $0.1 \mathrm{GeV} / \mathrm{c} \leq p \leq 1.5 \mathrm{GeV} / \mathrm{c}$. The layout, shown in fig. 4. is governed by the limited space between target and tracking detectors and by the need for a low material budget along the particle trajectories to minimize external pair conversion and multiple scattering. The photon detector is placed upstream of the target to spatially decouple the registration of the Cherenkov light from charged particle tracks emitted from the target. The choice of a gaseous photon detector with a photosensitive CSI cathode restricts the sensitivity to the far vacuum ultra violet (VUV) wavelength region.

The radiator gas perfluorobutan $\left(\mathrm{C}_{4} \mathrm{~F}_{10}\right)$ offers high transmission down to $\lambda=145$ nm and a suitable Cherenkov threshold (Lorentz factor $\left.\gamma_{\text {thresh }}=18\right) \quad$ to $\quad$ suppress radiation from muons and hadrons in the given momentum regime. It surrounds the target station in an essentially field free region and is confined by a thin Carbon fiber shell at forward angles (thickness $=0.4 \mathrm{~mm}$ ), by the photon detector $\mathrm{CaF}_{2}$ entrance window and by thin Mylar foils on the beam path. The Cherenkov light is radiated from straight particle trajectories with effective path lengths varying from 36 $\mathrm{cm}$ at $\theta=20^{\circ}$ to $65 \mathrm{~cm} \mathrm{at}$

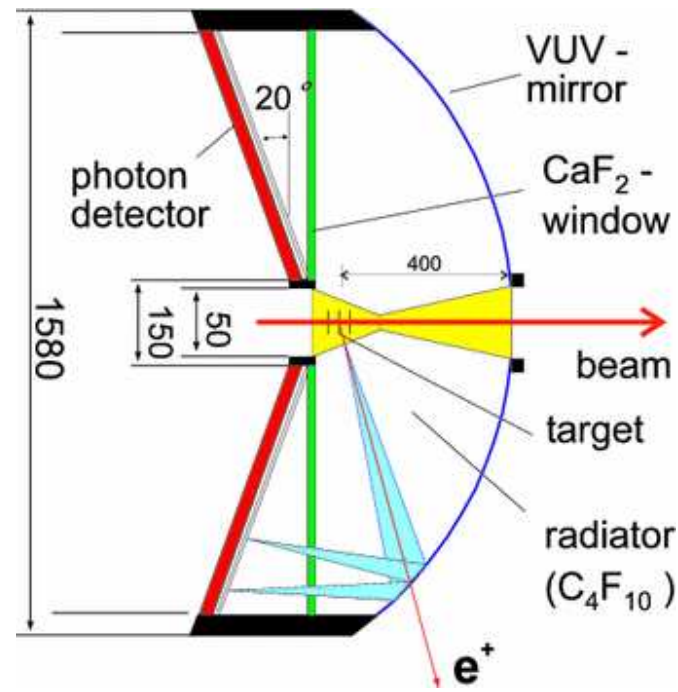

Figure 4: Schematic layout of the RICH, consisting of a Carbon shell mirror, $\mathrm{a} \mathrm{CaF}_{2}$ window and a photon detector. All distances are in millimeter. $\theta=80^{\circ}$.

The photons are reflected by a low mass spherical mirror (curvature radius $R=872 \mathrm{~mm}$ ) onto the photosensitive CsI cathodes of six Multi Wire Proportional Chambers (MWPC) operated with $\mathrm{CH}_{4}$ and equipped with individual pad readout. The optical geometry is chosen such that the photons are focused to rings of almost constant diameter across the whole detector plane. The measured ring center positions are used to disentangle lepton and hadron tracks in high-multiplicity central heavy-ion collisions.

In the following sections we summarize the most important aspects of the photon detector, of the mirror and of the window and present results from 
in-beam measurements. More detailed information on the various RICH components can be found in refs. $9,10,11,12,13$.

\subsubsection{Photon detector}

The RICH photon detector is assembled around the beam pipe from six modules of trapezoidal shape $\left(\right.$ area $\simeq 0.25 \mathrm{~m}^{2}$ ) such that the sensitive planes form a hexagonal pyramid and approximately match the curved focal plane of the mirror. Each module consists of a thin gap $(d=5.5 \mathrm{~mm})$ MWPC with

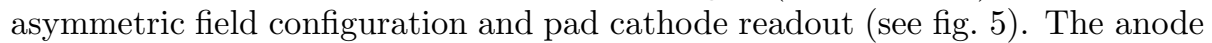
and cathode planes are built from $d_{A}=20 \mu \mathrm{m}$ and $d_{C}=50 \mu \mathrm{m}$ thick Goldplated tungsten wires, respectively. An auxiliary gate (at $\left.U_{G}=+500 \mathrm{~V}\right)$ anode separates the amplification region from freely propagating electrons produced by ionisation processes in the passive gas volume between the MWPC and the $\mathrm{CaF}-2$ entrance window. All wires are oriented in radial direction. The photosensitive cathode plane is segmented into 4712 pads on a Printed Circuit Board (PCB) with Gold-plated conductive layers. The pads are individually coated with Resin Stabilized Graphite (RSG) and a reflective CsI layer [10] acting as photon-electron converter. Operated with pure Methane at atmospheric pressure, the chambers run with anode voltages of 2450-2550 V corresponding to visible gas gains of $(3-9) \cdot 10^{4}$.

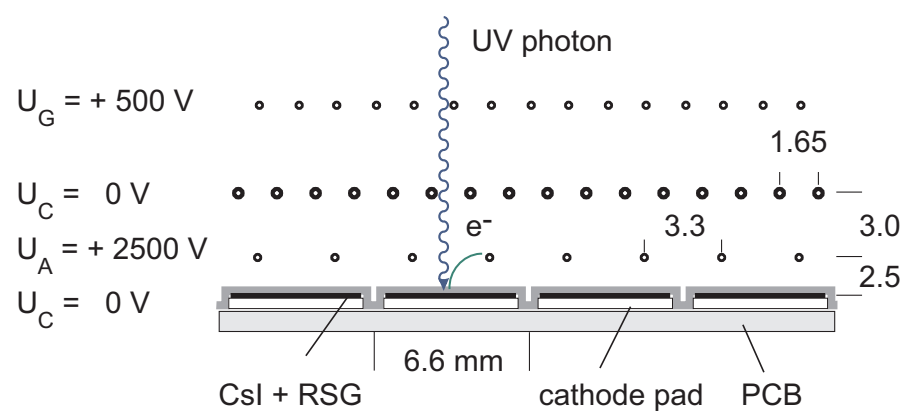

Figure 5: Schematic cross section of the MWPC part of the photon detector. The different wire planes comprise a gate, a cathode and an anode. All distances are given in millimeter. The asymmetric field configuration increases the charge fraction induced onto the pads to about $70 \%$. The entrance window is not shown.

The mismatch of pad plane and mirror focal surface leads to slightly varying image shapes and sizes across the whole sensitive area. With increasing polar angle of $\mathrm{e}^{ \pm}$tracks the Cherenkov images turn from rings to ellipses. To first order, the eccentricity of the ring images is compensated by a variation of the pad size along the radial direction, i.e. the direction of the wires. With a constant pad width of $l_{x}=6.6 \mathrm{~mm}$ perpendicular to the wires, the pad length varies between $l_{y}=7.0 \mathrm{~mm}$ and $l_{y}=4.5 \mathrm{~mm}$ leading to rings of almost constant radius ( $\simeq 3.8$ pads) for all track angles. This facilitates the on-line ring search via simple pattern matrix algorithms implemented in hardware and allows to provide second-level trigger decisions for events with $\mathrm{e}^{ \pm}$tracks [14]. 
The 28272 cathode pads are connected to Preprocessing Front-end Modules (PFM) mounted on the rear side of the cathode PC-board. Each PFM provides 64 charge-integrating amplifiers based on the GASSIPLEX ASIC [15], ADC, zero suppression and event data memory. Using a mixed parallel and daisychained mode, 75 PFMs per detector module are cascaded and connected to two VME-based Readout Controller modules (RC). These provide the interface to the ring processing unit [16] for on-line ring search and the central data acquisition. For details of the RICH readout electronics see also ref. [11].

\subsubsection{VUV mirror, $\mathrm{CaF}_{2}$ window and gases}

A high $\mathrm{e}^{ \pm}$identification efficiency requires a sufficiently large number of detected photons per Cherenkov ring $\left(N_{d e t}^{\gamma} \geq 10\right)$. The short radiator length (i.e. the small number of radiated photons) and the work function of the solar blind CsI photocathode $\left(\lambda_{\max }=220 \mathrm{~nm}\right)$ enforce a spectral sensitivity in the VUV as large as possible. With the lower wavelength limit given by the transmission cutoffs of the gases $\left(\lambda_{\min } \simeq 145 \mathrm{~nm}\right)$, this requirement can only be met by a mirror substrate of very low surface roughness with a $\mathrm{MgF}_{2}$ protected aluminum coating and $\mathrm{a} \mathrm{CaF}_{2}$ entrance window for the photon detector.

The mirror design goal was a low mass substrate with a thickness $d$ such as $d / X_{0} \leq 1 \%$, comparable to the ones of the target and radiator materials. Simultaneously, the surface and optical imaging quality should guarantee for almost uniform rings with minimum distortions of ring shapes across the whole detector plane. This would allow both on-line $\mathrm{e}^{ \pm}$recognition with a single ring finding algorithm implemented in hardware and the extraction of hit points from high resolution ring center analysis to be used as an additional information for lepton tracking. These requirements lead to a spherical mirror with the properties as listed in table 2 .

\begin{tabular}{|l|r|}
\hline Parameter & Value \\
\hline Outer diameter $D$ & $1440 \mathrm{~mm}$ \\
Radius of curvature $R_{i}$ & $872 \mathrm{~mm}$ \\
Substrate thickness $d$ & $\leq 2 \mathrm{~mm}$ \\
Radiation length $X_{0}$ & $\geq 20 \mathrm{~cm}$ \\
Reflectivity $R(\lambda=150 \mathrm{~nm})$ & $\geq 70 \%$ \\
Surface roughness $\sigma(\mathrm{rms})$ & $\leq 3 \mathrm{~nm}$ \\
Surface slope error $\mathrm{SSE}_{80}$ & $\leq 1 \mathrm{mrad}$ \\
Areal density $\rho d$ & $<0.3 \mathrm{~g} / \mathrm{cm}^{2}$ \\
\hline
\end{tabular}

Table 2: Geometrical and optical parameters of the HADES RICH mirror.

Since a self-supporting mirror shell made from a single piece was ruled out, we have developed and constructed, together with DSS1, a mirror with

\footnotetext{
${ }^{1}$ DSS Dornier Sat. Syst., D-81663 München, Germany.
} 

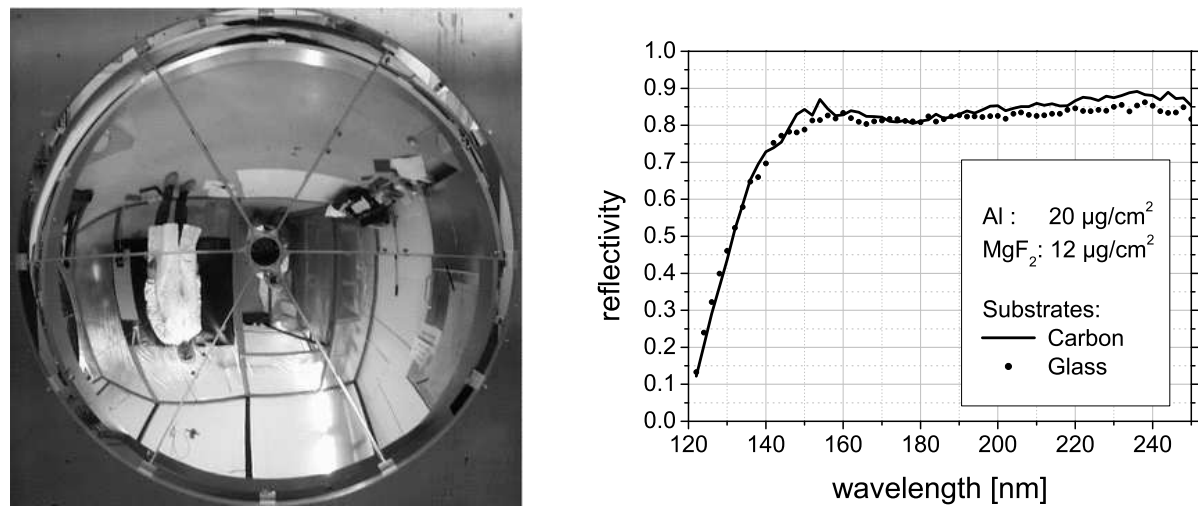

Figure 6: Left: Photo of the RICH mirror. Right: Reflectivities of glassy Carbon samples in comparison to float glass samples.

a segmented substrate shell and a six-fold radial support structure with spokes matched to the coil cases of the superconducting magnet. As substrate material we have chosen glassy Carbon, an isotropic and homogeneous material produced by pyrolytic conversion of Phenol type resins [17] and commercially available as Sigradur ${ }^{\circledR}$ from the company HTW2. After production, the eighteen substrate panels ( 3 for each sector) have been individually grinded to $2 \mathrm{~mm}$ thickness, polished to the required surface roughness and finally machined to the desired shape. The panels were coated with a $20 \mu \mathrm{g} / \mathrm{cm}^{2}$ Aluminium reflective layer followed by a $12 \mu \mathrm{g} / \mathrm{cm}^{2} \mathrm{MgF}_{2}$ protection layer. The deposition was performed in the Ultra-High-Vacuum box coater [18] installed at Technische Universität München following the procedure described in [19].

The optical quality of the panel surface was measured in terms of surface slope errors $\mathrm{SSE}_{80}$ and was found to change from typically $0.5 \mathrm{mrad}$ to 0.8 mrad. The surface roughness and the achievable VUV reflectivity were evaluated with various methods from witness samples obtained during the panel cutting process. Both, the visible surface roughness and the VUV reflectivity show good agreement with those from float glass samples and are consistent with the assumption of a micro roughness of $\sigma \simeq 2-3 \mathrm{~nm}$. Measured reflectivities are shown in the right panel of fig. [6 and exhibit constant values around $R=80 \%$ down to $150 \mathrm{~nm}$. Figure 6 shows the mirror after assembly under clean room conditions and prior to integration into the RICH system. For details of the mirror design, material parameters, production techniques, and optical quality mesurements the reader is referred to ref. [12].

The $\mathrm{CaF}_{2}$ window separates the gas volumes of radiator and photon detector. It was assembled from altogether 64 hexagonally shaped single crystal $3(200 \mathrm{~mm}$ in diameter, $5 \mathrm{~mm}$ thick each). A high VUV transmission was achieved through

\footnotetext{
${ }^{2}$ HTW GmbH, D-86672 Thierhaupten, Germany.

${ }^{3}$ Korth Kristalle, D-24161 Altenholz.
} 

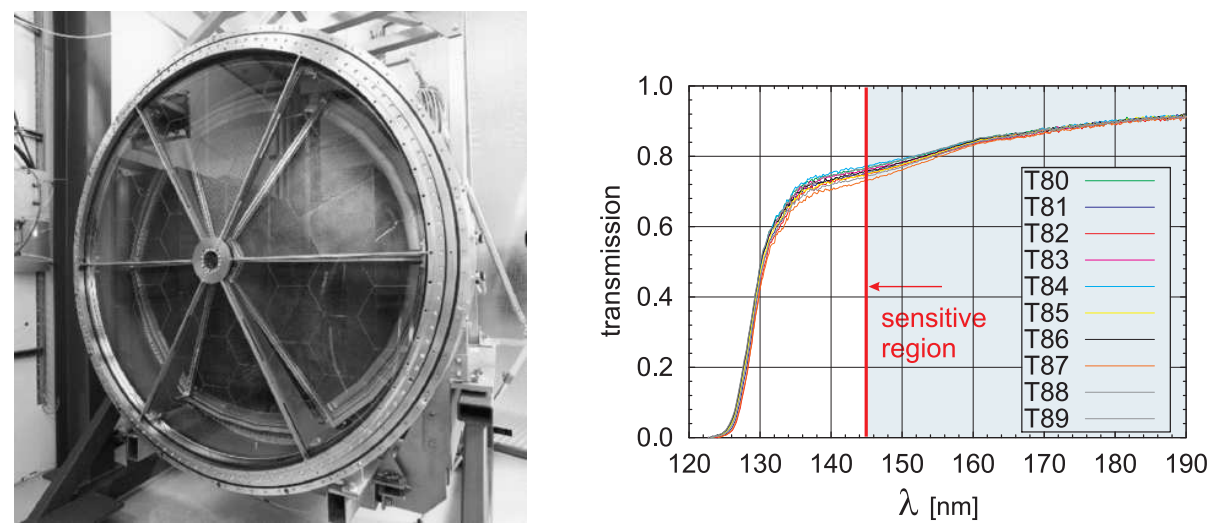

Figure 7: Left: View of the photon detector with the assembled $\mathrm{CaF}_{2}$ entrance window. Right Transmissions of individual $\mathrm{CaF}_{2}$ crystals $\mathrm{T} 80$ to T89.

proper selection of the $\mathrm{CaF}_{2}$ raw material followed by an optimized polishing technique and was verified for each crystal individually (fig. 7). The polished crystals were glued together $(\simeq 100 \mu \mathrm{m} 3 \mathrm{M}$ Scotch DP190) to one single disk of $1500 \mathrm{~mm}$ diameter (see fig. 17) with the central hexagon containing a hole for the beam tube. Installed in vertical position, the disk is connected to stainless steel mounting frames through a $2 \mathrm{~mm}$ thick and $10 \mathrm{~mm}$ wide Viton ${ }^{\circledR}$ interface to damp the influence of the different thermal expansion coefficients and to minimize mechanical stress effects due to gravitational forces. Six thin spokes support the window on both sides against pressure differences.

The gas volumes of radiator and photon detector amount to about 700 liters each and are filled to $\simeq 10-40 \mathrm{hPa}$ above ambient pressure. The photon detector is supplied from commercially available bottles with Methane $\left(\mathrm{CH}_{4}\right.$, purity 4.5$)$ through an open system at a typical gas flow of $300-350 \mathrm{l} / \mathrm{h}$. For the radiator, a batch of prepurified radiator gas $\mathrm{C}_{4} \mathrm{~F}_{10}$ (3M CEA410) is stored in a liquid reservoir and recirculated in a closed system via an evaporation and compression-liquefaction chain. The observed gas losses amount to about $1.5-2 \mathrm{l} / \mathrm{h}$ at a standard flow of $300 \mathrm{l} / \mathrm{h}$. Absolute pressure and gas flows of both systems are steered and monitored by a PLC-controlled gas supply system [13] which also keeps the pressure difference $\Delta P$ between radiator and MWPC below $3 \mathrm{hPa}$ to protect the fragile $\mathrm{CaF}_{2}$ window. The purity of the gases is monitored on the inlet and outlet side through frequent VUV transmission measurements utilizing a D2 light source combined with a standard monochromator grid-photodetector setup. The achieved gas transmissions are compiled in fig. 8 together with the ones for $\mathrm{CaF}_{2}$, fused quartz, and the CsI quantum efficiency.

\subsubsection{Performance and results from in-beam measurements}

The performance of the RICH is governed by the average number of photons detected for each $\mathrm{e}^{ \pm}$induced ring. The number of detected photons per ring is 


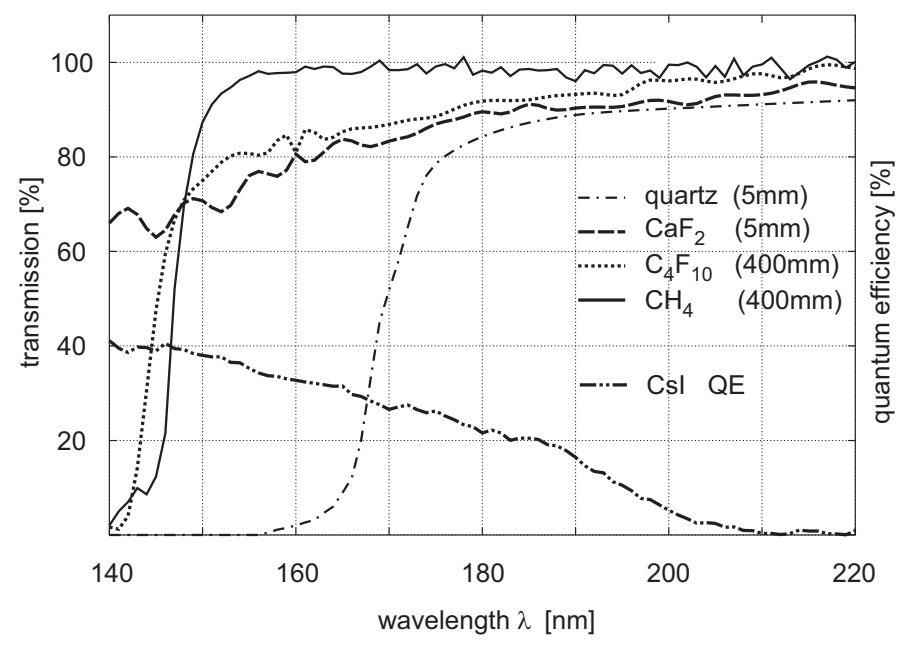

Figure 8: Measured transmissions of radiator, detector gases, $\mathrm{CaF}_{2}$ window and quartz (for comparison) are plotted (left abscissa) together with the the CsI quantum efficiency (right abscissa).

$N_{d e t}^{\gamma} \approx N_{0} Z^{2} D \overline{\sin ^{2} \Theta_{C}(\lambda)}$. For relativistic $\mathrm{e}^{ \pm}\left(Z= \pm 1, \beta \simeq 1, \Theta_{C} \simeq 3.15^{\circ}\right.$ is the Cherenkov angle) it depends on both the available radiator length $D$ and a figure of merit $N_{0}=k \int_{E_{1}}^{E_{2}} \epsilon\left(E_{\gamma}\right) \cdot d E_{\gamma}$, where $k=379 \mathrm{eV}^{-1} \mathrm{~cm}^{-1}$ and $\epsilon\left(E_{\gamma}\right)$ is the global detection efficiency including the optical and electrical properties of the system. The optical transmissions (fig. 8) and the parameter values measured for small samples translate to an optimum $\mathrm{N}_{0} \approx 109$ corresponding to $10<N_{\text {det }}^{\gamma}<18$.

Simulations have shown that for values $N_{\text {det }}^{\gamma}<9$ the efficiency for $\mathrm{e}^{ \pm}$identification and hence the on-line trigger efficiency are significantly affected. To check the achieved performance of the fully assembled system we have measured $N_{0}$ for all six sectors with a dedicated ion beam setup. As a "calibrated" VUV light source we have used the known amount of Cherenkov photons radiated from single relativistic Carbon ions $(E=0.6 \mathrm{AGeV}, \beta=0.794)$ when passing through $\mathrm{SiO}_{2}$ and $\mathrm{MgF}_{2}$ crystals placed close to the normal target position. The radiator thickness and hence the light output was chosen such that individual photons could be spatially resolved on the cathode pad plane. The method is described in detail in ref. [20]. The measured pulse height spectra and angular distributions of the photons were used to quantitatively model the electronic detector response needed for the general HADES detector simulation package. From the analysis of the single photon induced pad clusters the aforementioned gas amplifications of about $(3-9) \cdot 10^{4}$ and a single electron detection efficiency $\epsilon \simeq 95 \%$ were deduced. Counting the number of registered photons leads to 
experimental figure of merit values $80<N_{0}<100$ varying slightly from sector to sector. The measured average light yield is about $10-20 \%$ lower than expected from the small sample laboratory measurements. We cannot identify a well defined reason for this discrepancy and rather attribute it to deficiencies and inhomogeneities across the whole detector area for all involved components and parameters.
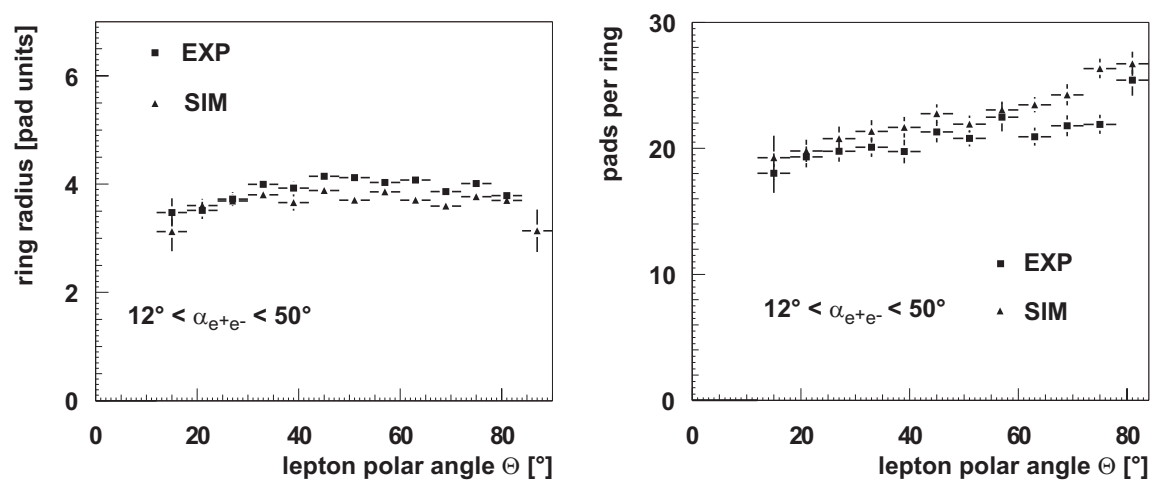

Figure 9: Polar angle distributions of Cherenkov ring properties for single electrons from open $\mathrm{e}^{+} \mathrm{e}^{-}$- pairs produced in the reaction $\mathrm{C}+\mathrm{C}$ at $1 \mathrm{AGeV}$ (exp). Simulation results (sim) are shown for comparison. The error bars reflect the widths of the nearly Gaussian multiplicity distributions at each angle. Left: Ring radius vs lepton polar angle Right: Mean number of pads within a region of $13 \times 13$ pads around ring centers.

To verify the obtained results we have analyzed the detector response also for relativistic electrons and positrons emitted as open pairs (opening angle $\alpha_{e^{+} e^{-}}>12^{\circ}$ ) in fully reconstructed $\pi^{0}$ Dalitz decays from $\mathrm{C}+\mathrm{C}$ collisions at 1 $\mathrm{AGeV}$ beam energy. These dielectrons are emitted from the target and hence exhaust the full radiator length, in contrast to the dominant background of $\mathrm{e}^{ \pm}$ candidates from external pair conversion. In fig. 9 we have plotted the measured ring radius and the average number of fired pads within a region of $13 \times 13$ pads around the ring center for various polar angles of the emitted electrons. The data have been obtained by averaging over the full azimuthal detector acceptance, i.e. all six MWPC modules, and are characterized by nearly Gaussian multiplicity distributions at each polar angle. The small variation of ring radius nicely reflects the adjusted pad design of the cathode plane (see sect. 2.2.2). The continuous rise of pad multiplicity with polar angle is a consequence of the increasing radiator length, from about $36 \mathrm{~cm}$ to $65 \mathrm{~cm}$. Averaged over all 6 sectors, one observes experimentally a mean of $19.3 \pm 2.8$ pads around $\theta=25^{\circ}$ and $22.3 \pm 2.0$ pads around $\theta=75^{\circ}$. The large widths partly reflect variations of optical and electrical performance of the different detector modules. Comparing the experimental values with those from simulations based on the experimental $N_{0}$ values yields a reasonable agreement. The differences at larger angles point to possible losses in radiator transmission at longer path lengths and/or to local imperfections in photocathode quantum efficiency or mirror reflectivity. 
In summary, the overall performance of the RICH detector system, although slightly lower than in the original design, has proven to be sufficient for $\mathrm{e}^{ \pm}$ identification in nuclear collisions (see sect. 4.5). 


\subsection{Tracking system}

\subsubsection{Overview}

The high-resolution spectroscopy of vector mesons $\left(\sigma_{M_{e^{+} e^{-}}} / M_{e^{+} e^{-}} \simeq 2.5 \%\right)$ in heavy-ion collisions via their dielectron decay channel defines the decisive design and performance constraints on the HADES tracking system. To reach this goal, an intrinsic spatial cell resolution of the order or better than $150 \mu \mathrm{m}$ along with the reduction of multiple scattering in detector materials and air, high efficiency and a large acceptance are crucial requirements for the success of the experimental program. Extended design studies and prototyping 21, 22, 23 preceded the production of the 24 Mini Drift Chambers (MDCs) of four different sizes, conducted by GSI Darmstadt, LHE/JINR Dubna, FZ DresdenRossendorf [24], IPN Orsay and University of Frankfurt.

The HADES tracking system consists of 24 trapezoidal planar MDCs symmetrically arranged in six identical sectors. It provides a polar angle coverage between $18^{0}$ and $85^{\circ}$ around the beam axis, forming four tracking planes (I-IV) of increasing size. In each sector, two modules (planes I and II) are located in front of and two (planes III and IV) behind the toroidal magnetic field of the superconducting magnet, as shown in fig. 10. The region between the six coils of the magnet dictates the active area of the chambers.

During the construction special emphasis was put on the use of low-mass materials for window foils (aluminized Mylar), wires (bare aluminum) and counting gas (Helium-based) in order to minimize multiple scattering. The total detector thickness per chamber is about $5 \cdot 10^{-4}$ in units of radiation length, giving a total close to $0.2 \%$, whereas the air in the tracking system represents $0.3 \%$. These values allow to achieve the momentum resolution needed to accomplish the physics demands as will be shown in sect. 4.3.4.

The technical features are summarized in sect. 2.3.2. Section 2.3 .3 briefly sketches the aspects concerning the alignment of the different modules with respect to each other. In sect. 2.3.4 the performance parameters of the drift chambers regarding detection efficiency, track reconstruction efficiency and spatial resolution are discussed. Section 4.3 .4 presents the corresponding achieved momentum resolution.

\subsubsection{Detector characteristics}

To cope with ambiguities in track reconstruction in the high multiplicity environment of a heavy-ion reaction (for central $\mathrm{Au}+\mathrm{Au}$ collisions at $1 \mathrm{AGeV}$ incident energy a maximum cell occupancy of $30 \%$ is estimated), all chambers are composed of six sense/field wire layers oriented in six different stereo angles, i.e. $\pm 0^{0}, \pm 20^{0}, \pm 40^{0}$, see fig. 10. This favors maximum spatial resolution in polar direction, which points in the direction of the momentum kick. All four chamber types contain about 1100 drift cells each, with increasing size from $5 \times 5 \mathrm{~mm}^{2}$ (plane I) to $14 \times 10 \mathrm{~mm}^{2}$ (plane IV). The chambers provide active areas from $0.35 \mathrm{~m}^{2}$ up to $3.2 \mathrm{~m}^{2}$, thus covering the same solid angle per sector. The main feature of the design and the operation parameters of the chambers is the consequent implementation of the low-mass concept, as already pointed 

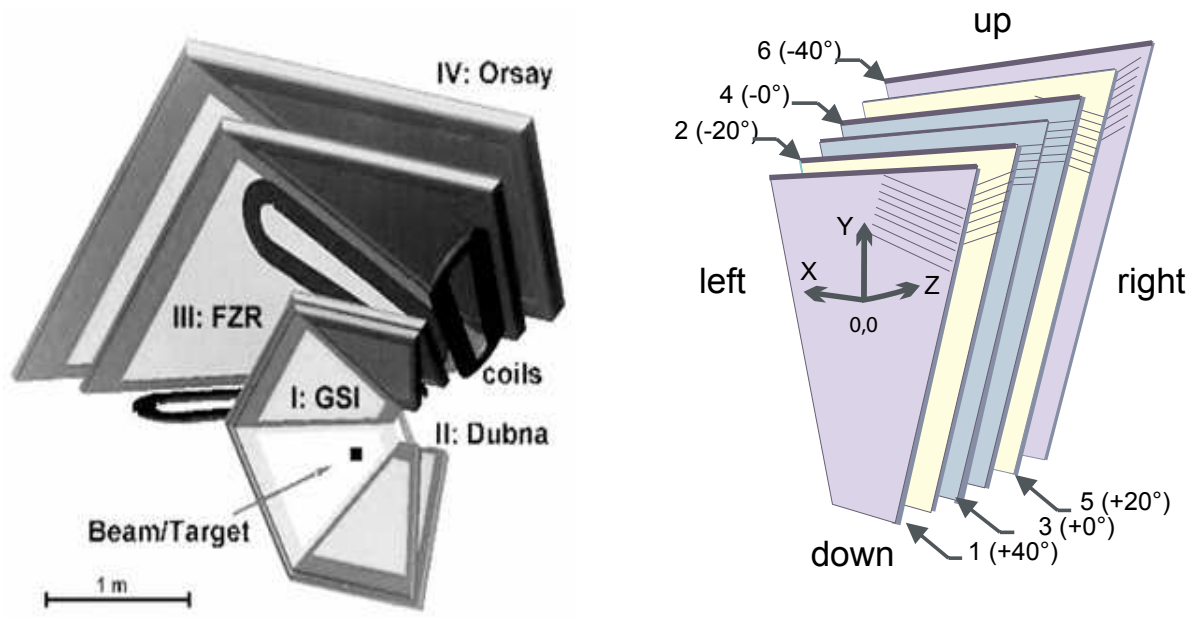

Figure 10: Left: Schematic layout of the HADES tracking system. Two sets of Mini Drift Chambers (MDCs) with 24 wire planes per sector are placed in front and behind the magnetic field to measure particle momenta. Right: Schematic view of the six anode wire frames inside a HADES MDC, representing the six stereo angles.

out above. Resulting from the extensive R\&D phase [21] these requirements are met by three preventive measures: (i) cathode and field wires made of annealed aluminum (planes I-III: bare, IV: Gold-plated) with $80 \mu \mathrm{m}$ and $100 \mu \mathrm{m}$ diameter spanned with tensions between 80 and $120 \mathrm{cN}$, depending on the chamber type, (ii) a Helium-based counting gas (Helium:Isobutane $=60: 40)$ and (iii) entrance windows made of $12 \mu \mathrm{m}$ aluminized Mylar.

The $20 \mu \mathrm{m}$ (planes I- III) and $30 \mu \mathrm{m}$ (plane IV) thick Gold-plated Tungsten sense wires are strained with an initial tension of 40 and $110 \mathrm{cN}$, respectively. To compensate for the total wire tension after being released from the assembly table, chamber frames of planes I- III have been pre-stressed before wire gluing. Together with the requirements concerning the acceptance this resulted in a sophisticated layer frame design with only $3 \mathrm{~cm}$ width, given by the coil case shadows, for the inner-most chambers. The chambers of the outer-most plane IV, which did not use the pre-stressed technique employ two extra Carbon bars to keep the wire tension loss due to deformation below $10 \%$.

The experiments with HADES are expected to run at least ten years. Creeping of the Aluminum wires and ageing are the main concerns with respect to the long-term stability of the chambers. Creeping has been systematically investigated in tension loss test series, yielding a $10 \%$ loss in tension within five years. This has been confirmed by remeasurements in one chamber of plane III [24]. Ageing is mainly caused by the accumulated dose in combination with the materials used for construction and operating the chambers. For example, Epoxy from Araldite ${ }^{\circledR}$ is used for gluing the wires on the frames from Stesalit. The gas system is running in a re-flow mode with typically $10-20 \%$ fresh gas and 
continuous purification employing two large volume Copper catalyzer-filled cartridges, which keep the Oxygen contamination level below $15 \mathrm{ppm}$. Two drift velocity monitors 25] provide a sensitive control of the gas quality by measuring the drift velocity with a precision of better than $0.2 \%$. In addition, the simultaneous monitoring of the relative gains allows conclusions on the gas contamination, e.g. due to Oxygen. The expected maximum charge is of the order of $10 \mathrm{mC}$ per year and centimeter of sense wire. An accelerated ageing test with ${ }^{55} \mathrm{Fe}$ using two prototype chambers exhibited no noticeable gain drop $(<5 \%)$ for a time period of two years of continuous running [21].

These results on creeping and ageing, together with the careful selection of materials and running conditions, suggest that the requirements for the projected long-term operation of the HADES tracking system can be met.

The drift chamber signals are read-out and digitized by means of dedicated, customized boards mounted on the chamber frames, not extending into the active area. Hence, special emphasis was put on the integration of the modular front-end electronics, realized with analog boards (16 channels) mounted on digitization boards (64 or 96 channels). Four sense wires are connected by flexible printed circuits to the analog boards [26], housing ASD8-B chips [27] (8 channels, $1 \mathrm{fC}$ intrinsic noise, $30 \mathrm{~mW} / \mathrm{ch}$, adjustable threshold) for differential amplification, shaping and discrimination. These chips deliver logical signals with variable width, being equivalent to the time the shaped signal exceeds the given threshold. The logical signals are fed to TDC chips (CMOS, 8 channels/chip, $0.5 \mathrm{~ns} / \mathrm{ch}$, common-stop, $1 \mu$ s full range) on the digitization boards. This semi-customized ASIC is multi-hit capable and thus allows to detect also the Time over Threshold (ToT) of each hit. Besides spike and zero suppression this chip offers the possibility of internal calibration, activated by a separate trigger type. The design of the front-end electronics was decisively influenced by minimizing the noise level on-line. In addition, the ToT information is a valuable tool to discriminate remaining noise hits off-line.

\subsubsection{Alignment}

The aimed-at performance of the HADES detector can only be achieved with a very precise knowledge of the positions of all detector components, and in particular of the MDC tracking chambers. As discussed above, geometric surveys can only provide part of the necessary information, and additional correction parameters must be obtained from the event data themselves. Presently we have developed alignment procedures based on (i) straight-track reconstruction of data taken without magnetic field, (ii) straight tracks from cosmic radiation, and (iii) kinematically constrained events from proton-proton elastic scattering. For all of those, iterative fitting algorithms allow to produce a set of translation and rotation parameters for each of the 24 tracking chambers, as well as for the other position-sensitive detector parts (TOF wall modules, Pre-Shower chambers, and RICH). Using straight tracks selected from a well defined experimental situation (beam energy, target, trigger), a global alignment can be obtained and the corresponding parameters entered in the database. Although the precision with which the parameters were determined is of the order or bet- 
ter than $0.1 \mathrm{~mm}$, they represent only a global alignment, possibly biased by the events topology, which has to be further refined by tuning individual layer or wire groups offset parameters for individual chambers. This is still an on-going work. These parameters enter the geometry used in the track reconstruction.

\subsubsection{Tracking performances}

Detection efficiency. The detection efficiency of the wire layers has been investigated by measurements using $\beta$ rays from ${ }^{90} \mathrm{Sr}$ sources, cosmic rays and reconstructed tracks from physics runs. Consistent detection efficiencies for minimum ionizing particles detected in the two inner drift chamber planes of about $90 \%$ and $97 \%$ were obtained, whereas the layer efficiency of the outer drift chambers reaches almost $100 \%$. Due to the small drift cell size of the inner drift chambers, the efficiency depends strongly on the applied high voltage and the read-out threshold. The lower efficiency of inner drift chambers results from operation at a smaller high voltage of $-1750 \mathrm{~V}$, respectively $-1800 \mathrm{~V}$ as compared to the prototype in-beam test at typically $-2000 \mathrm{~V}$. The operation points of the experimental runs have been chosen for stable performance during data taking.

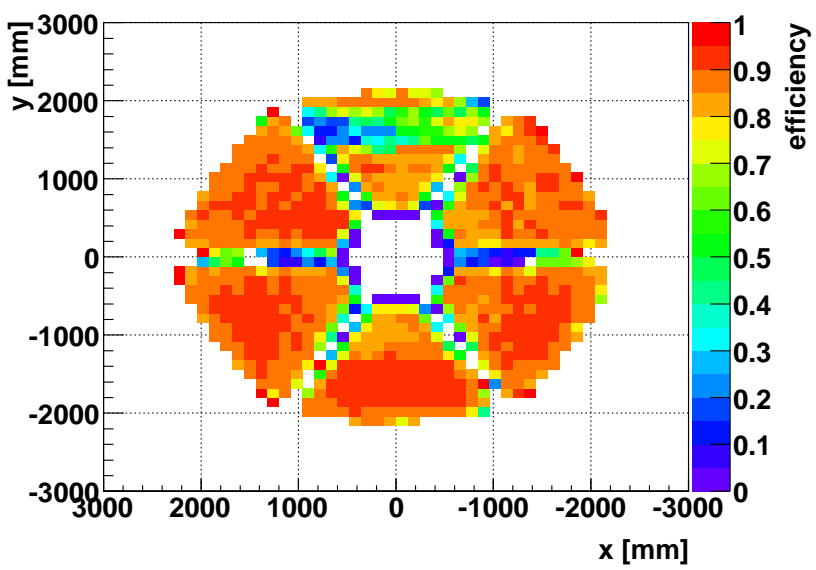

Figure 11: Reconstruction efficiency in the HADES acceptance for protons from $\mathrm{p}+\mathrm{p}$ elastic collisions at $1.25 \mathrm{GeV}$ kinetic energy. Regions of reduced efficiency in the upper sector are due to read-out failures and are fully taken into account through our GEANT simulation.

Track reconstruction efficiency. The track reconstruction efficiency has been investigated in proton-proton elastic scattering at $1.25 \mathrm{GeV}$ kinetic energy which allows to prepare a clean track sample using angular (theta and phi) correlations of hits in the TOF and Pre-Shower detectors only. Knowing that each detected hit in the outer detector is caused by a p + p elastic scattering event, one can obtain the reconstruction efficiency from the inner and outer tracking system. Protons from elastic scattering are reconstructed by the candidate 
search (see sect. 4.2.1) with an efficiency close to $100 \%$. Requiring a fitted inner MDC segment (see sect. 4.2.2) reduces the efficiency to about $92 \%$, a fit with both inner and outer MDC segments to $87 \%$. A full track reconstruction, including efficiency of the momentum determination via Runge-Kutta method, (see sect. 4.3.3) results in an efficiency of $86 \%$. Figure 11 displays the reconstruction efficiency projected on a plane perpendicular to the beam axis. The reconstruction inefficiency visible in the upper sector is caused by readout electronics temporarily failing during this run. The corresponding analysis and correction factors were tuned accordingly and controlled against the elastic scattering angular distribution.
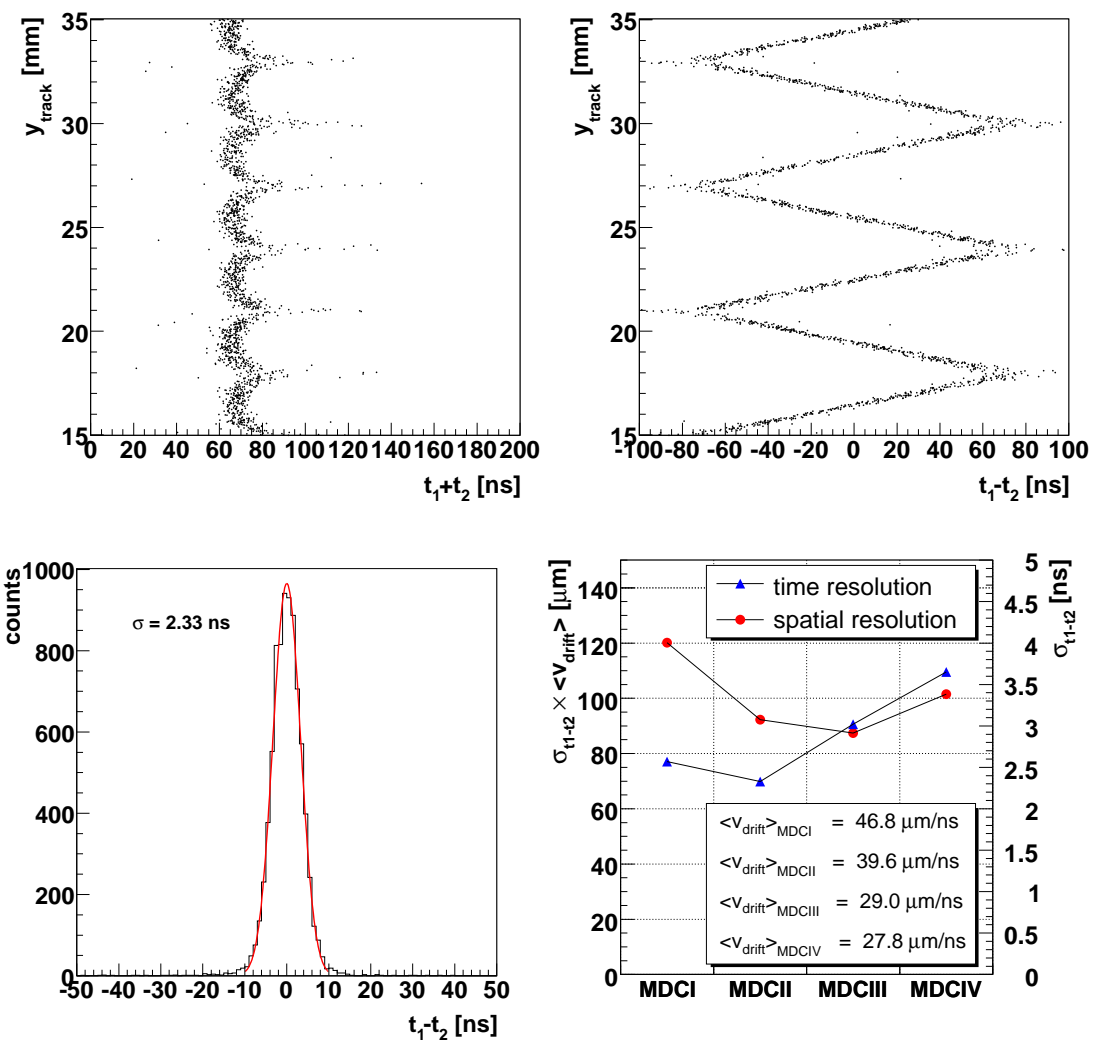

Figure 12: Top left: Sum of the measured drift times of two adjacent drift cells of the 0degree layers versus the y-position of the reconstructed trajectory in the MDC coordinate system. Top right: Difference of the measured drift times of two adjacent drift cells. Bottom left: Projection, after transformation, of the measured drift time differences, shown here for MDCII. Bottom right: Time resolution (blue triangle) and corresponding spatial resolution (red dots) for the different drift chamber types MDCI-IV. The spatial resolution has been obtained by multiplying the drift time resolution by the average drift velocity. 
Spatial resolution. First resolution measurements with a prototype of a plane II type chamber using a $2.1 \mathrm{GeV} / \mathrm{c}$ proton beam and an external silicon tracker achieved a spatial resolution $\sigma=70 \mu \mathrm{m}$ over $70-80 \%$ of the drift cell [22]. These measurements were performed at an optimum high voltage setting of $-2000 \mathrm{~V}$. The spatial resolution of the drift cells under in-beam conditions have been investigated with the so-called self-tracking method.

This method exploits time measurements of the same track in two adjacent drift cells, one in each of the two 0-degree layers (see fig. 10). For a given impact angle of the particles on the chamber, the sum of both drift time measurements is constant over a large fraction of the cell. Once the distance between the signal wires is known, the distance-time correlation, the drift velocity and the time resolution can be obtained. The drift velocity and distance-time correlation have been found to be in good agreement with GARFIELD simulations of the drift cells 28]. Typical values of the difference between the simulated value and the measured one stay below the simulated time dispersion of $2 \mathrm{~ns}$. The latter simulations were then used to provide the distance-time correlation for the track fitting.

Figure 12 shows the results of the method for the MDCII case. Particle tracks (mostly from protons and pions in the region of minimum ionisation) close to perpendicular impact from a proton-proton experiment at $3.5 \mathrm{GeV}$ kinetic beam energy were selected. The sum of the two measured drift times (fig. 12 top left) shows nicely that the sum is almost constant (deviations occurs only at the wire positions), but it suffers from trigger time fluctuations. The time difference (fig. 12 top right) shows a much cleaner correlation pattern, which is not affected by this event-to-event fluctuation of the trigger. By fitting the lines of this z-shaped pattern and applying an appropriate transformation, a straight pattern is obtained, which is further projected. Assuming equal contribution of both drift cells, time resolutions are obtained (fig. 12 bottom left), ranging from $2.3 \mathrm{~ns}$ up to $3.6 \mathrm{~ns}$, slightly depending on chamber gas and cell geometry. Corresponding spatial resolutions were deduced for all MDC using averaged drift velocities (fig. 12 bottom right). They range from 70 up to $110 \mathrm{~mm}$.

Energy loss measurement. Due to the lack of ADCs there is no direct measurement of the deposited charge in the MDC cells. From the width of the drift time signal (Time over Threshold (ToT)) an unambiguous measure of the energy loss of the particle can be extracted. The measured ToT depends on the gas mixture, on the reduced electric field $E / P$, on the track geometry, on the drift cell size and on the threshold setting of the ASD8 chip. Special attention was paid to the unfolding of these dependencies to allow averaging over all drift cell measurements contributing to a particle track.

For the following discussion the particle tracks are parameterized via minimum distance of the track to the sense wire and the impact angle with respect to the wire plane. The measured ToT depends on the distance from the sense wire, the impact angle (fig. 13 left) and the momentum of the particle (shown for selected protons in fig. 13 right).

To calibrate the measured ToT of the single drift cells the correlation of the 

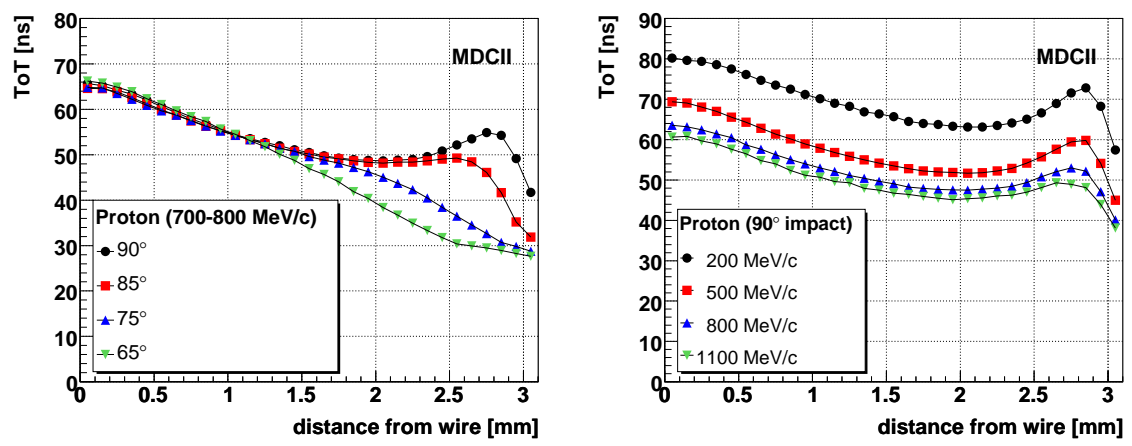

Figure 13: Left: Dependence of ToT on the distance from wire to particle track for 0.7-0.8 $\mathrm{GeV} / \mathrm{c}$ protons at 4 impact angles. Right: Same for protons with a average impact angle of $90^{\circ}$ and different momenta (shown for MDCII).

ToT with the particle energy loss is fitted by the function

$$
T o T=f(d E / d x)=c_{0}+c_{1}\left[\log _{10}\left(d E / d x+c_{3}\right)\right]^{c_{2}},
$$

with the parameters $c_{0}, c_{1}, c_{2}$ and $c_{3}$. Knowing the value of the momentum and the particle type from the other detectors, the energy loss of the particle can then be calculated using the Bethe-Bloch formula. The procedure is performed

Figure 14: ToT versus energy loss correlation for protons at different angles of incidence. The distributions have been fitted by the function (2).

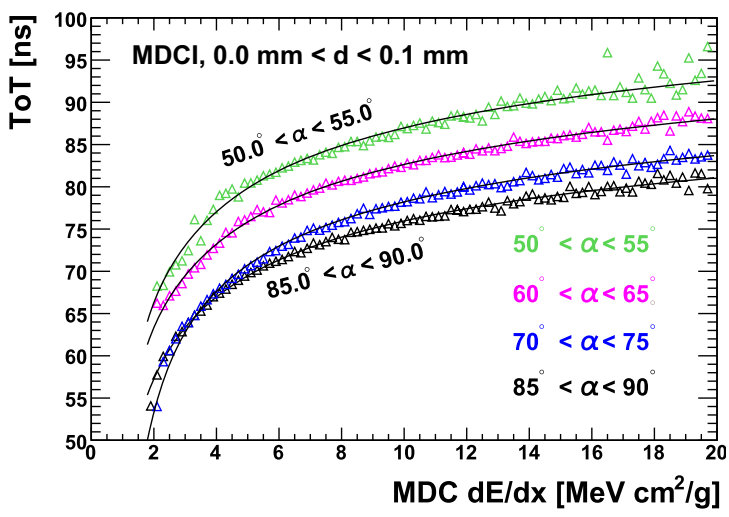

for intervals of $5^{\circ}$ impact angle and $100 \mu \mathrm{m}$ distance from the sense wire for all 4 MDC types (fig. 14).

The correlation between the measured ToT and the energy loss of the particles track is non-linear. This compression of the correlation is most pronounced for strongly ionizing particles. The shape of the correlation curve depends on the shape of the signal and thus on the arrival time of the drifting electrons on the sense wire and also on the signal shaping performed in the readout electronics. To allow for averaging over all drift cell measurements, all contributing measurements are normalized to one reference measurement employing the inverse 
correlation function (2). Finally, a truncated mean method has been applied to get a better resolution. After truncation, on average 18 cells out of 24 remain, corresponding to a $20 \%$ cut.

Figure 15: Correlation of energy loss and polarity times momentum of particle tracks. The energy loss is averaged over all contributing drift cells of a track through the 4 MDC planes and a truncated mean method has been applied. The solid lines represent the energy loss calculated from the Bethe-Bloch formula.

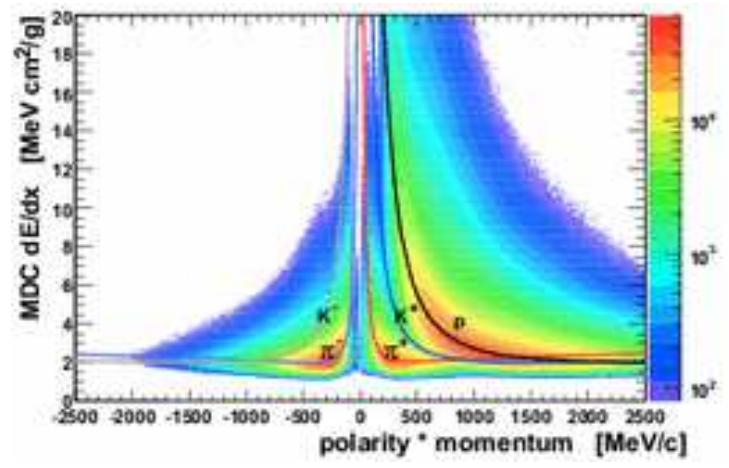

Figure 15 shows the correlation of the calibrated and truncated energy loss with particle momentum. The calculated energy losses from the Bethe-Bloch formula are represented as solid lines. Up to a momentum of about $0.7 \mathrm{GeV} / \mathrm{c}$ the MDC energy loss measurement can be exploited to separate $\pi^{+}$from protons or to improve the signal to background ratio in a $\mathrm{K}^{+}$measurement.
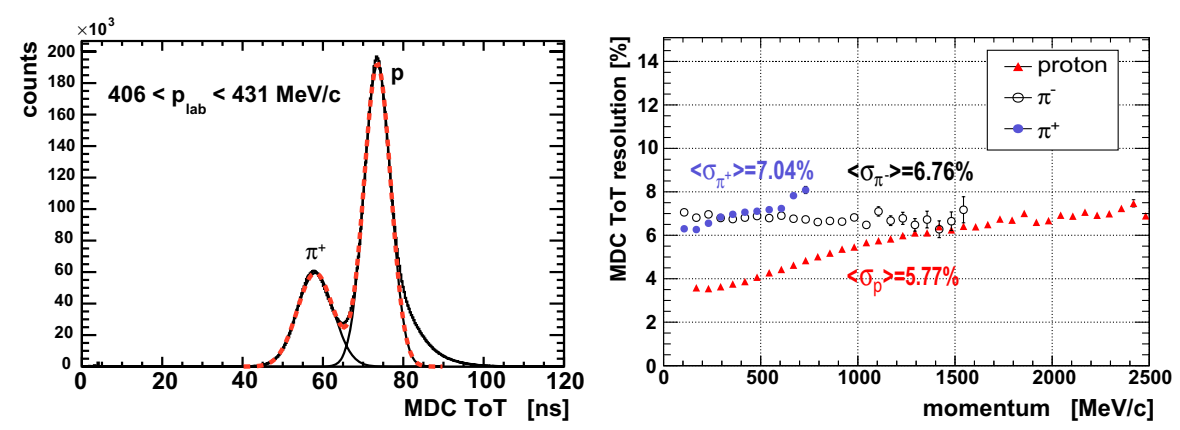

Figure 16: Left: Measured ToT distribution from the MDCs for a given momentum slice, averaged over the whole HADES acceptance. Right: ToT resolution versus particle momentum. The merging of the $\pi^{+}$line into the proton line at high momentum is due to an increasing number of wrongly identified protons.

The ToT resolution has been studied by making Gaussian fits in each $25 \mathrm{MeV} / \mathrm{c}$ wide momemtum bin (fig. [16 left). The ToT resolution for different particle species is depicted in fig. 16 right. For minimum ionizing particles, an energy loss resolution of around $7 \%$ has been achieved. A better resolution of about $4 \%$ can be found for stronger ionizing particles. The resolution strongly depends on the number of drift cell measurements contributing to a particle track. The method described here yields similar results regarding the $d E / d x$ resolution as compared to the drift chambers of Belle [29] and BABAR 30]. It fits also nicely with the empirical formula given in [31]. 


\subsection{Time-of-flight detectors}

\subsubsection{Overview}

The time-of-flight system of HADES consists of two scintillator arrays, the TOF wall for larger polar angles $\left(44^{\circ}<\theta<88^{\circ}\right)$ and the TOFINO wall for the forward region $\left(18^{\circ}<\theta<44^{\circ}\right)$. Both detectors are placed in the region behind the tracking system and they are used mainly for triggering and particle identification purposes.

A fast determination of the charged particle multiplicity of the event allows to select certain reaction classes. For heavy-ion reactions it permits impact parameter selection and hence centrality characterization. For elementary reactions it provides the possibility to enhance inelastic channels with multi-particle final states with respect to elastic scattering. Thus, the charged particle multiplicity is a natural choice for a fast first-level trigger decision. Moreover, the fast determination of the impact position of each hitting particle, spatially correlated to the position in the hadron-blind $\mathrm{RICH}$ detector, allows to perform a second-level trigger decision in order to select events which contain lepton track candidates. Both aspects will be discussed in more details in sect. 3.

The combination of the time-of-flight measurement with momentum determination provided by the tracking system and the energy-loss measurement permits to perform efficient particle identification $(\mathrm{e}, \pi, \mathrm{K}, \mathrm{p})$, which is essential for the HADES physics program.

\subsubsection{TOF}

Detector characteristics. The TOF detector 32 follows the six-fold symmetry of the whole spectrometer covering polar angles from $44^{\circ}$ up to $88^{\circ}$. Each sector consists of eight modules. Each module consists of a set of eight scintillator rods, with a total of 384 rods, which are enclosed in a carbon fiber case.

In order to reduce the probability that two particles emitted in the same collision hit the same rod (double hits) to less than $10 \%$ the TOF granularity has been matched to the charged particle multiplicity angular distribution. The rod cross section is $20 \times 20 \mathrm{~mm}^{2}$ for the innermost 192 rods and $30 \times 30 \mathrm{~mm}^{2}$ for the outermost 192. The rod length varies from $1 \mathrm{~m}$ to $2 \mathrm{~m}$, respectively, from smaller to larger polar angles.

Each rod is made of BC408 plastic scintillator material from Bicron, mainly for its optical properties: good attenuation length $(3.8 \mathrm{~m}$ in bulk) combined with high scintillation efficiency $\left(\approx 10^{4} \gamma / \mathrm{MeV}\right)$ and fast response $(2.1 \mathrm{~ns}$ decay time). Each rod end is glued to a light guide, bent by $65-67^{\circ}$ with respect to the rod itself and coupled to a photomultiplier (PMT) by means of a $2.5 \mathrm{~mm}$ thick silicone disc. The light guide has an initial square cross section in order to fit to the scintillator shape, reaching progressively a circular cross section at the PMT photocathode side. To optimize their optical properties, the rods have been wrapped in aluminized Mylar sheets, along with the light guides.

All sectors are equipped with 9133B PMTs from Electron Tubes Limited. Each PMT provides a signal from the last dynode for the amplitude measurement and from the anode for time measurement. The electronic chain includes 
a Constant Fraction Discriminator (CFD), a Logic Active Delay (LAD) and a Time to Digital Converter (TDC). The amplitude signal measurement is performed by an electronic chain consisting of a shaper and an Amplitude to Digital Converter (ADC). The signal from the CFD is split into two parts and is connected to the first-level trigger electronics chain. By performing an analog sum of all the CFD signals it is possible to trigger on the charged particle multiplicity of the collision for event selection purposes.

The time-of-flight (tof) of the particle, its hit position $(x)$ along the rod and the deposited energy $(\Delta E)$ are measured as explained below. Let us denote with $t_{\text {left }}, t_{\text {right }}$ the calibrated time intervals between the instant when the reaction occurred (given by the START detector) and the arrival of the two light pulses at each rod end, and $a_{\text {left }}, a_{\text {right }}$ are the corresponding light signal amplitudes. Then these equations can be used to calculate the following variables:

$$
\begin{gathered}
\text { tof }=\frac{1}{2}\left(t_{\text {right }}+t_{\text {left }}-\frac{L}{V_{g}}\right), \\
x=\frac{1}{2}\left(t_{\text {right }}-t_{\text {left }}\right) V_{g}, \\
\Delta E=k \sqrt{a_{\text {right }} a_{\text {left }} e^{L / \lambda_{a t}}},
\end{gathered}
$$

where $V_{g}$ is the group velocity of the light inside the scintillator rod, $\lambda_{a t}$ its attenuation length, $L$ the rod length, $k$ a constant and $x$ the hit position along the rod.
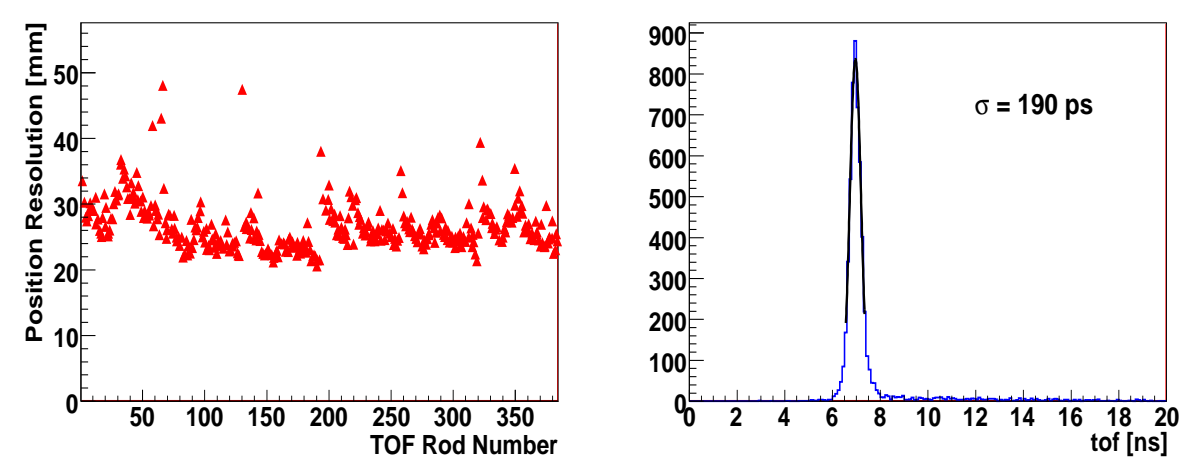

Figure 17: Left: Position resolution $\sigma_{x}$ along the rod as a function of the TOF rod number. Each group of 64 rods corresponds to one sector. Right: Time-of-flight for lepton tracks in a $\mathrm{C}+\mathrm{C}$ experiment.

Performance. The TOF wall has been running stably throughout several runs, and its performance was evaluated in $\mathrm{C}+\mathrm{C}$ and $\mathrm{p}+\mathrm{p}$ experiments. The latter 
case needs a separate treatment since it was performed without start detector (sect. 2.4.4).

The average position resolution can be determined in correlation with the MDC tracking system by taking data without magnetic field. Let us assume that a particle emitted from the target crosses MDC and TOF wall, creating useful signals in both detectors. By projecting the segment reconstructed by the MDC on the TOF system, one can calculate the position of the projected point, and its distance from the hit as measured by the scintillator rod. The distribution of the difference between the two positions $x_{T O F}-x_{M D C}$ can be fitted rod by rod by a Gaussian function, yielding the rod position offsets as well as the position resolution which is shown in fig. 17. The average resolutions along the rod $\sigma_{x}$ are $25 \mathrm{~mm}$ and $27 \mathrm{~mm}$, respectively for $20 \times 20 \mathrm{~mm}^{2}$ and $30 \times 30$ $\mathrm{mm}^{2}$ rods.

Electrons and positrons can be used to evaluate the time resolution of the TOF detector. In the energy range studied by HADES, the emitted leptons travel at velocities close to the speed of light. Their time-of-flight is then essentially independent of momentum and depends only on the traveled distance between the emission point and the TOF wall which is known from tracking. Figure [17 shows the time-of-flight distribution for lepton tracks, selected by using the spatial correlation with the hadron-blind RICH detector, normalized to the same path length of $2.1 \mathrm{~m}$. The distribution can be fitted by a Gaussian function with a width of $\sigma_{t o f} \approx 190 \mathrm{ps}$. This value depends on the time resolution of the START detector. We used an additional method in order to evaluate the TOF time resolution without this contribution, by using dileptons. If we select events which contain a lepton pair, their difference in time-of-flight should be centered at zero after correction for the path length difference and does not depend on the start time. The fit to Gaussian distributions obtained from $\mathrm{C}+\mathrm{C}$ data yields a characteristic resolution $\sigma_{T O F}$ of the $\mathrm{TOF}$ array of the order of 150 ps.

The energy loss information can be used for particle identification, but it appears to provide smaller discrimination power as compared to the time-based algorithm. For further information on energy calibration and performances see [32, 33].

\subsubsection{TOFINO}

Detector characteristics. For time-of-flight measurements at polar angles smaller than $45^{\circ}$, HADES is currently equipped by a low-granularity system called TOFINO, shown in fig. 20. It is divided into six sectors each consisting of four BC408 scintillator paddles, arranged radially with respect to the beam axis. Each scintillator has a trapezoidal form of $1.3 \mathrm{~m}$ height and is $10 \mathrm{~mm}$ thick. The light is collected from the wide side of each paddle (0.32 $\mathrm{m}$ wide) on a Hamamatsu 1949 PMT via a light guide which is bent with respect to the paddle by $65^{\circ}$.

The optical coupling between the light guide and the PMT is achieved by means of a $2.5 \mathrm{~mm}$ thick silicone disk. The paddles along with light guides have been wrapped with aluminized Mylar. Both signals from each PMT are 
used for timing and amplitude measurements using an electronic chain similar to the TOF one. The TOFINO subdetector is mounted directly in front of the Pre-Shower detector (sect. 2.5), which provides the particle hit coordinates on the paddle. In order to calculate the time-of-flight (tof) of the charged particle, the following equation is used:

$$
\text { tof }=t-\frac{x}{V_{g}}
$$

where $t$ represents the calibrated time interval between the reaction and the arrival of the light pulse at the PMT, $V_{g}$ the light group velocity in the paddle and $x$ the distance from the hit position on the paddle to the PMT.
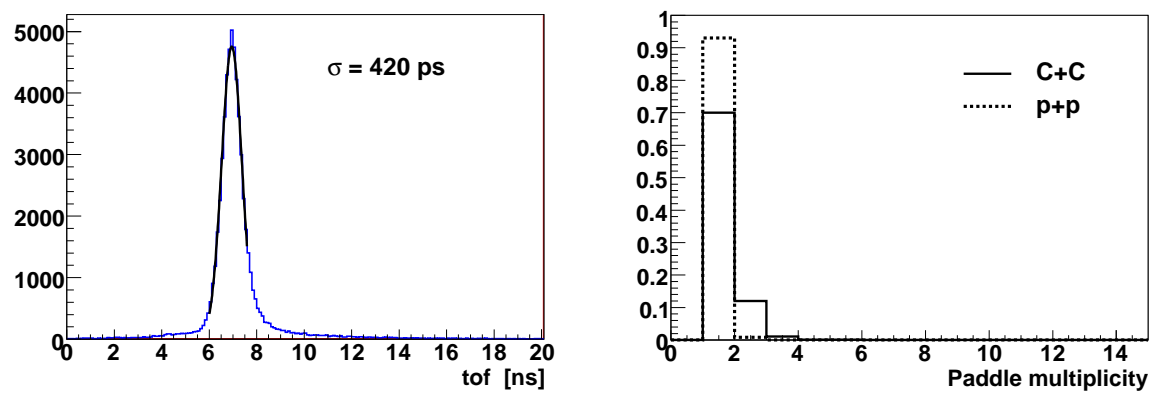

Figure 18: Left: TOFINO time-of-flight for lepton tracks from $\mathrm{C}+\mathrm{C}$ collisions. The time resolution is about 420 ps. Right: TOFINO hit multiplicities for $\mathrm{C}+\mathrm{C}$ and $\mathrm{p}+\mathrm{p}$ collisions.

Performance. The time resolution and double hit capability of TOFINO are worse than those of the TOF wall. Figure 18 shows the electron time-of-flight distribution for $\mathrm{C}+\mathrm{C}$ collisions. The time resolution of TOFINO is about 420 ps, which is determined mainly by the geometry of light collection system.

The reduced granularity increases the probability of multiple hits on the same TOFINO paddle, which hampers time-of-flight extraction for such events. The hit multiplicity depends on the reaction type as shown in fig. 18. For $\mathrm{C}+\mathrm{C}$ reactions about $15 \%$ of all events have 2 hits in one paddle, while for $\mathrm{p}+\mathrm{p}$ reactions this number drops below $0.4 \%$. The replacement of the TOFINO detector by a Resistive Plate Chamber (RPC) wall [34] is foreseen in the near future to facilitate measurements with heavy systems (e.g. $\mathrm{Au}+\mathrm{Au})$.

\subsubsection{Measurements without start detector}

For high-intensity proton beams $\left(\geq 10^{7} s^{-1}\right)$ it is not possible to use a start detector since the induced background hampers the stable RICH operation. As a consequence, there is no common start time reference for tracks in the same event. However, in this case one can measure the difference in time-of-flight with respect to the fastest particle, instead of the real time-of-flight. 
A new algorithm for time calibration of TOF and TOFINO systems as well as a procedure reconstructing the start time of the reaction have been developed [35]. For the time calibration, lepton pairs emitted in the same event were used. Assuming that both leptons travelled over the same path length, their time-offlight difference $\Delta_{\text {tof }}$ must be equal to zero and does not depend on the start signal timing. Thus by setting the $\Delta_{t o f}$ distributions to zero on a paddle-bypaddle basis, all the time offsets can be determined.

The start time reconstruction algorithm relies on the assumption that the particle identity is known from other detectors. From the assumed mass and the measured momentum, one obtains a time-of-flight estimate, and thus the offset to the real start time of the reaction. In HADES, there are two cases where such identification is unique. In the first case we use the RICH detector which is offering powerful electron/positron identification. For events without electrons or positrons we search for a negatively charged particle, which can be deduced from the track bending direction, and assume that it is a pion (obviously the most copiously produced negatively charged particle). In this way we obtain an average time resolution $\sigma_{\text {tof }}$ of $340 \mathrm{ps}$ and an efficiency of about $92 \%$ for events with a lepton, whereas a resolution of 440 ps and an efficiency of about $93 \%$ is obtained for events with a negative pion.

After the start time reconstruction it is possible to use the recalculated timeof-flight in order to identify particles for exclusive analysis of decay channels, which includes either electrons or negative pions. Figure 19] shows momentum times polarity versus velocity plots after start time reconstruction for the two different cases. As one can see, protons and pions are well resolved.
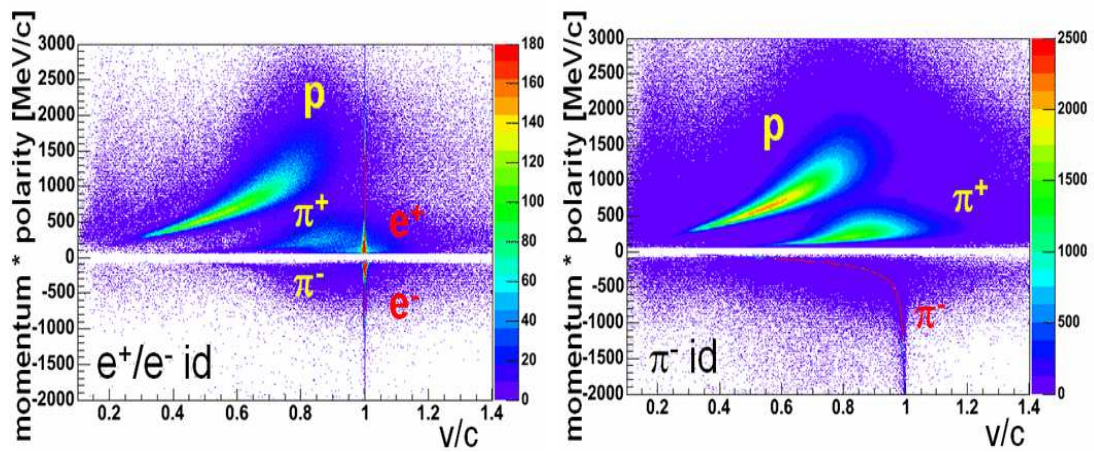

Figure 19: Left: $Z p$ vs. velocity for events from pp collisions at $2.2 \mathrm{GeV}$ with an identified $e^{+} / e^{-}$. Right: Same but with an identified $\pi^{-}$. 


\subsection{Pre-Shower}

The Pre-Shower is located just behind the TOFINO at forward angles for electromagnetic shower detection. Each sector module comprises three trapezoidal wire chambers (pre-chamber, post1-chamber, post2-chamber), separated by $\mathrm{Pb}$ converter plates as shown in fig. 20, The basic idea of electron or positron identification is schematically presented in fig. 20 (right): a charged particle passing through the gas chambers is registered by measuring the induced charge on the cathode pads with individual read-out. In order to obtain the complete charge of the electromagnetic shower an integration over several pads around the pad with the highest local charge value (local maximum) is performed. The integration is done in parallel on the corresponding pads of the three wire chambers. The comparison of the integrated charges from the different layers is the basis of the electromagnetic shower recognition algorithm, described in more detail below.

The wire chambers are filled with an isobutane-based gas mixture and are operated in the limited self-quenching streamer (SQS) mode [36, 37]. The SQS mode guarantees that an avalanche charge depends weakly on the particle specific energy loss because of the charge saturation effect limiting the chamber gain. In this mode the integrated charge is rather proportional to the number of particles traversing the chamber than to their specific energy loss. This mode of operation was selected to reduce fake contributions from non-minimum ionizing protons. Such protons produce larger energy losses in the post-converter chambers and would mimic electromagnetic showers.

\subsubsection{Detector construction}

A single sector of the Pre-Shower detector is composed of three wire chambers and two Lead converters (fig. 20) of $d_{1}=2 X_{0}$ and $d_{2}=1.5 \mathrm{X}_{0}$ thickness, respectively, where $X_{0}=0.56 \mathrm{~cm}$ is the Lead radiation length. The wire chambers are constructed identically, as shown in fig. 21, and consist of one wire plane of equally spaced cathode and anode wires positioned at $4 \mathrm{~mm}$ distance from two flat cathode planes. One cathode plane consists of a $1 \mathrm{~mm}$ stainless steel sheet. The second one is made of fiber glass with Copper cladding. The latter one is divided into 942 pads to allow individual read-out. The geometrical size of the three wire chambers, internal wire separations, pad sizes and the dimensions of the converters are scaled proportionally to achieve the same solid angle coverage for all wire chambers of a given sector. The construction parameters of the chambers were obtained from simulations aimed at assessing the optimal geometry for minimizing the double hit probability in a single pad (below $5 \%$ for $\mathrm{Au}+\mathrm{Au}$ collisions) and minimizing the number of pads involved in the charge integration procedure in order to simplify the on-line electron search. The resulting pad structure of the pre-chamber is presented in fig. 21 A detailed description of all dimensions and aspects of mechanical construction can be found in 38 .

The pads are arranged into rows (32 per chamber, see fig. 21) which are connected to front-end electronics boards, based on a dedicated ASIC chip which 


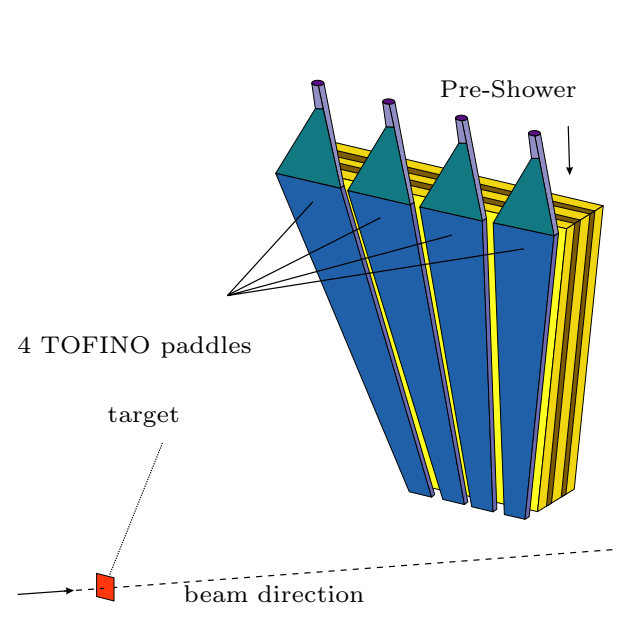

potential wires ground wires

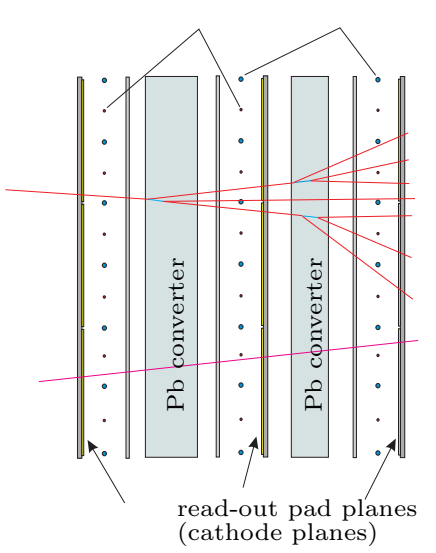

Figure 20: Left: Schematic layout of the Pre-Shower detector and forward TOFINO (one sector). Right: Side view of the Pre-Shower detector. The three gas chambers and two Lead converters are shown. Electron-hadron discrimination is performed by comparing the number of particles measured in the chambers in front of and behind the Lead converters.

represents a 32-channel charge amplifier/shaper with output multiplexer, and digitization with an 8-bit ADC [39]. In order to simplify the shower pattern recognition algorithm (sect. 3) full digital information from $32 \times 32$ pads per chamber is sent to a dedicated Image Processor Unit (IPU).

\subsubsection{Performance}

The main aim of the Pre-Shower detector is to identify electrons (positrons) by means of the electromagnetic shower detection. The electron candidate search consists of the following steps: (i) finding a local maximum of the charge distribution in the pre-chamber (hit reference position) or if not present, a local maximum of the charge in the post1-chamber, $(i i)$ integrating the charge over $3 \times 3$ pads (a local maximum pad and eight neighboring pads) in the prechamber $\left(\Sigma_{\text {pre }}\right)$, post1-chamber $\left(\Sigma_{\text {post } 1}\right)$ and post2-chamber $\left(\Sigma_{\text {post } 2}\right)$ around the local maximum and (iii) finally, applying one of the following electron identification algorithms:

a. Maximum Sum $T_{S 1}(p), T_{S 2}(p)$ :

$$
\frac{\Sigma_{\text {post } 1}}{\Sigma_{\text {pre }}} \geq T_{S 1}(p) \quad \text { or } \quad \frac{\Sigma_{\text {post } 2}}{\Sigma_{\text {pre }}} \geq T_{S 2}(p),
$$

where $T_{S 1}(p)$ and $T_{S 2}(p)$ are momentum dependent threshold values. 

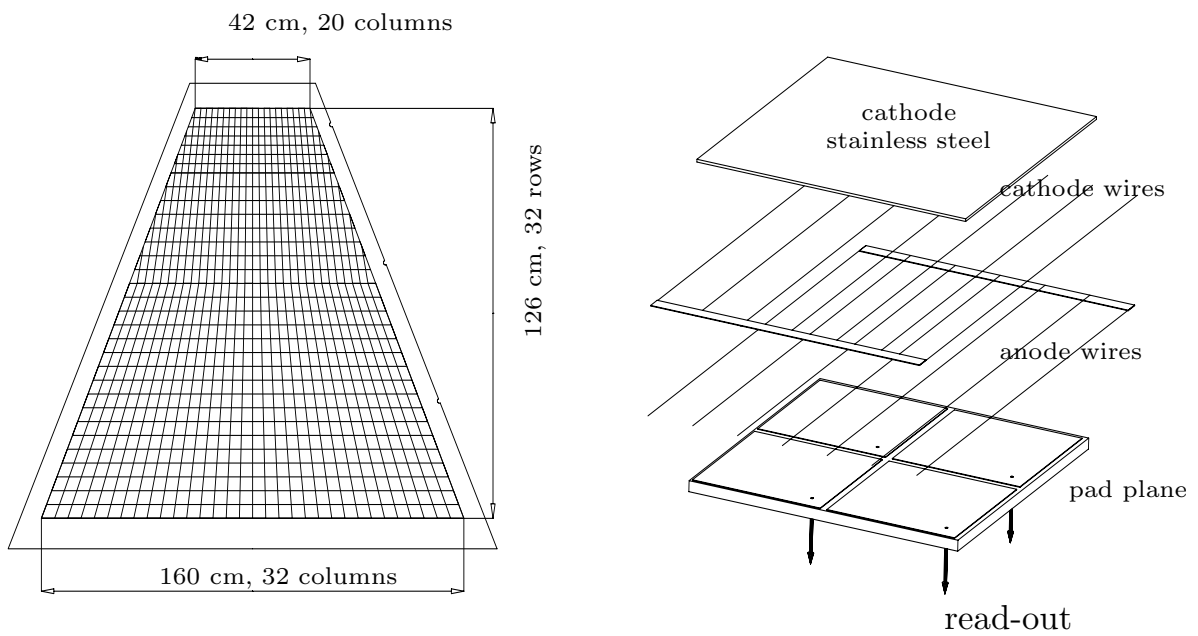

Figure 21: Left: Schematic drawing of a chamber of the Pre-Shower detector. The positionsensitive pad plane with 942 pads is shown. Right: The anode wires operating at $+2.7 \mathrm{kV}$ and cathode wires at ground potential are mounted on separate frames symmetrically positioned between two cathode planes.

b. Sum Difference:

$$
\Sigma_{p o s t 1}+\Sigma_{\text {post } 2}-\Sigma_{\text {pre }}(p) \geq T_{S D}(p) .
$$

The thresholds for both algorithms have been optimized using a dedicated Monte Carlo GEANT [4]] simulation to get the best ratio of recognized electrons to fake events (protons, pions) and to maintain high electron efficiency $(\geq 80 \%)$ over a broad momentum range $(0.1 \mathrm{GeV} / \mathrm{c} \leq \mathrm{p} \leq 1.5 \mathrm{GeV} / c)$.

Figure 23 (left) shows the Sum Difference (eq. 8 ) measured for electrons with momenta $p \approx 0.5 \mathrm{GeV} / c$ (triangles) and minimum-ionizing pions. Using these distributions the electron-hadron rejection can be evaluated for a given charge threshold as a function of momentum. The right panel of fig. 23] shows the pion rejection obtained for a threshold yielding a constant electron efficiency of $80 \%$.

The fraction of fake events is about $10 \%$ for minimum ionizing protons or pions and increases for low momentum protons. However, this can be reduced without affecting the electron identification efficiency by applying a time-offlight window of $8.7 \mathrm{~ns}$ on TOFINO hits. Using the Pre-Shower condition (eq. 8) and the time-of-flight window, more than $90 \%$ of the hadrons are rejected. Finally spatial correlations of electron candidate hits in the Pre-Shower with reconstructed tracks in the MDCs and RICH rings provide a clean electron identification (see sec. 4.4) 


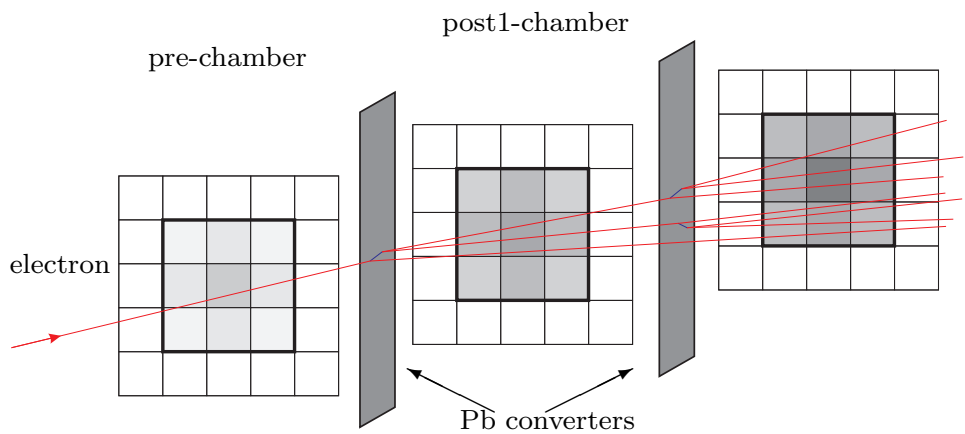

Figure 22: Schematic view of the shower algorithm. In each layer the sum of the charge over $3 \times 3$ pads is calculated. The larger charge deposition in the post-chambers is the signature of an electromagnetic shower.
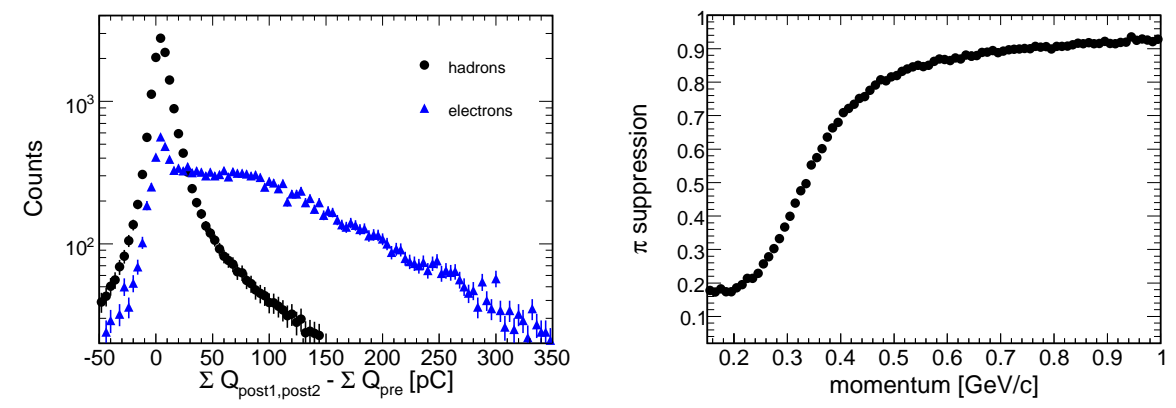

Figure 23: Left: Sum of charge $\Sigma Q$, over $3 \times 3$ pads, measured on post1- and post2-chamber with subtracted charge measured in the pre-chamber. Electrons with momenta $p \sim 0.5 \mathrm{GeV} / c$ (triangles) produce electromagnetic cascades in the $\mathrm{Pb}$ converters which results in a larger charge deposit compared to the one from hadrons, here $\pi^{-}$(dots). Right: Pion suppression as a function of momentum: the fraction of pions rejected after the algorithm for lepton recognition (electromagnetic cascade) has been applied. Data are taken from $3.5 \mathrm{GeV} \mathrm{p}+\mathrm{p}$ collisions. 


\subsection{Target and beam detectors}

\subsubsection{Overview}

The HADES physics program requires experiments with various beam-target combinations. The investigation of elementary processes leading to dielectron production is carried out with pion, proton and deuteron beams incident on a liquid hydrogen target. While the study of in-medium modifications of hadron properties at normal nuclear density requires light projectiles on heavy targets, the effect of higher temperature and compression can be investigated only with heavy projectiles. For the elementary process studies, beam detectors based on scintillating fibers have been developed. For the beam monitoring, beamprofile, time structure analysis and finally for the time-of-flight measurement for heavy-ion experiments, polycrystalline diamond detectors are employed.

\subsubsection{The liquid hydrogen target}

The liquid hydrogen target $\left(\mathrm{LH}_{2}\right)$ has been developed at IPN Orsay to fulfill the requirements for the study of elementary processes through collisions of light projectiles (pion, proton or deuteron) with protons. The target consists of a cell (50 $\mathrm{mm}$ long, $25 \mathrm{~mm}$ in diameter) filled with liquid hydrogen at atmospheric pressure and a temperature of $20 \mathrm{~K}$. The liquid is contained in a vessel built out of Mylar foils of different shapes glued together with ECO-BOND 286 glue (see fig. 24).

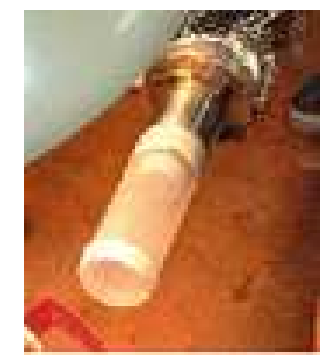

Figure 24: $\mathrm{LH}_{2}$ target. The entrance window is glued on a stainless steel tube with a diameter of $15 \mathrm{~mm}$ (visible through the transparent wall of the cell), whereas the target vessel diameter is $25 \mathrm{~mm}$.

The target entrance and exit windows as well as the cylindrical part are $100 \mu \mathrm{m}$ thick. An external carbon fiber housing, $4 \mathrm{~cm}$ in diameter and $0.5 \mathrm{~mm}$ thick, is placed around the vessel which is thermally insulated by 10 layers of superinsulation material (6 $\mu \mathrm{m}$ thick aluminized Mylar). The forward end cap of this cylindrical carbon fiber cylinder is also made out of a $100 \mu \mathrm{m}$ thick Mylar foil.

For proton-proton experiments, the interaction probability of the in-beam housing material along the beam axis amounts in total to less than $0.05 \%$, whereas the $5 \mathrm{~cm}$ long liquid part of the target yields a $0.7 \%$ interaction probability. The low energy threshold for protons to escape the target is $15 \mathrm{MeV}$ at $90^{\circ}$ and reaches $30 \mathrm{MeV}$ at forward angles. The cooling down from room 


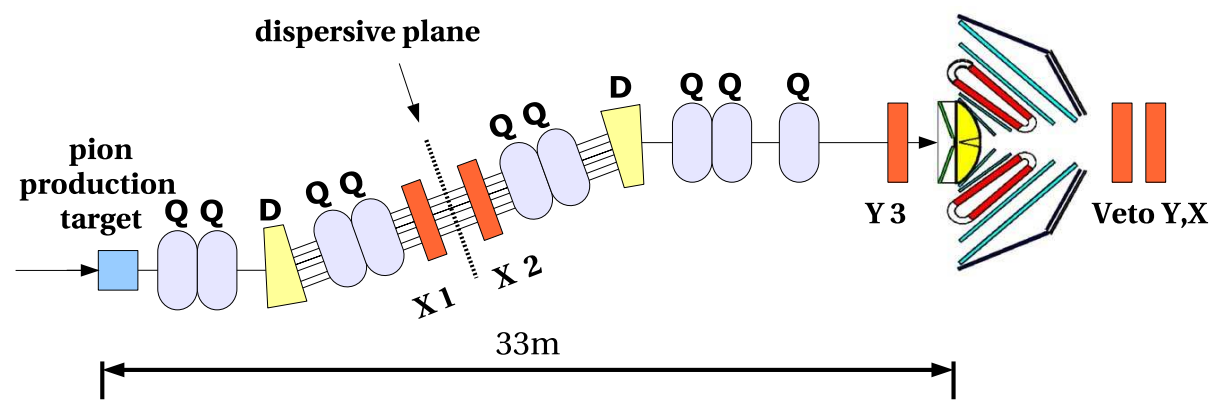

Figure 25: The beam line to HADES in a configuration for pion beams. Indicated are the positions of the pion tracking hodoscopes $\mathrm{X} 1$ and $\mathrm{X} 2$ in the dispersive plane and $\mathrm{Y} 3$ in front of the target and of the veto $\mathrm{Y}, \mathrm{X}$ behind the HADES setup.

temperature takes 12 hours. The cryogenerator operation is controlled by a Labview based interface.

\subsubsection{Light projectile beam detectors}

A production target for pion beams has been installed at the heavy-ion synchrotron SIS [41, 42]. This opens up the possibility for a broader research program at GSI, in particular at HADES, by including the study of elementary reactions which is a prerequisite for understanding the complex dynamics of heavy-ion collisions.

For minimum-ionizing pion and proton beams, the diamond START detector cannot be used since the deposited energy is too small. Therefore, alternative detector concepts are required. In addition, for secondary pion beams, a momentum measurement is mandatory for each individual pion. This is achieved by measuring the position in the dispersive plane of the beam line, shown in fig. 25. A system of five detectors is available serving as START (Y3), VETO $(\mathrm{Y}, \mathrm{X})$ and tracking devices (X1 and $\mathrm{X} 2)$ for pion beams. All detectors consist of scintillating plastic bars, strips or fibers, which are read out individually with photomultipliers. Their properties are summarized in table 3 .

The two scintillating bar hodoscopes used behind the pion production target are described in detail in [42]. Figure 26] shows a schematic cut of the Y3 fiber hodoscope consisting of 96 scintillating fibers of $1 \mathrm{~mm}$ diameter. Due to the finer granularity, fiber detectors allow to run at a higher rate of $\approx 1 \mathrm{MHz} / \mathrm{mm}$. Neighboring fibers overlap by $30 \%$ to avoid geometrical efficiency losses (closepacking, see fig. 26). To increase the light output, in particular for minimal ionizing particles, four layers of fibers are stacked behind each other. The fibers are read out via light guides with 16 -fold multi-anode photomultipliers (Hamamatsu H6568).

In the intermediate focal plane the position of pions is determined with two hodoscopes $(\mathrm{X} 1 / \mathrm{X} 2)$ of 128 scintillator strips $\left(0.8 \times 5.0 \mathrm{~mm}^{2}\right)$ each. They are read out with 32 -fold linear phototubes (HAMAMATsu H7260), whereby the signals from two adjacent strips are fed into one electronic channel. 


\begin{tabular}{|l|l|l|c|c|c|l|}
\hline label & shape & material & channels & $\begin{array}{l}\text { granularity } \\
(\mathrm{mm})\end{array}$ & $\begin{array}{l}\text { thickness } \\
(\mathrm{mm})\end{array}$ & $\begin{array}{l}\text { active area } \\
\left(\mathrm{mm}^{2}\right)\end{array}$ \\
\hline \hline X1, X2 & strips & EJ-212 & 64 & 2 & 5 & $120 \times 120$ \\
\hline Y3 & fibers & BCF99-77 & 96 & 0.7 & 5 & $67 \times 60$ \\
\hline Y, X & bars & BC404 & 16 & 10 & 5 & $160 \times 100$ \\
\hline
\end{tabular}

Table 3: Properties of the pion tracking hodoscopes. The labels refer to fig. 25
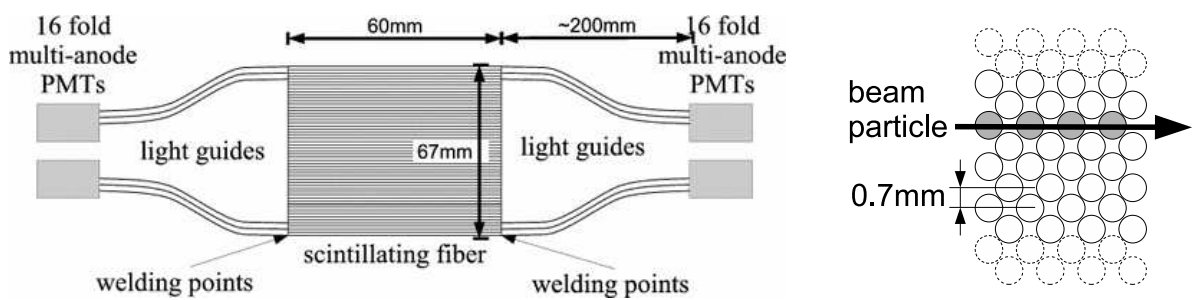

Figure 26: Left: Schematic view of the fiberplane Y3. Right: Section perpendicular to fibers.

Dedicated front-end electronics is used for the readout of the hodoscopes. Fast signals are provided for timing and triggering purposes. An estimate of the signal amplitude can be deduced from a time-over-threshold measurement.

Using $\pi^{-}$beams with a momentum of $1.17 \mathrm{GeV} / \mathrm{c}$ the efficiency of the hodoscopes has been determined to be greater than $99 \%$. The time resolution is $\sigma<300 \mathrm{ps}$. The position measurement at the dispersive plane allows a determination of the pion momentum to $\sigma_{p} / p \approx 0.1 \%$.

\subsubsection{Heavy-ion beam detectors}

Heavy-ion beam intensities up to $10^{8}$ particles/spill for ions up to $\mathrm{Au}$ and thin segmented targets (total thickness $\leq 5 \%$ of the interaction length) are being used. For this reason a START-VETO detector system made of two radiation hard CVD (Chemical Vapour Deposition) diamond strip counters has been designed. This solution has the following advantages: (i) high rate capability (signal base-width of about $2 \mathrm{~ns}$ ), (ii) radiation hardness (better than Silicon), (iii) fast signal collection time (hole mobility $1200 \mathrm{~cm}^{2} \mathrm{~V}^{-1} \mathrm{~s}^{-1}$ ), (iv) low noise (band gap $5.5 \mathrm{eV}$ ). Furthermore, no additional cooling is needed due to a thermal conductivity of $1000-2000 \mathrm{Wm}^{-1} \mathrm{~K}^{-1}$; hence the detector can be operated at room temperature.

The CVD diamond with excellent timing properties (rise time below $500 \mathrm{ps}$ ) [43] delivers a precise reference time signal for time-of-flight measurement in HADES. The HADES START-VETO system consists of two CVD diamond strip detectors located $75 \mathrm{~cm}$ upstream (START detector) and $75 \mathrm{~cm}$ downstream (VETO detector) of the target (see fig. 27). Both detectors have identi- 


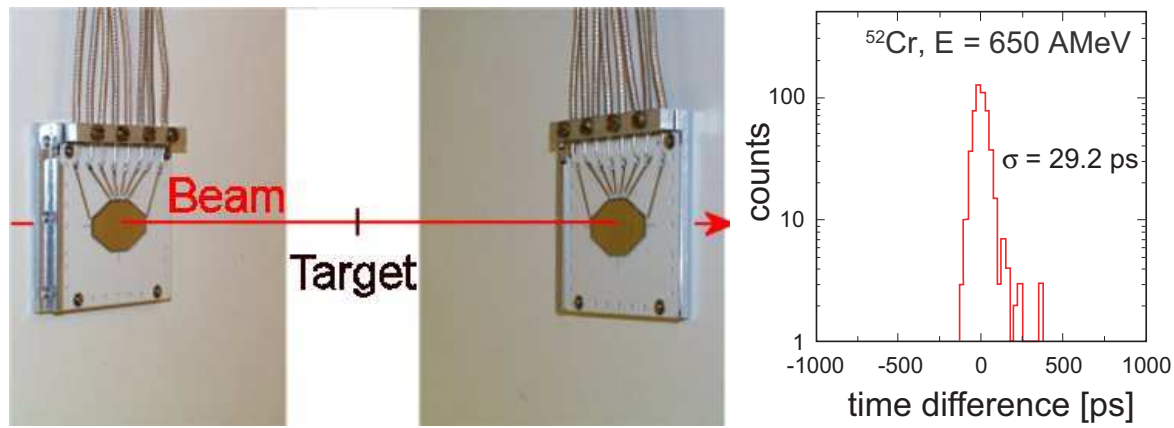

Figure 27: Left: Start-Veto system used in HADES heavy-ion experiments. Each detector consists of 8 strips with widths varying from $5.4 \mathrm{~mm}$ to $1.55 \mathrm{~mm}$. Right: Time resolution measured with $\mathrm{Cr}$ beam.

cal design, each consisting of 8 strips with variable width ranging from $5.4 \mathrm{~mm}$ for the most outer strips to $1.55 \mathrm{~mm}$ for the inner strips. The widths are optimized such that a coincidence of one Start strip with 3 Veto strips is sufficient for a Veto efficiency of $96 \%$. Furthermore, with this design the count rate per strip is nearly constant. In order to minimize secondary reactions in the start detector, the thickness of the detector is $100 \mu \mathrm{m}$ only. The time resolution of the Start-Veto system is shown in fig. 27, it amounts to $\sigma=29.2 \mathrm{ps.} \mathrm{The} \mathrm{time}$ resolution of the START-VETO system shown in fig. 27 was measured with ${ }^{52} \mathrm{Cr}$ beam at beam energy of $650 \mathrm{AMeV}$. It amounts to $\sigma=29.2 \mathrm{ps}$ and is worse for lighter beam ions like ${ }^{12} \mathrm{C}$ since the deposited energy is proportional to the $\mathrm{Z}^{2}$. The time resolution measured with ${ }^{12} \mathrm{C}$ at $2.0 \mathrm{AGeV}$ beam is about $110 \mathrm{ps}$.

Each strip of the detector is read-out by a low-noise current-sensitive broadband amplifier. The amplified signals are further processed using leading-edge discriminators with two outputs: (i) for the TDC (Time to Digital Converter) unit and (ii) for the First-Level Trigger (LVL1) logic (sect. 3). 


\section{The trigger, data acquisition, and slow control systems}

As pointed out in sect. 1. $e^{+} e^{-}$pairs arising from the decay of vector mesons are rare probes. In order to avoid the data aquisition system and the frontend electronics to be overloaded and to acquire the statistics needed for the interpretation of the electron spectra, on-line data reduction and event filtering have been used. The core of the event filtering is a two-staged trigger system reducing the amount of purely hadronic events, thus enhancing the electron yield. This strategy is outlined in the following subsections. More detailed information on the trigger system can be obtained from [44].

\subsection{Data acquisition and trigger distribution}

The trigger and data acquisition system of HADES is a distributed system. Triggers are created and transmitted to the individual subsystems from one place, the Central Trigger Unit (CTU) reacting on external trigger input sources such as multiplicity triggers, minimum bias or calibration triggers. As a result, a digital level-1 (LVL1) trigger signal is generated by the CTU, consisting of a consecutive number (trigger tag) and a trigger code containing the information about the input source which has been activated. This information is forwarded via Detector Trigger Units (DTUs) to readout modules where it is converted into sub-detector specific signals and sequences, depending on the trigger code.

After data has been read out from the front-end electronics, a level-2 (LVL2) trigger algorithm selects events by searching for electron candidates. This is done by Image Processing Units (IPUs) working on the data of the different detector sub-systems using dedicated electron recognition algorithms. The resulting hit information is combined into a single LVL2 trigger decision in the Matching Unit (MU) and then forwarded again via the CTU to all sub-systems. As the LVL2 trigger decision arrives after a latency corresponding to several events ( 5 to 10 events are typical values) the readout boards need to store the data in buffers (LVL1 pipes) large enough to hold the data for this time. After a LVL2 trigger signal has been received the data is either copied into separate memory (LVL2 pipe) or discarded, depending on the decision.

Readout programs finally transport the data (UDP network protocol is employed for this purpose) to the Event Builder (EB), a PC which combines the data from different asynchronous data sources into complete events and finally writes them to mass storage. As most of the systems are based on VME crates, these readout programs are running on standardized VME-CPUs with Linux/LynxOS. Figure 28 shows a complete overview of this concept.

\subsection{The LVL1 trigger}

The LVL1 trigger is created after one of the signal inputs of the CTU has been activated. At the same time a common start signal is provided for time measurements and serving as a source for gate signals for charge measurements. This signal is distributed to all sub-detectors within typically 500-600 ns. Meanwhile, the CTU inputs are locked until all DTUs have released the trigger bus. As a consequence, the HADES trigger system is not dead time free and the rate 


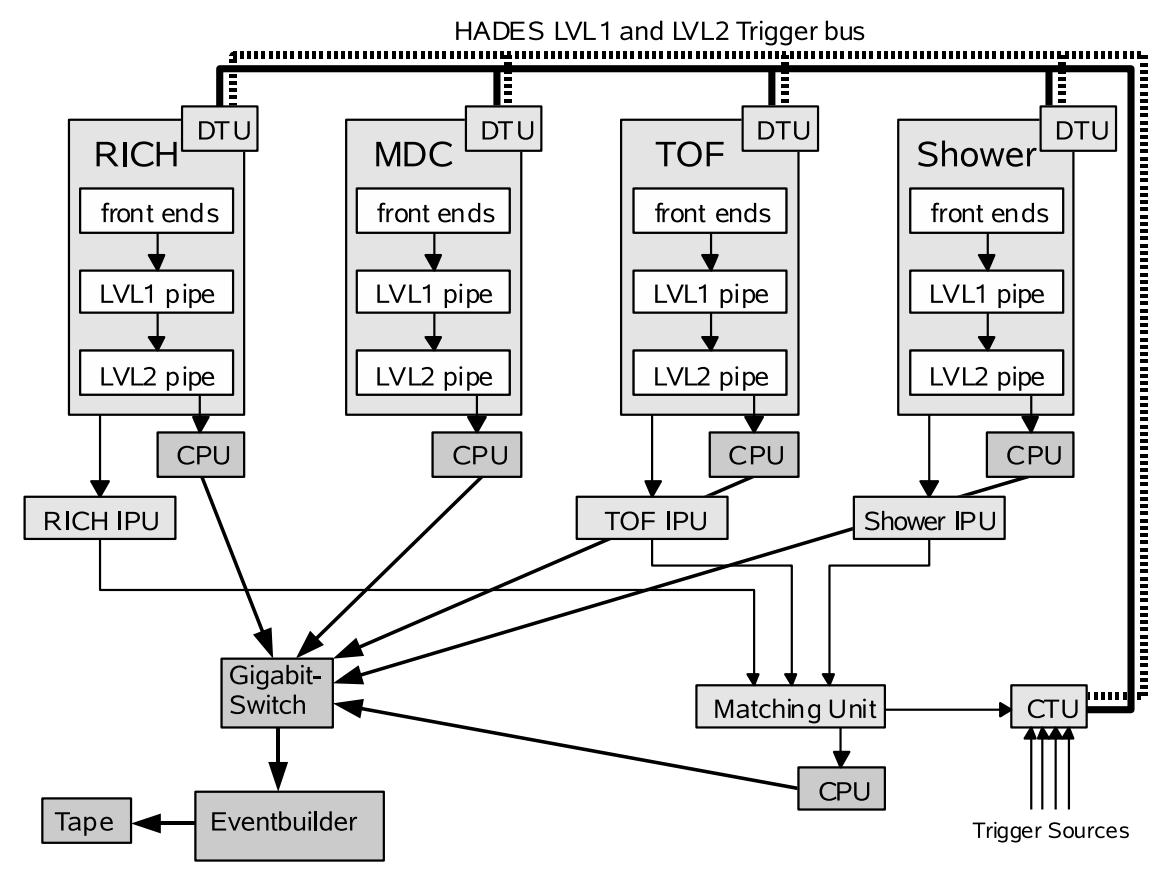

Figure 28: Block diagram of the trigger distribution and data acquisition system. Triggers are sampled by the central trigger unit (CTU) and transported via the trigger bus to the Detector Trigger Units (DTUs). The data is stored in the LVL1 pipe. At the same time pattern recognition algorithms are performed in Image Processing Units (IPUs), a Matching Unit (MU) finally combines this information. After a positive decision, the data is read out via the VME CPUs and sent to the Event Builder.

of accepted triggers strongly depends on the readout capabilities of the individual sub-systems. On the other hand, this architecture makes sure to have only complete events written to disk and no sub-detector (or part of its data) is missing.

The trigger inputs can be separated into two classes: calibration and physics triggers. In the first case, the digitization is carried out without zero suppression to monitor the time-dependent performance of the detectors, obtained with $3 \mathrm{~Hz}$ to $4 \mathrm{~Hz}$ during data taking using a pulser.

The physics trigger however is usually derived from the START detector measuring all incident beam ions in coincidence with a reaction trigger from TOF/TOFINO. In the case of a missing START detector (like in the proton runs) the first particle observed in the TOF/TOFINO detectors is used as a reference. Furthermore, as already mentioned in sect. 2.4, certain reaction classes have to be selected in order to get enhanced statistics. Besides the reaction triggers, downscaled minimum bias triggers are recorded as well e.g. to measure proton-proton elastic events for absolute cross section determination. Typical rate reductions obtained by the reaction triggers are about one order of magnitude as compared to minimum bias rates. All first-level trigger decisions are 
implemented in fast ECL logic using discrete components.

\subsection{The LVL2 trigger architecture}

The LVL2 trigger is based on two consecutive steps, which are sketched in fig. 29

In the first step each IPU is searching for either electron signatures in the data of the corresponding detector, respectively: Cherenkov rings in the RICH [14], or fast particles in the TOF, development of electromagnetic showers in the Pre-Shower or both. For each of these signatures position and angle information is provided.

In the second step the Matching Unit (MU) [45], connected to all IPUs, combines the angle information from the electron signatures of the IPUs before $(\mathrm{RICH})$ and after the magnetic field (TOF/Pre-Shower). Electron candidates are reconstructed by performing approximate tracking. Here, we exploit the fact that in first order particles are only deflected in polar direction. A narrow window in the azimuthal angle allows to correlate RICH and Pre-Shower/TOF hits. Second order corrections are taken into account by an angle-dependent matching window. The same procedure applies for positrons.

Finally, electrons and positrons are combined into pairs (dielectrons), which is done for like-sign as well as for unlike-sign pairs. The trigger conditions can be chosen in each experimental run individually. Typical requirements are at least one ring, one electron candidate or one pair per event. All units are custom-built electronics, based on programmable logic (FPGAs, CPLDs) and digital signal processors as discussed in the following sections.

\subsection{The RICH IPU}

The RICH IPU searches for possible ring centers on every pad of the squared $96 \times 96$ detector plane of a single sector, containing pads of varying dimensions (see sec. (2.2). Thanks to the special design of the photon detector pad plane the rings exhibit a constant diameter of 8 pads. The ring search is challenging due to the low photon statistics as well as by background from various sources such as charged particle hits in the photon detector and electronic noise. Therefore, several algorithms have been tested [46], where the following was chosen.

For every possible ring center on one of the $(96 \times 96=9216)$ loci, a $13 \times 13$ pad region is analyzed. All hits on a ring within a radius of 4 pads are added. There are two veto sub-regions, inside and outside the ring region, where the pads are added. These hits account to the veto region. If a certain threshold value in the ring region is exceeded and the value of the veto region is below its preset threshold, the center is assumed to be a ring candidate. The found ring centres on the $96 \times 96$ plane are then cleaned using a local maximum search. All six HADES sectors are searched in parallel. FPGA based pattern reconstruction modules and a ring recognition module have been developed. They are implemented as VME modules and obtain the hit pattern information from the $\mathrm{RICH}$ readout electronics. 


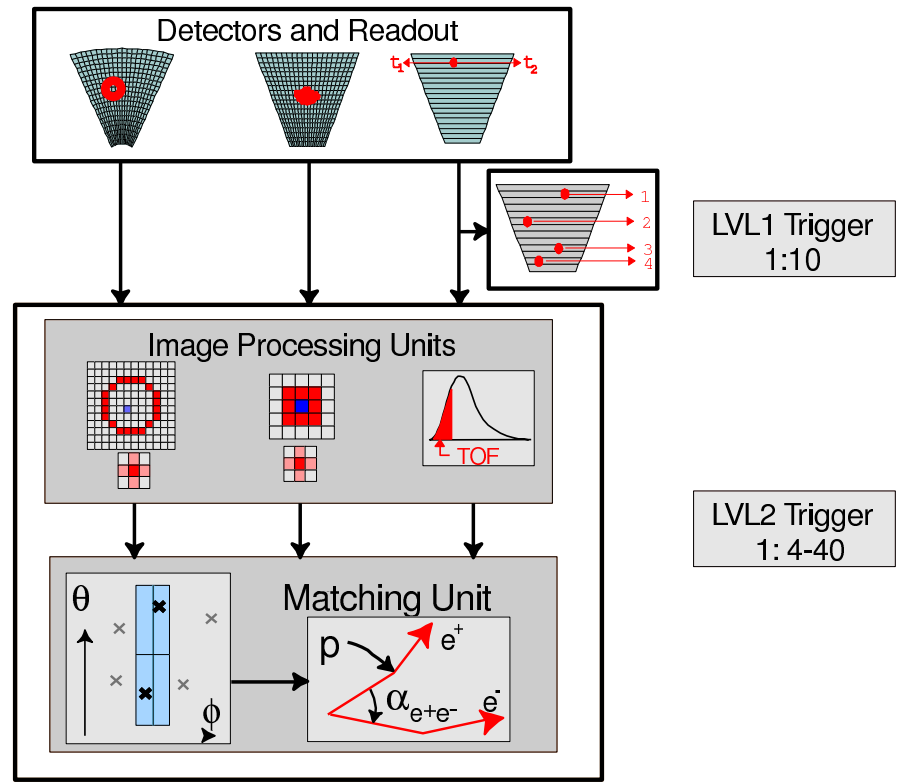

Figure 29: Overview of the reduction capabilities of the complete HADES trigger system. The first-level trigger (LVL1) is generated via charge multiplicity measurement with a certain admixture of minimum bias and calibration triggers. The second-level trigger is searching for electron hit candidates in the RICH, PreShower and TOF detectors which are correlated using the Matching Unit. The reduction factor of the LVL2 algorithm is 4 - 40, depending on the reaction under consideration.

\subsection{The Pre-Shower IPU}

The purpose of the Pre-Shower IPU is to find electron signatures in three layers of the Shower detector, i.e. in all HADES sectors in parallel, as described in sec. 2.5. The Pre-Shower IPU searches for an increase in charge of the postchamber layers, which is done by summing up the charge over $3 \times 3$ pad regions, as given by eq. 7 . Subsequently, a local maximum search is performed. The full 8 bit pulse height information is used $(3 \times 1024$ pads $)$. Additionally, the Shower IPU performs detector readout.

The realization of the Pre-Shower IPU is similar to that of the RICH IPU. The data is processed in parallel by FPGAs (Altera EPF10K100ARC), where the algorithm is implemented in a pipelined fashion, row by row. Due to the additional tasks of readout, pedestal correction, zero suppression and storage of the data in pipes for readout after a positive LVL2 decision the Pre-Shower IPU for the complete HADES detector consists of $12 \mathrm{VME}$ boards (motherboards with add-on card).

\subsection{The TOF IPU}

The Time-of-flight IPU (TIP) is a combined readout and image processing unit. The TOF readout system is also handling the TOFINO, the START detector, pion hodoscope and several other modules, like latches and scalers. 
The complete TOF readout system is based on the VME standard. Analog data from the detectors are sampled in VME based TDC/ADC modules (CAEN v878/v879). In addition, the multi-hit TDC v1190 (from CAEN) and the latch/scaler 3820 (from SIS) have been used. Here, the latch provides information about the fired LVL1 trigger source and the scaler monitores the numbers of triggers occured during the dead time of the CTU.

Each of the VME crates houses up to 18 converter modules, the VME CPU, one TIP module and one DTU. The TOF IPUs are reading out the TDC and ADC boards, which is done in $4 \mathrm{VME}$ crates in parallel. After readout on-line data analysis and image processing is performed by the IPUs. In case of a positive LVL2 trigger, data is sent via one more TOF IPU board, acting as a concentrator, to the EB.

\subsection{The trigger and readout board}

Unlike the other systems the Trigger and Readout Board (TRB) [47]) is a stand-alone ethernet based readout board replacing the v1190 module with onboard TDC functionality, based on the HPTDC [48] with the advantage to be closer to the detectors. It has been used in several experimental runs to read out beam hodoscopes as well.

\subsection{Performance of the DAQ and the LVL2 trigger}

In addition to the events containing an electron candidate as determined by the LVL2 trigger algorithm, some LVL2 trigger signals are independently marked with a preset downscaling factor as positively triggered, irrespectively of their physics contents. These events provide an unbiased event sample for a study of the LVL2 trigger algorithm properties (see below). The downscaling factor ranges from 1:3 to 1:9 depending on the event size. It was tuned to keep the reduction of the accepted LVL1 trigger rate below $10 \%$.

The performance in the LVL1 and LVL2 trigger rates are strongly coupled. For reactions with large particle multiplicities, where the DAQ system has to transport more data, the LVL2 trigger rate is limited due to the bandwidth of the readout electronics. On the other hand, the data reduction factor, related to LVL2 trigger performance, depends strongly on the collision system (background and data load) and the additional downscaling factor for the unbiased LVL2 events. Therefore, the collision system has a direct impact on the accepted LVL1 trigger rate.

Typical reduction factors are in the order of $1 / 10$. They were achieved for small collision systems including $\mathrm{C}+\mathrm{C}$ for the LVL2 trigger condition requiring at least one electron candidate found by the MU. This LVL2 setting leads typically to $17 \mathrm{kHz}$ accepted LVL1 triggers, 2 MBytes/s transported LVL2 data (at $4 \mathrm{kHz}$ ) and trigger/DAQ deadtime of nearly $100 \%$. For the experimental runs, the beam intensity was chosen to result in a DAQ deadtime of about $60 \%$. Table 4 summarizes achieved DAQ performances for the $\mathrm{C}+\mathrm{C}$ and $\mathrm{Ar}+\mathrm{KCl}$ reactions.

For reactions with large particle multiplicities, where the DAQ system has to transport more data, the LVL2 trigger rate is limited due to the design of 


\begin{tabular}{|c|c|c|c|}
\hline $\begin{array}{c}\text { beam energy } \\
\text { / system }\end{array}$ & accepted & reduction & accepted \\
LVL1 rate & factor & LVL2 rates \\
\hline \hline $2 \mathrm{AGeV} / \mathrm{C}+\mathrm{C}$ & $17 \mathrm{kHz}$ & $12-20$ & $4 \mathrm{kHz} \simeq 2 \mathrm{MBytes} / \mathrm{s}$ \\
$1.76 \mathrm{AGeV} / \mathrm{Ar}+\mathrm{KCl}$ & $7 \mathrm{kHz}$ & $3-4$ & $1-2 \mathrm{kHz} \simeq 7-14 \mathrm{MBytes} / \mathrm{s}$ \\
\hline
\end{tabular}

Table 4: DAQ and trigger performance for typical in-beam conditions.

the data paths of the readout electronics, which is one of the reasons for the ongoing upgrade project [47].

However, one should note that the accepted trigger rates are approximately a factor of two smaller than the equivalent LVL1 rates when a random pulser is used. The reason is traced back to short-time $(10-150 \mu \mathrm{s})$ beam intensity fluctuations caused by the extraction system resulting in a reduced effective duty cycle and a larger dead-time.

For the above conditions we found electron pair efficiencies of about $90 \%$, in good agreement with the trigger emulation using the off-line analysis [49]. No physics bias is introduced by the second-level trigger, as will be discussed in sect. 4.

\subsection{Slow control}

The slow control system is based on the EPICS [50] control system. We use the EPICS toolkit to build the necessary client and server programs which monitor and control the hardware of the experiment. As much as possible, applications available in the EPICS community were reused and only the parts for our custom hardware were written from scratch. Our development included the driver for CAMAC via VSB bus, high-voltage control with a LeCroy 1440 system, driven via CAMAC, readout of a custom temperature monitoring system, and readout of gauges for gas bottles via serial lines. In addition, many types of genSUB records were produced to control VMEbus-based custom hardware. Some devices were designed using the field bus CANbus for crate control, for control of low-voltage regulation boards, for power supplies, and for the fast RICH current monitor.

On the client side, we use the common tools for user, namely a graphical user interface, an alarm handler and a backup and restore tool. To store values of read-back parameters the ChannelArchiver tool is used. The stored data can be used for reconstructing gains and detector efficiencies. A more powerful means of storage than the internal file format of the Archiver was developed at SLAC, the OracleArchiver, which has been adapted to our needs and extended to provide summing and mean value generation during runs. 


\section{Data analysis and detector performance}

\subsection{Framework}

The HADES on-line/off-line analysis is realized within the HYDRA framework [51], i.e. the Hades sYstem for Data Reduction and Analysis, based entirely on the $\mathrm{C}^{++}$class package ROOT [52]. This approach allows full and consistent use of all built-in features of the ROOT software developed and maintained at CERN, and which has meanwhile become a de facto standard in most high energy and nuclear physics experiments. The object-oriented design chosen for HYDRA is modular, which means that detector-specific and/or task-specific classes are all derived from a common set of base classes, and it is therefore very flexible and extendable. Input can be taken from different data sources, namely event servers, list-mode data files in various stages of analysis, data summary files or simulation files. The execution flow of the data processing is realized via freely configurable task lists, controlled via ROOT user macros. The initialization of geometry, set up and calibration parameters is possible from an Oracle data base [53] and/or from ROOT files, with full version management implemented. The analysis can be run in stand-alone batch mode or from interactive ROOT sessions. Indeed, the developed analysis code is routinely used, both for off-line and on-line data processing during data-taking runs. In the on-line mode, convenient control of the basic functionality is available via a Graphical User Interface implemented with the Qt widget library [54]. Details of the HYDRA class design and implementation are given in [51].

For simulation studies, the analysis is interfaced to the detector simulation package GEANT3 [40] from CERN via ROOT event files that are read and digitized by HYDRA. Event overlay, i.e. the embedding of simulated tracks into real events for efficiency and performance investigations, is supported as well. Finally, a comprehensive and modular ROOT-based event generator, called $\mathrm{PLUTO}^{++}$[55, 56], has been developed for fast simulation studies, but also as an input source for detailed GEANT simulations.

The HYDRA and PLUTO ${ }^{++}$frameworks have been implemented to run on various flavours of the Linux operating system. Apart from the (freely available) Oracle data base client, only open-source software has been used.

In the following subsections we present the aspects of the high-level data analysis which are specific to HADES experiments, namely the track reconstruction algorithms, the particle identification (hadron and lepton) procedures, and the dielectron reconstruction procedure. The description of the specific analysis packages needed for elementary reactions, such as the event hypothesis and the kinematic refit, will be part of a future publication as they go beyond the scope of the present paper.

\subsection{Track reconstruction}

The reconstruction of the particle trajectories in the tracking system of HADES is accomplished in several steps: 
1. The spatial correlation of fired drift cells in the drift chambers (MDCs) is performed by a track candidate search (sect. 4.2.1) based on the identification of so-called wire clusters. The wire clusters are defined using only the geometrical positions of the fired drift cells and define track segments. Track candidates are finally obtained through the matching of track segments in the inner and outer drift chambers within one sector.

2. The corresponding space positions of the track candidates are fitted by a model function taking into account the drift time information of the cells (see sect. 4.2.2).

3. For electron identification, the inner track segments are matched with rings in the RICH detector, while for all tracks the outer track segments are matched with hit points in the TOF or TOFINO and Pre-Shower detectors.

4. The particle momentum is determined by various algorithms making use of the bending of its trajectory inside the magnetic field region (see sect.4.3).

5. Particle identification is supplemented by the information of the particle momentum, its time of flight and energy loss in the TOF/TOFINO and the MDCs. Furthermore, the correlation with a reconstructed ring in the RICH detector and a detected electron shower in the Pre-Shower detector provide an efficient lepton identification.

\subsubsection{Track candidate search}

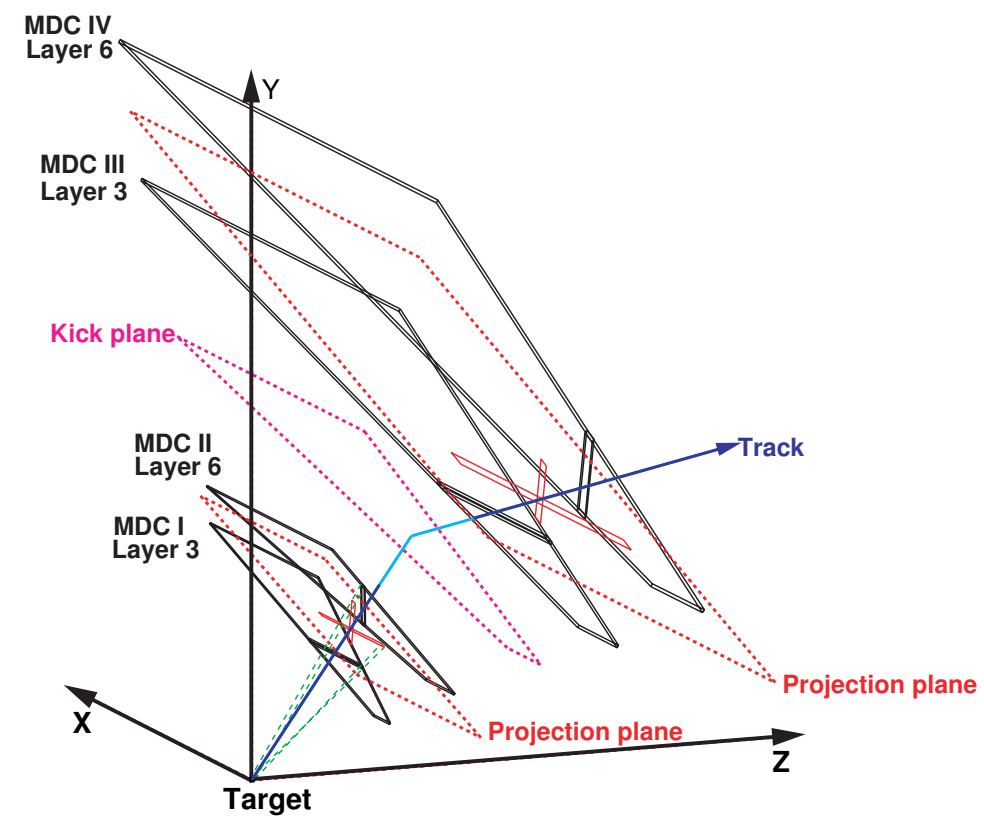

Figure 30: Principle of the track candidate search in the track reconstruction procedure. For an easy view, only one layer is shown in each MDC 
The track candidate search is based on the projection of the area of fired drift cells along a certain direction onto a common projection plane and the localization of maxima in this plane. The projection plane chosen (see fig. 301) is the centre plane of two coplanar outer chambers, while for the non-coplanar inner geometry the plane is chosen such that the projections of the drift cells are of similar size.

For the inner drift chambers the projection is performed with respect to the centre of the target. Here, only the extension of the target along the beam axis is taken into account. The impact point of the track on the projection plane is given by the local maximum of two-dimensional distributions built from the slices spanned by fired drift cells, as shown in fig. 31 [57]. To reduce the number of fake candidates created by accidentally crossing hit wires, all fired drift cells belonging to either one or both inner chambers are simultaneously projected onto one plane. The target position and the location of the maximum in the projection plane then define a straight track segment in space.

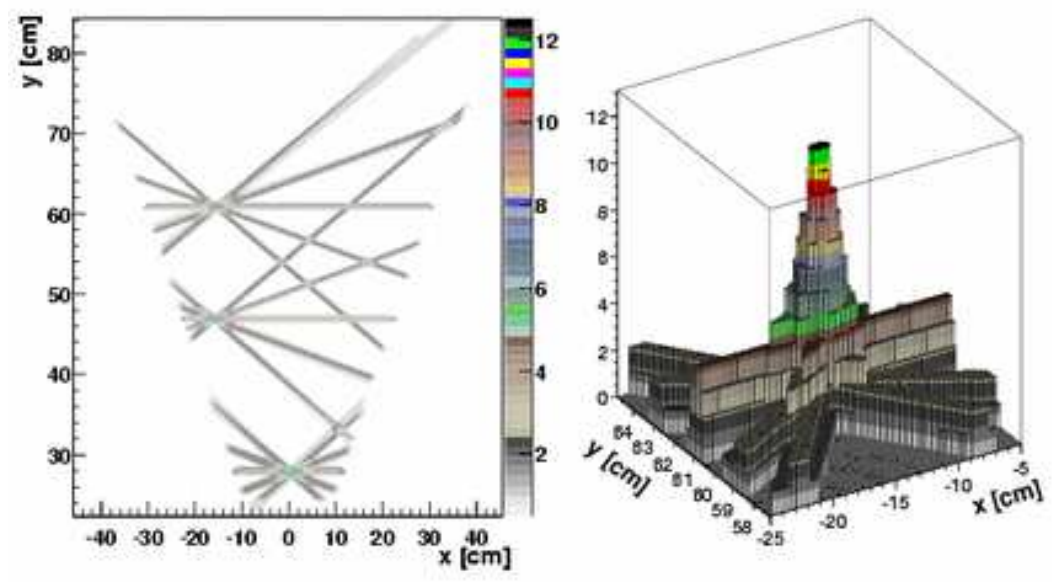

Figure 31: Left: $x-y$ detector coordinate space projection of the drift cells in the cluster finding procedure. Right: 2-dimensional histogram with a peak at the location where the drift cell projections have maximum overlap. In this example, the $z$ axis indicates the peak height, corresponding here to a track totalling 12 hit layers in the inner drift chambers [57].

The deflection of a charged particle by the toroidal magnetic field of the HADES magnet can be approximated by a momentum kick on a nearly flat virtual kick plane [51] in the field region (see sect.4.3.1). Hence, when searching for wire clusters in the outer drift chambers, the same strategy is followed as for the inner ones, except that the target position is replaced by the intersection point of an inner segment with the virtual momentum kick plane. This inherent matching of inner and outer segments defines a track candidate. This is shown in fig. 30 which depicts a schematic representation of the candidate search. Note that this procedure neglects the additional deflection due to weak fringe fields 
reaching into the MDC volumes.

The spatial resolution of the track candidate search is defined by the wire spacing. The stereo angles of the wire planes have been optimized for best resolution in the direction of particle deflection. Thus, the position resolution is worse along the $x$ coordinate of the chamber than on the $y$ coordinate. For the inner drift chambers the resolution along the $x$ coordinate is $1.12-1.5 \mathrm{~mm}$ and along the $y$ coordinate $0.8-1 \mathrm{~mm}$. Due to their larger cell size, the resolution in the outer drift chambers is $3.9-4.8 \mathrm{~mm}$ and $2.9-3.3 \mathrm{~mm}$ in $x$ and $y$ directions respectively.

\subsubsection{Track segment fitting}

The precision of the reconstructed hit points in space is improved from a few $\mathrm{mm}$ down to typically $0.1-0.2 \mathrm{~mm}$ by making use of the drift time measurement and by fitting the space coordinates of the track to a track model. This requires to convert a measured drift time into a distance to the sense wire. The distance to time correlation for each drift cell geometry ( $x-t$ correlation) was obtained from GARFIELD [58] simulations and checked against test measurements [59].

The drift time measured by the TDC connected to a drift cell is the sum of the real drift time, the propagation time of the signal along the sense wire up to the readout electronics and the time of flight of the particle to the drift cell relative to the stop signal derived from the trigger. The propagation time of the signal can be subtracted from the measured time since it can be computed from the known position of the hit on the wire. As the remaining time value still contains a part due to the time of flight of the particle, it is suitable to perform a track model fit with respect to a time variable, simultaneously extracting the time of flight of the particle.

Fitting two chambers simultaneously is performed employing a straight-linetrack model. Again, we neglect the residual fringe fields in the chamber region. However, since only the coordinates of the hit points at each chamber mid plane are used in the final momentum determination, the relative small bending effect between the two chambers is a second order effect and can be neglected. The following function $F$ for the least square minimization is evaluated for all drift cells of a track segment [60]:

$$
F=\sum_{i} \frac{\left(t_{d r i f t}^{i}+t_{s h i f t}-t_{T D C}^{i}\right)^{2}}{\left(\Delta_{T D C}^{i}\right)^{2}} w_{i},
$$

$$
\begin{aligned}
t_{\text {drift }}^{i}: & \text { drift time from the GARFIELD model, } \\
t_{\text {shift }}: & \text { time shift of all cells, } \\
t_{T D C}^{i}: & \text { measured drift time (after proper TDC calibration), } \\
w_{i}: & \text { weighting constant (Tukey weight), } \\
\Delta_{T D C}^{i}: & \text { error of drift time measurement, }
\end{aligned}
$$


where $i$ labels the individual drift cells and runs over all cells in the track segment.

In the first iteration, the time shift $t_{\text {shift }}$ is calculated as the mean deviation of all drift time measurements from the known $x-t$ correlation. This constant, determined independently for each particle track in an event, contains the time of flight of the particle to the drift chamber, as well as deviations which are common to all drift cells considered in the fit. Those can originate from errors in the determination of the calibration coefficients as well as from changes in the operating conditions of the chambers which modify the $x-t$ correlation of the drift cells. Individual deviations (time offsets, calibration parameters, high-voltage operating conditions) among the layers which are used for a given track fit are not taken into account by this constant and therefore, they contribute directly to the deterioration of the resolution. The error of the drift time measurement $\Delta_{T D C}$, as used in the functional $F$, is taken from the GARFIELD simulations.

The weighting factors $w_{i}$ are calculated according to a Tukey weight distribution [61]. The weight serves to minimize the influence of outliers (like uncorrelated noise or drift time measurements belonging to another track) on the fit result. It is evaluated dynamically at each step from the difference between the calculated drift time and the measured one.

\subsection{Momentum determination}

The HADES data analysis employs three different momentum reconstruction algorithms. The kick plane method provides a fast and robust estimate of the particle momentum with limited resolution using reconstructed inner track segments and hit points on the META detector only. With better precision, as well as moderate computational requirements, the spline method obtains the particle momentum from matched reconstructed inner and outer track segments. Finally, the Runge-Kutta method provides the best precision in reconstructing the particle momentum, but requires more computational resources. Whereas kick plane can provide a momentum at order zero in case of no outer MDCs (experiments done before 2004), Spline and Runge-Kutta are the standard methods to reconstruct the momentum. Spline provides first guess momentum and particle polarity which are then used for the iterative Runge-Kutta. All three momentum algorithms are applied from the lowest to the highest precision level, each step providing a starting value for the next one with the needed accuracy. The results of all algorithms are stored in parallel, allowing for a detailed monitoring of the procedure.

\subsubsection{Kick plane}

Schematically, the progressive deflection of a charged particle on its way through the toroidal field of the HADES magnet can be substituted by a single kick occurring on a two-dimensional, almost flat virtual surface, called kick plane [51]. This surface is determined in ray-tracing simulations using GEANT3 and corresponds roughly to the center plane of the magnetic field in any given 
sector of HADES. Within the kick plane approach, the momentum $p$ of a deflected particle is obtained in a straightforward way from its deflection angle via pre-computed look-up tables. This approach provides a very fast initial value of $p$, to be used as starting point in more refined track fitting algorithms (see below).

In general, the momentum change of a particle in the field, i.e. the momentum kick, can be written as

$$
\left|\overrightarrow{p_{\text {in }}}-\overrightarrow{p_{\text {out }}}\right|=\left|p_{k}\right|=2 p \sin \left(\Delta \theta_{k} / 2\right)
$$

where $\Delta \theta_{k}$ is the deflection angle in the bending plane, and $\overrightarrow{p_{i n}}$ and $\overrightarrow{p_{\text {out }}}$ are the incoming and outgoing three-momentum vectors, both of magnitude $p$. For large momenta (i.e. small deflections) one can set $2 \sin \left(\Delta \theta_{k} / 2\right) \simeq \Delta \theta_{k}$ and hence the momentum kick is approximated by

$$
\left|p_{k}\right| \simeq p \Delta \theta_{k} .
$$

On the other hand, the total deflection is given by the integral of the Lorentz force acting on the particle of charge $q$ on its way through the magnet

$$
\Delta \theta_{k}=\frac{q}{p} \int_{l_{\text {in }}}^{l_{\text {out }}} B \sin \alpha d l=K\left(l_{\text {out }}-l_{\text {in }}\right)=f\left(\sin \left(\Delta \theta_{k} / 2\right)\right),
$$

where $\alpha$ is the angle between the particle trajectory and the magnetic field, $K$ is a constant depending on particle polar and azimuthal angle. For the HADES field configuration, $\sin \alpha \approx 1$ and $\left|p_{k}\right| \simeq \int B(l) d l$ is a function of the path length, i.e. it depends at first order on the particle polar and azimuthal emission angles but also at second order on the momentum.

Performing a Taylor expansion in $\sin \left(\Delta \theta_{k} / 2\right)$ of eq. 12, keeping only terms up to second order, one obtains

$$
a+b \sin \left(\Delta \theta_{k} / 2\right)+c \sin ^{2}\left(\Delta \theta_{k} / 2\right)
$$

which finally, with eq. 10, gives the result 51]

$$
p=\frac{1}{2} \frac{p_{k 0}}{\sin \left(\Delta \theta_{k} / 2\right)}+p_{k 1}+2 p_{k 2} \sin \left(\Delta \theta_{k} / 2\right),
$$

where $\mathrm{p}_{k 0}$ can be interpreted as the $0^{t h}$-order momentum kick. The parameters $\mathrm{p}_{k 0}, \mathrm{p}_{k 1}, \mathrm{p}_{k 2}$ depend only on the entry and exit points of the particle track in the magnetic field; they are stored in look-up tables as function of polar and azimuthal angles.

Besides its computational simplicity and speed, the kick-plane method has also the advantage that it can even be applied when only position information from the META detector is available. In that case, the limited META position resolution $\left(\sigma_{M E T A}=6-13 \mathrm{~mm}\right)$ dominates the momentum resolution $\sigma_{p} / p$, which goes from about $2 \%$ for $0.15 \mathrm{GeV} / \mathrm{c}$ electrons, up to values of about $15-18 \%$ for $1.4 \mathrm{GeV} / \mathrm{c}$ electrons, slightly depending on the polar angle. 


\subsubsection{Spline method}

In the framework of spline momentum reconstruction method, a cubic spline is taken as a model for the particle trajectory through the magnetic field. The spline provides a smooth curve passing through the detector hit points, as well as smooth first- and second-order spatial derivatives at the given hit points. As an input, this algorithm employs track candidates containing inner and outer track segments. They are used to calculate intersection points of the reconstructed segments with the chamber mid-planes. Using four such intersection points in space, a cubic spline function in the $\left(z, r=\sqrt{x^{2}+y^{2}}\right)$ plane is applied. Fifty equally-spaced points are selected and the corresponding derivatives are calculated. The magnetic field strength is computed at the selected points using the three-dimensional HADES magnetic field map (see fig. 32).

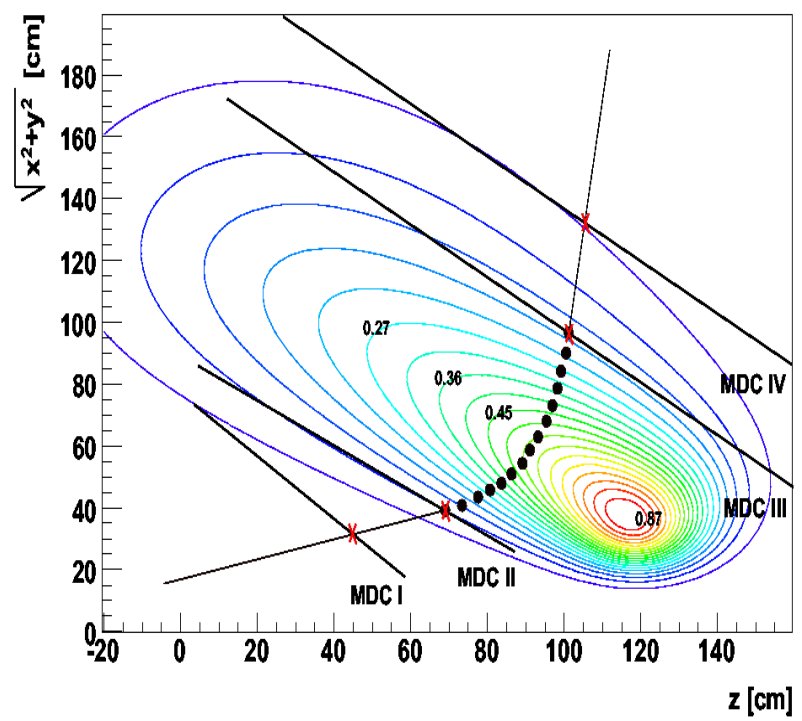

Figure 32: The track as modelized by a cubic spline in the plane defined by the threemomentum at the target and the beam axis. The 50 points (only 15 are shown here for clarity) run from MDCII up to MDCIII.

By minimizing the squared deviations $d^{2}=\left(r_{\text {spline }}-r_{\text {field }}\right)^{2}$ between the spline abscissa $r_{\text {spline }}$ and the one obtained from GEANT simulations $r_{\text {field }}$ this one being momentum dependent - one gets a momentum value at each of the 50 points. By averaging over the full set of selected points, a momentum is 
determined [61, 62]. However, since a cubic spline does not necessarily exactly model the trajectory of a charged particle through the magnetic field, systematic corrections, determined through GEANT simulations and parameterized, have to be applied in order to achieve sufficient resolution. With these corrections, the relative momentum resolution $\sigma_{p} / p$ of the method, as obtained from simulations, is ranging from $1.5 \%$ to $4.5 \%$ for $0.15 \mathrm{GeV} / \mathrm{c}$ electrons in the $\theta$ range $\left[20^{\circ}-80^{\circ}\right.$ ], decreasing to values ranging from $1 \%$ to $2.8 \%$ for $1.4 \mathrm{GeV} / \mathrm{c}$ electrons in the given angular range.

\subsubsection{Runge-Kutta method}

The standard method to determine the momentum of a particle traversing a known magnetic field consists in solving its equations of motion in the field region. In general, the system of second-order differential equations is handled by the fourth-order Runge-Kutta method of Nystrom in a recursive way [63]. The numerical solution of a differential equation requires, however, initial conditions of the function and its first derivatives, provided in our track reconstruction by the spline method (momentum and polarity) and the track-segment fitter (vertex and direction). The track parameters $-x, y$ at $z=0$. and two direction cosines in the MDCI chamber coordinate system and the momentum $p$ - are iteratively optimized to fit to the hit points measured in the MDCs.
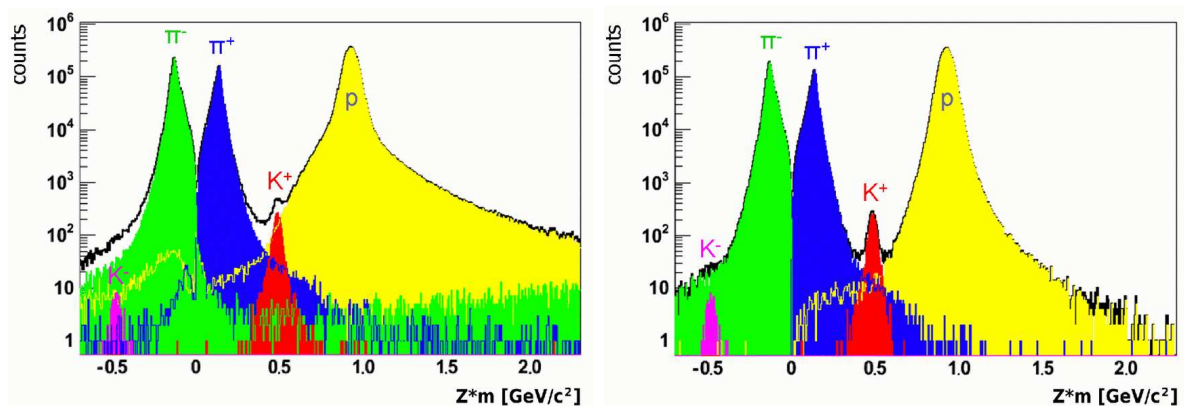

Figure 33: Left: Charge $Z$ times mass distribution using the Runge Kutta method. No selection is made on the track quality. Right: Same distribution with a track quality cut $\chi_{R K}^{2}=5$. These spectra are built out of tracks from $71 \cdot 10^{6}$ events obtained from full GEANT simulations of $2 \mathrm{AGeV} \mathrm{C}+\mathrm{C}$ collisions in the UrQMD model. Different particle species are indicated by different colors. Details are given in [64].

Presently our Runge-Kutta method does neither take into account the energy loss of the particle nor its multiple scattering. This is however acceptable as the total material budget (MDCI to MDCIV) stays typically below $0.5 \%$ radiation length. Furthermore, the procedure assumes presently that the error matrix attached to any given MDC hit is diagonal, i.e. that the parameters characterizing a given hit are all uncorrelated. A least-square minimization procedure solves the linear equations with respect to the track parameters.

As the result of Runge-Kutta tracking, parameters of charged particle trajectory are estimated, i.e. momentum and the initial direction vector. In addition 
to that, a specific $\chi_{R K}^{2}$ value is provided which can be used either as a criterion for track quality selection or as a method for particle identification [64]. This is especially important for the identification of rare tracks like $K^{+}$and $K^{-}$(see fig. 33), which shows mass times polarity distributions of simulated tracks for $\mathrm{C}+\mathrm{C}$ collisons at $2 \mathrm{AGeV}$ with (right) and without (left) track quality selection.

\subsubsection{Momentum resolution}

Data from proton-proton elastic collisions at $3.5 \mathrm{GeV}$ have been used to investigate the dependence of the momentum resolution on the particle momentum. As the kinematics is a two-body one, and since the angular resolution does not contribute significantly to the momentum resolution, the momenta of the scattered protons can be calculated almost exactly from the reconstructed polar angle. From the residual of the momentum derived from the reconstructed polar angle and the reconstructed Runge-Kutta momentum, integrated over all particle tracks, a resolution of about $4 \%$ has been obtained (fig. 35 left).

Figure 34: Simulated momentum resolution for protons with realistic errors (GARFIELD errors multiplied by 4) shown for the RungeKutta method (full symbols) and compared to the case without applying any detector resolution ('RungeKutta ideal'; open symbols) for 2 different polar angles.

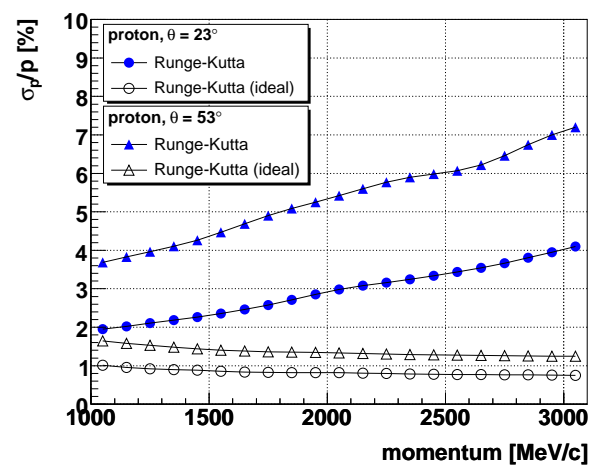

Under the same conditions a full-featured GEANT simulation, based on the time resolution from GARFIELD calculations, would give an average momentum resolution of $1.5 \%$. Figure 34 shows the simulated resolution for protons at two laboratory polar angles. The 'Runge-Kutta ideal' curve corresponds to the case where the detector resolution is not included, i.e. one sees only the effect of the multiple scattering. As one can see spatial resolution plays a dominant role for $p>1000 \mathrm{MeV} / \mathrm{c}$.

The 'Runge-Kutta' curves in fig. 35, close to experimental data, include the realistic detector resolutions, obtained by scaling-up by a factor 4 the nominal GARFIELD resolution values. At first glance, the apparent disagreement with the GARFIELD calculations is in conflict with results shown on fig. 12, However, since the wires of the two 0 degree layers are parallel, the width of the correlation patterns shown on fig. 12 is independent of any offset (wire geometrical misalignment or miscalibration) of the two adjacent wires. When reconstructing a hit on a chamber with 6 layers and over areas greater than typically the cell size squared, one averages over many wires and then gets sensitive to several individual and uncorrelated offsets. This leads to the observed deterioration of 
the resolution. Disentangling the respective role of the different offsets is still under investigation.
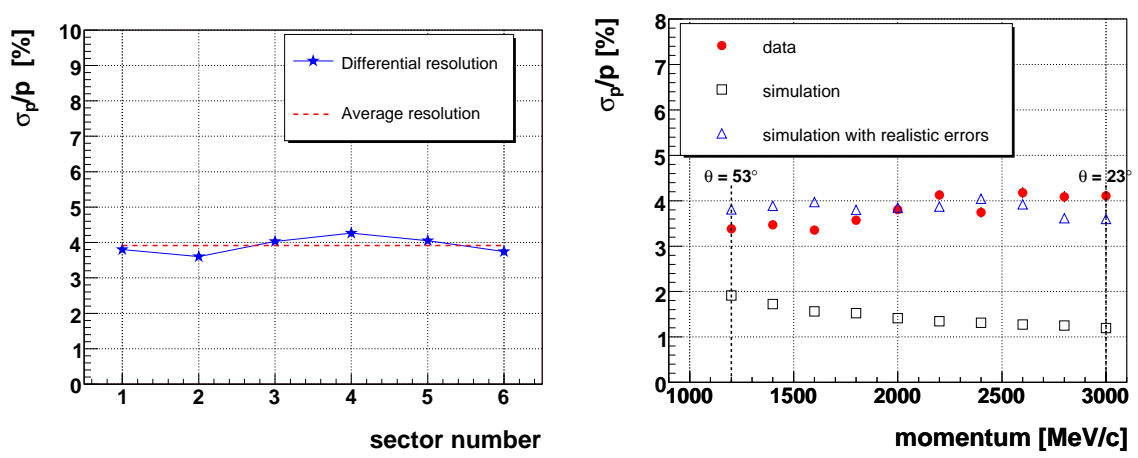

Figure 35: Left: Integrated momentum resolution for the 6 sectors of HADES. Right: Momentum resolution as a function of momentum. A full featured GEANT simulation assuming the drift cell resolution obtained from GARFIELD simulations [59] (black open squares) and a four times worse resolution to account for uncertainties originating from calibration and alignment (blue open triangles) is also shown. In both figures, $3.5 \mathrm{GeV}$ proton-proton elastic events were used. Elastic scattering laboratory angles corresponding to the momentum range shown are indicated.

After this renormalisation, a satisfactory agreement between the observed resolution and the simulation is obtained (fig. 35 right). However, the large effect seen on the protons turns out to be of much less importance for dielectron spectroscopy, which is the goal HADES was built for. The average momentum of electrons or positrons, about $0.4-0.5 \mathrm{GeV} / \mathrm{c}$ from the $\omega$ decay, is much lower than the proton average one and the effect of multiple scattering is then dominant for electrons, as can be seen on the fig. 36 left. Even with the realistic resolution, i.e. detector resolution scaled up by a factor 4 , the relative worsening of the resolution stays below $40 \%$. Figure 36 right displays the simulated resolution under realistic conditions for $0.3 \mathrm{GeV} / \mathrm{c}$ electrons versus laboratory polar angle together with the corresponding field integral. The product of the field integral and the momentum resolution is nearly independent of the polar angle, as expected. At a fixed momentum, this product depends only on the relative distance between the detectors and on their intrinsic resolution.

A comparison of the different reconstruction algorithms is presented on example of $e^{+}$emitted at $53^{\circ}$ (fig. 37 3right). Whereas the kick plane method is entirely dominated by the resolution of the META detectors $\left(\sigma_{M E T A}=6-13 \mathrm{~mm}\right.$ depending on polar angle), the curve for the Spline is used to provide a reasonable start value for the Runge-Kutta iterative fitting procedure.

As it has been stated above, the relative loss of resolution due to the deterioration of the overall chamber resolution is moderate for the electrons from the $\rho / \omega$ decay. The corresponding relative worsening of the $\mathrm{e}^{ \pm}$invariant mass resolution is of the order of $30 \%$ only. This has been confirmed by a direct measurement of the vector meson mass distribution. A preliminary analysis of 

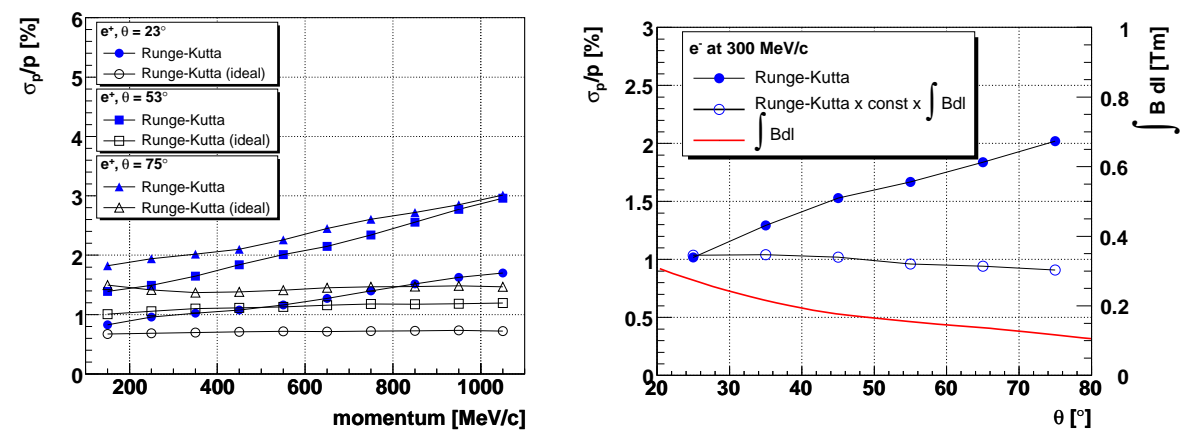

Figure 36: Left: Simulated momentum resolution for $\mathrm{e}^{+}$tracks with realistic errors shown for the 'Runge-Kutta' method (full symbols) and without applying any detector resolution ('Runge-Kutta ideal'; open symbols) for 3 polar angles. Right: Simulated resolution for $0.3 \mathrm{GeV} / \mathrm{c} \mathrm{e}^{+}$(full symbols) against angle. The product of the resolution and field integral (open symbols) directly shows that the momentum dependence on the polar angle is due to the variation of the integrated magnetic field (solid line). The right-hand scale displays the integrated magnetic field separately.

Figure 37: Simulated momentum resolution for $\mathrm{e}^{+}$tracks with realistic errors shown for different momentum reconstruction algorithms at a fixed polar angle of $53^{\circ}$.

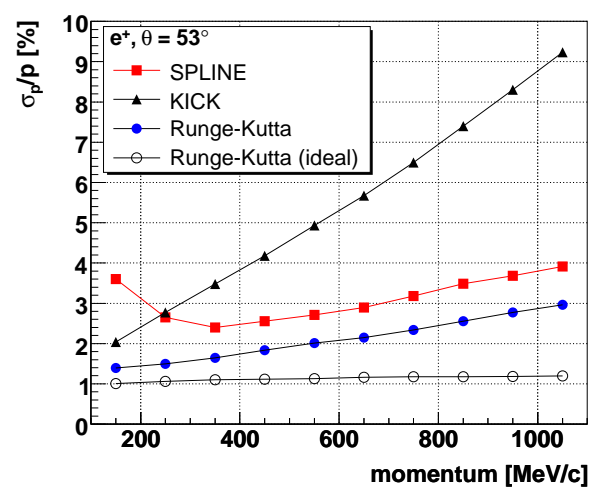

a recent experiment run on $\mathrm{p}+\mathrm{p}$ at $3.5 \mathrm{GeV}$ (see fig. 38) shows that the experimental peak width $\left(\sigma_{\omega}=21 \pm 2 \mathrm{MeV} / c^{2}\right)$ agrees quite well with the simulated one (solid histogram), dominated in this invariant mass region by the sum of 2 contributions, coming from $\omega$ (dashed histogram) and $\rho$ (dot-dashed histogram) two-body decays. One should also note that the peak position $(773 \pm 3) \mathrm{MeV} / \mathrm{c}^{2}$ is shifted by $9 \mathrm{MeV} / c^{2}$ as compared to the $\omega$ pole. This can be attributed to the electron energy loss in the target and the detector materials as shown by the GEANT simulations (solid histrogram).

\subsection{Particle identification}

\subsubsection{Overview}

HADES is primarily designed as a dielectron spectrometer, hence a main goal of the analysis is to achieve excellent electron-hadron separation over the 


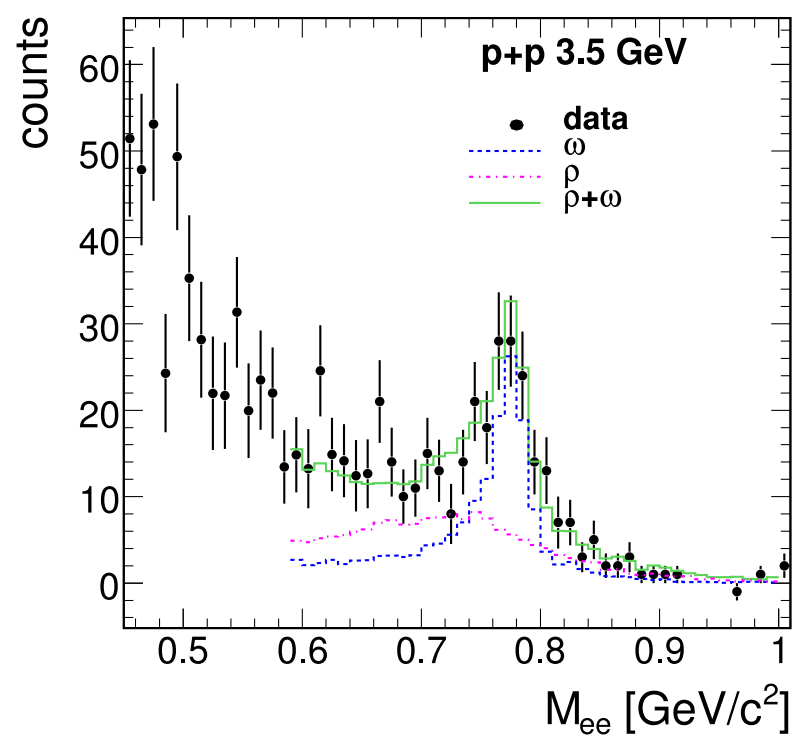

Figure 38: $\mathrm{e}^{+} \mathrm{e}^{-}$invariant mass spectrum in the $\omega$ region from $3.5 \mathrm{GeV}$ proton-proton reactions. In this invariant mass region, the dominant contributions of the $\rho$ (dot-dashed histogram) and of the $\omega$ (dashed histogram) are also indicated.

momentum range between 0.1 and $1 \mathrm{GeV} / \mathrm{c}$. However, for the normalization and interpretation of the dielectron data, a simultaneous measurement of hadrons $(\pi, \mathrm{K}, \mathrm{p})$ is mandatory as well. Therefore, much attention has been paid to the implementation of a universal Particle Identification (PID) method. Two different approaches have been employed for performing particle identification in HADES: (i) using a set of hard cuts on various observables, namely momentum, velocity, energy loss in TOF and MDC, hit patterns in RICH and Pre-Shower or (ii) applying a Bayesian method to those observables [65]. The basis of the latter one is a statistical test of the hypothesis that the reconstructed track properties are consistent with a given particle species. In practice, several observables from various sub-detectors are combined together to Probability Density Functions (PDF). These are determined for each observable and for all possible particle types in detailed simulations. The particle identification probability is then calculated based on the prior abundances of individual particle types, as well as on the specific PDFs of measured variables. Below we describe in detail the Bayesian approach.

\subsubsection{Description of the Method}

The Bayesian PID method is illustrated for the velocity-vs.-momentum relationship, referred to as the $\beta-p$ algorithm. Particle velocity $\beta$ (in units of the speed of light) and momentum $p$ are indeed the two observables that typically 
provide identification power, as illustrated in fig. 39. Well separated branches corresponding to positive and negative pions, protons and deuterons are visible.
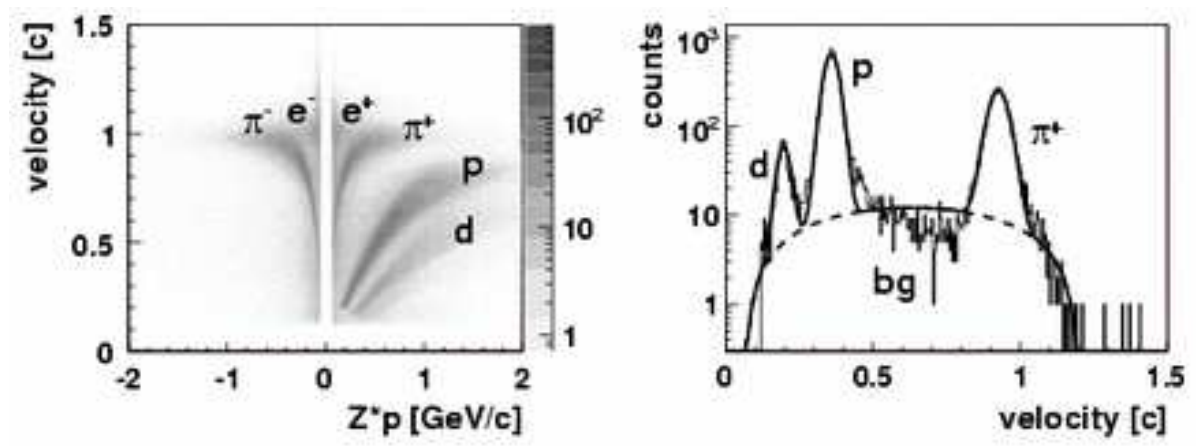

Figure 39: Left: Velocity vs. $Z p$ of charged particles detected with HADES in $\mathrm{C}+\mathrm{C}$ collisions at $2 \mathrm{AGeV}$ (note the logarithmic $\mathrm{z}$ scale). Right: Projection of $\beta$ vs. $Z p$ onto the velocity axis for positive particles with a momentum of $0.35 \mathrm{GeV} / \mathrm{c}$. Fits of PDFs are shown as well.

The first step in the Bayesian PID method consists in calculating the PDFs of the chosen observables for each particle type. For example, in case of the $\beta-p$ algorithm, these are obtained from the velocity distributions projected for various momentum and polar angle bins. The velocity distributions are fitted with a set of Gauss functions and a second order polynomial to describe signal and background, respectively (see fig. 39). The background term accounts for incorrectly reconstructed tracks. The fitted distributions are finally normalized to unity to be used as PDFs.

In a similar way, PDF distributions are created for other observables, e.g. for the energy loss signals from the TOF and TOFINO detectors, as well as from the MDC planes (see sect. 2.3.4) [59, 66]. More specifically, for electron identification in the $\mathrm{RICH}$ detector, various ring parameter distributions are used: ring radius, the number of contributing fired pads and their analog sum, and amplitude of the Pattern Matrix (see sect. 3.4). Likewise, electron-hadron separation in the Pre-Shower detector is based on a comparison of the integrated charge induced in consecutive layers of the Pre-Shower detector (see sect. 2.5.2).

The second step of the method consists in merging the probabilities from individual PID algorithms. For each particle type, the products of probabilities from all algorithms are calculated and the Bayes formula [65] is applied to take into account the relative particle abundances. With a set of $k$ independent experimental variables and $h$ being a particular particle hypothesis with known probability density function, the likelihood to observe the value of the discriminating variables for this particle hypothesis is given by

$$
L(\vec{x} \mid h)=\prod_{k} f_{k}\left(x_{k} \mid h\right)
$$


where $f_{k}\left(x_{k} \mid h\right)$ is the probability that a track with measurement $x_{k}$ is a particle of species $h=\mathrm{e}^{ \pm}, \pi^{ \pm}, \mathrm{K}^{ \pm}, \mathrm{p}, \mathrm{d}$.

The probability $P(h \mid \vec{x})$ that a given track corresponds to the particle type $h$ is then given by the Bayes theorem

$$
P(h \mid \vec{x})=\frac{L(\vec{x} \mid h) \mathcal{P}(h)}{\sum_{h=e^{ \pm}, \pi^{ \pm}, K^{ \pm}, p, d} L(\vec{x} \mid h) \mathcal{P}(h)},
$$

where $\mathcal{P}(h)$ is the probability to find particle $h$ for a given momentum $p$ and polar angle $\theta$ (relative abundance). The sum of $P(h \mid \vec{x})$ over all particle types $h$ is normalized to 1 . Knowing all $P(h \mid \vec{x})$ values for the track, the particle ID is assigned by selecting the particle type $h$ having the largest probability.

Two quality parameters, namely the PID efficiency and the PID purity, are evaluated to test the performance of the method (see e.g. [67] for details). They can be studied systematically in realistic simulations using heavy-ion collision events generated within the UrQMD transport model [68].

\subsubsection{PID performance}

A detailed analysis of the $\beta-p$ PID method reveals that $\pi^{+}$mesons can be separated from protons up to momenta of $p \simeq 1.0 \mathrm{GeV} / \mathrm{c}$ with a purity better than $80 \%$ and with an efficiency of almost $100 \%$. At higher momenta, the efficiency drops rapidly for $\pi^{+}$because of the rather limited TOFINO time resolution [69].

Kaon identification has been successfully demonstrated [70] over a momentum range $(150<p<800 \mathrm{MeV} / \mathrm{c})$ and for polar angles $\theta>45^{0}$ (TOF region) using combination of the $\beta-p$ method and the energy loss measurements in TOF and MDCs. After exchange of the TOFINO by the RPC [34] detector kaon identification in the full HADES acceptance will become possible.
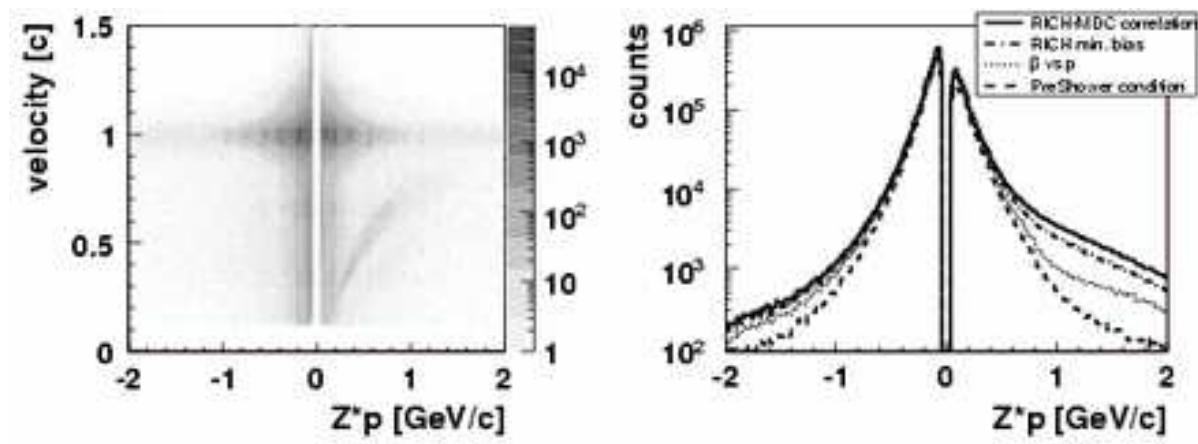

Figure 40: Left: Velocity vs. $Z p$ scatter plot for charged particle tracks correlated with a $\mathrm{RICH}$ ring. Data are from $2 \mathrm{AGeV} \mathrm{C}+\mathrm{C}$ collisions. Right: Projections on the $Z p$ axis, illustrating the effect of different electron identification conditions. 

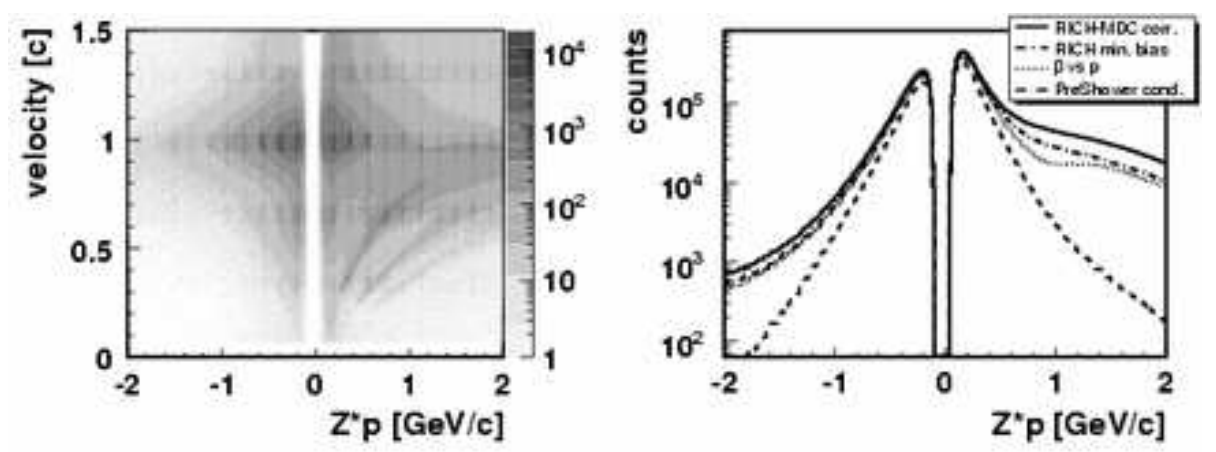

Figure 41: Same as fig. 40 but for $\mathrm{Ar}+\mathrm{KCl}$ collisions at $1.76 \mathrm{AGeV}$.

Below we present in more details electron identification which is the main goal of the HADES detector. Figure 40 left displays the same distribution as in fig. 39] but for LVL2-triggered events with an additional condition applied on the spatial correlation between electron tracks reconstructed in the RICH and MDC. Furthermore, only tracks involving spatially correlated hits, within a $2 \sigma$ window, on the TOF are shown.

Figure 40 right shows the projection of the two-dimensional $\beta$ vs. $Z p$ distribution and its reductions due to various electron identification conditions successively applied on: i) aforementioned track correlation in RICH-MDC ii) ring quality like ring radius, number of contributing pads per ring (see sect. 2.2.4) iii) $\beta-p$ correlation and iv) Pre-shower electron signature (see sect. 2.5.2). A detailed investigation of measured electron distributions and dedicated $\mathrm{C}+\mathrm{C}$ Monte Carlo simulations proved that the residual contamination of hadronic background in the final electron sample is less than $3 \%$ and contributes mostly at higher momenta $(p>0.6 \mathrm{GeV} / \mathrm{c})$. The efficiency of the PID method is close to $80 \%$ and drops to $70 \%$ with higher positron and electron momenta. These results depend to some degree on the particular reaction investigated. Figure 41 shows the case of $1.76 \mathrm{AGeV} \mathrm{Ar}+\mathrm{KCl}$ collisions, with approximately 5 times larger track multiplicity. A significantly larger intensity of positive charged tracks, correlated with RICH rings as compared to negative ones, indicates an increased contribution from misidentified protons. However, using Pre-Shower electron conditions, a clean lepton identification up to $p=1 \mathrm{GeV} / \mathrm{c}$ could be achieved.

\subsection{Pair reconstruction}

\subsubsection{Overview}

In contrast to Compton scattering of high energy photons and weak decays of muons, all other reactions and decay processes create correlated electronpositron pairs. The main aim of the HADES analysis is to reconstruct with high 
accuracy the dielectron invariant mass from the observed electron and positron three-momenta. Hence, in the next major step of the data analysis, identified leptons are combined into unlike-sign $\left(\mathrm{e}^{+} \mathrm{e}^{-}\right)$and like-sign $\left(\mathrm{e}^{+} \mathrm{e}^{+}, \mathrm{e}^{-} \mathrm{e}^{-}\right)$lepton pairs. In this way, all pair combinations are reconstructed, the correlated true pairs as well as uncorrelated pairs, constituting the so-called Combinatorial Background (CB). At beam energies available at the SIS, most of the CB arises from photon conversion $(\sim 60 \%)$, either in the target or in the RICH radiator gas, and from Dalitz decays of the ubiquitous $\pi^{0}$ mesons $(\sim 25 \%)$. Both sources lead to pairs with predominantly small opening angles, the so-called close pairs with typically $\alpha_{e^{+} e^{-}}<10^{\circ}$.

The acceptance of the spectrometer for pairs is shown as a function of the pair invariant mass and of the transverse momentum in fig. 42. It is determined by the detector geometry and the deflection in the magnetic field for both $\mathrm{e}^{+}$and $\mathrm{e}^{-}$as a function of the particle charge $Z$, momentum $p$ and angles $\theta$ and $\phi$. The geometrical pair acceptance of HADES is obtained as a product of the two singleelectron acceptances (see fig. 42), which together with the momentum resolution function constitute the HADES acceptance filter (available upon request from the authors).

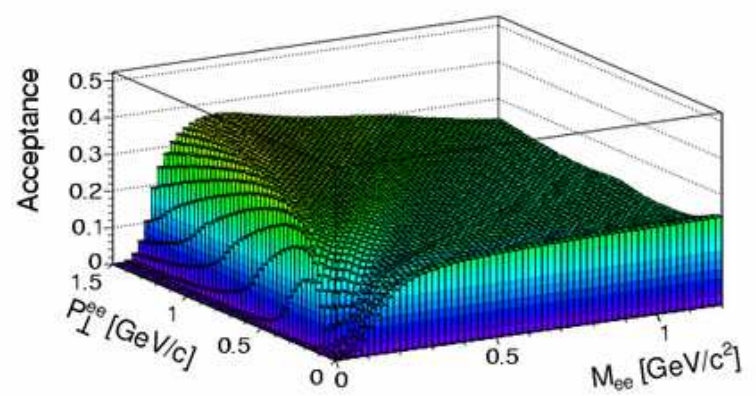

Figure 42: Geometrical acceptance for $\mathrm{e}^{+} \mathrm{e}^{-}$pairs with opening angle $\alpha_{e^{+} e^{-}}>9^{\circ}$ as a function of pair mass and transverse momentum. The acceptance is averaged over a distribution in rapidity in the interval $[0,2]$ representative of the reactions at SIS energies.

\subsubsection{Rejection of close pairs}

Fortunately, close pairs with very small opening angle leading to overlapping tracks in the tracking system can be rejected efficiently by applying a condition on the $\chi^{2}$ of track segment fits reconstructed in the analysis. From simulations we observe that this cut rejects more than $90 \%$ of close pairs with opening angles $\alpha_{e^{+} e^{-}}<3^{\circ}$, while still achieving an efficiency of $95 \%$ for single electron tracks. The number of uncorrelated pairs is further reduced by applying a cut on 
the pair opening angle (typically $\alpha_{e^{+} e^{-}}>9^{\circ}$ ) and by rejecting pairs which share common hits in the inner or the outer detector parts. In addition, both legs of pairs rejected by either one of these two cuts are marked and are not allowed to contribute to the pair sample retained for further physics analysis. Those pairs are used to create pair observable distributions, like the pair invariant mass, the pair transverse momentum and the pair rapidity. Likewise, the combinatorial background of uncorrelated pairs is determined from this pair sample to which all further operations described below are applied.

\subsubsection{Combinatorial background}

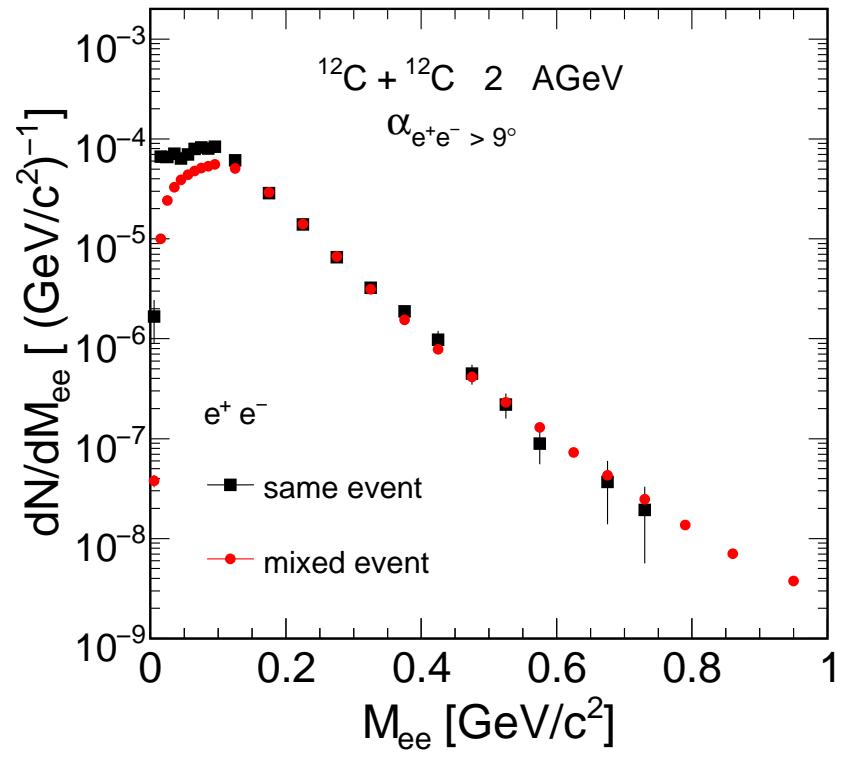

Figure 43: Comparison of the like-sign pair technique and the mixed-event one for the combinatorial background, properly normalized to each other in the intermediate-mass region $0.15-0.5 \mathrm{GeV} / \mathrm{c}$.

The combinatorial background of remaining uncorrelated pairs has to be modeled in order to be able to subtract it from the total $\mathrm{e}^{+} \mathrm{e}^{-}$yield. Commonly two strategies can be followed [71] to build up the CB spectrum: (i) the like-sign pair technique and (ii) the mixed-event technique. In the first method, like-sign $\mathrm{e}^{+} \mathrm{e}^{+}$and $\mathrm{e}^{-} \mathrm{e}^{-}$pairs are formed and subjected to the same selection criteria as the unlike-sign pairs. From the reconstructed like-sign invariant-mass distributions $\mathrm{dN}^{++} / d M_{e e}$ and $\mathrm{dN}^{--} / d M_{e e}$, the respective $\mathrm{CB}$ distribution is obtained as their geometric mean $d N_{C B} / d M_{e e}=2 \sqrt{d N^{++} / d M_{e e} \cdot d N^{--} / d M_{e e}}$. The signal distribution is calculated by subtracting the CB from the total $\mathrm{e}^{+} \mathrm{e}^{-}$yield, 
i.e. $\mathrm{dN}^{+-} / d M_{e e}-\mathrm{dN}_{C B} / d M_{e e}$. In the same way, this method can be applied to other pair observables, e.g. the pair rapidity or transverse momentum.

The advantage of the like-sign pair technique is that it naturally includes residual correlations due to total energy and charge conservation, as well as collective flow patterns, but its statistical accuracy is limited and may not be sufficient in certain kinematical regions. Here, the mixed-event technique can help. A pair distribution is then built by combining two tracks from different events which are inherently uncorrelated. This method offers a large number of mixed combinations even for small event samples. We also make sure that only tracks from the same event class are combined, i.e. (i) they originate from the same target segment and (ii) they belong to the same track-multiplicity bin. Finally, we build mixed-event $\mathrm{e}^{+} \mathrm{e}^{-}$pair distributions and normalize them to the integral of the corresponding like-sign distributions within statistically significant and correlation-free ranges of the like-sign distributions (see fig. 43).

As one can see the mixed background agrees very well with the like-sign CB above $M_{e e}>150 \mathrm{MeV} / \mathrm{c}^{2}$. At lower masses both distributions substantially differs. It is due to already mentioned correlations between conversion pairs stemming from both photons originating from the $\pi^{0} \rightarrow \gamma \gamma$ decay process. For this reason at low masses we use like-sign background which,as it has been checked by simulations, properly describes spectral shape of the CB.
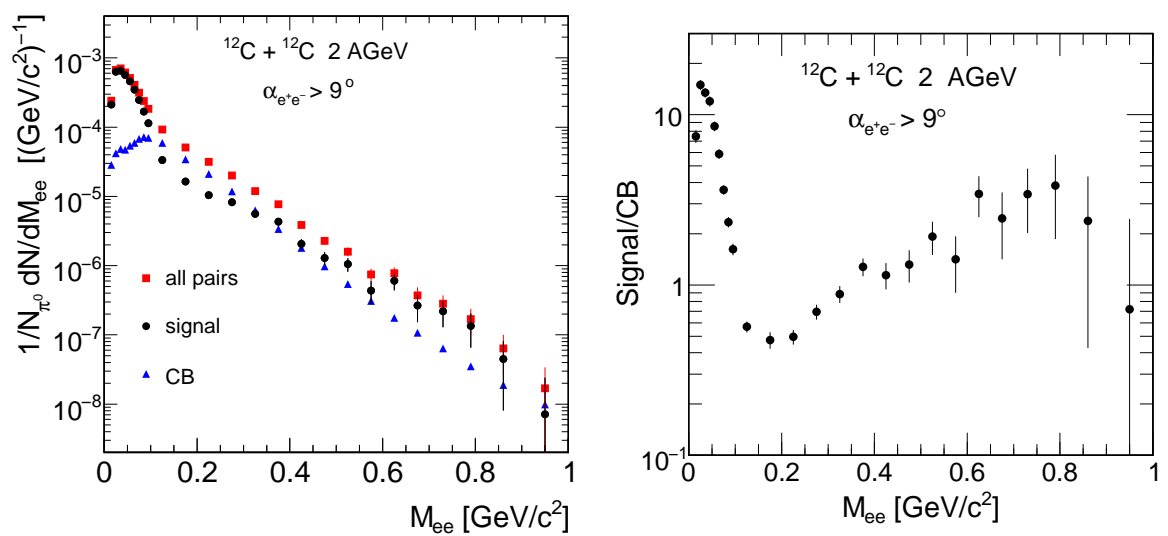

Figure 44: Left: $\mathrm{e}^{+} \mathrm{e}^{-}$invariant mass spectrum for the $\mathrm{C}+\mathrm{C}$ reaction at $2 \mathrm{AGeV}$. The signal (full circles) is obtained after subtraction of the combinatorial background (full triangles) from the all pairs signal (full squares) as explained in the text. Right: Signal to combinatorial background ratio as a function of the dilepton invariant mass in the hard-cut analysis of the same data sample.

Figure 44 illustrates the signal-to-combinatorial background ratio observed in $\mathrm{C}+\mathrm{C}$ collisions at $2 \mathrm{AGeV}$. A good ratio, about a factor two in the intermediateand high-mass region, directly reflects a small conversion contribution, being a consequence of the concepts used for the RICH construction - short path length and thin mirror - and for the tracking system detectors which are made of low-Z 

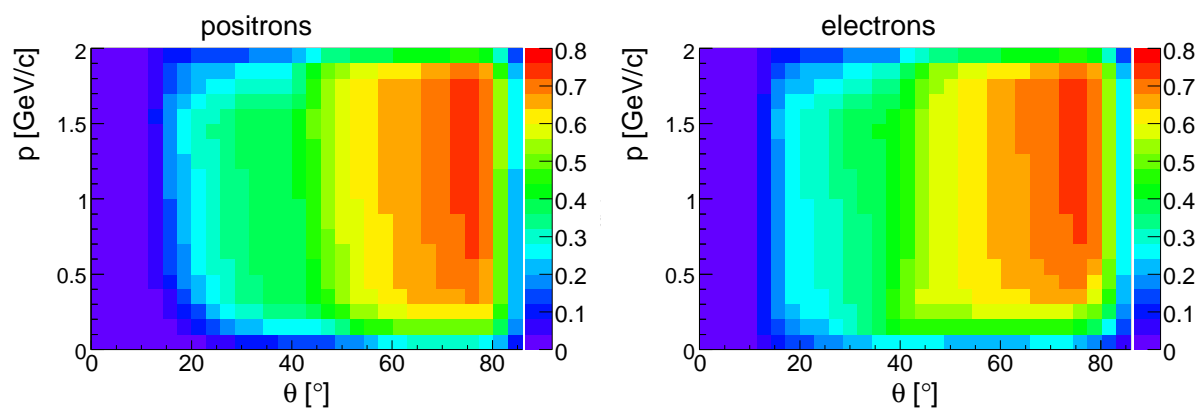

Figure 45: Left: Positron combined detection and reconstruction efficiency, averaged over the azimuthal angle. Right: Same but for the electron. The geometrical acceptance together with the bending and focusing effects of the magnetic field explains the slight different behavior between the electron and the positron efficiencies.

material such as He-based counting gas and $\mathrm{Al}$ wires.

\subsubsection{Efficiency corrections}

We correct the spectra for detector and reconstruction inefficiencies by MonteCarlo simulations embedding electron tracks with uniform momentum and isotropic angular distributions into A + A events, either generated with the UrQMD transport model or taken from measured data. The embedded tracks are further digitized and processed through the same analysis chain as the measured data. The single-electron efficiencies, $\epsilon_{-}(z, p, \theta, \phi)$ and $\epsilon_{+}(z, p, \theta, \phi)$, are then deduced. Values integrated over the azimuthal angle are shown in fig. 45 for both electrons and positrons.

The data are then corrected on a pair-by-pair basis with the weighting factor $1 / E_{+-}$, with $E_{+-}=\epsilon_{+} \cdot \epsilon_{-}$for given electron momenta and emission angles, $E_{+-}$ranging typically from $10 \%$ at $M_{e e}=0.1 \mathrm{GeV} / \mathrm{c}^{2}$ to $20 \%$ at $M_{e e}=$ $1 \mathrm{GeV} / \mathrm{c}^{2}$. The $\mathrm{CB}$ is treated likewise and subtracted, as described above, to obtain the efficiency-corrected pair signal distribution. This prescription relies on the assumption that the single-leg efficiencies are independent, as was carefully checked in our simulations and proven to be valid within $15 \%$ for pairs with opening angles $\alpha_{e+e-}>9^{\circ}$. 


\section{Conclusion}

We have presented a description of the High-Acceptance Dielectron Spectrometer HADES installed at the SIS facility in GSI Helmholtzzentrum für Schwerionenforschung. Given its hexagonal structure, HADES is nearly azimuthally symmetric and covers a polar angular range from $18^{\circ}$ to $85^{\circ}$. The dedicated trigger system permits the measurements of dielectron spectra in reasonably short beam times. Having this setup at our disposal, a rich physics program ranging from hadron to heavy-ion physics is in progress with unprecedented statistics and mass resolution. Actually, HADES was used for data taking in $\mathrm{p}+\mathrm{p}, \mathrm{d}+\mathrm{p}, \mathrm{p}+\mathrm{Nb}, \mathrm{C}+\mathrm{C}$ and $\mathrm{Ar}+\mathrm{KCl}$ reactions at various beam energies. First physics results on $\mathrm{e}^{+} \mathrm{e}^{-}$invariant mass spectra in $\mathrm{C}+\mathrm{C}$ collisions at 1 and $2 \mathrm{AGeV}$ have been published [72, 73].

The detector components and their respective performances have been described in detail. Emphasis in electron identification is put on the Ring Imaging Cherenkov counter. Additional electron selectivity is gained from the PreShower and TOF detectors which finally results in a very good purity. The tracking system, consisting of two layers of multi-wire drift chambers in front of a superconducting magnet and two layers behind it, allows for momentum determination of charged particles including also charged hadrons. The performance for dilepton decays of the light vector mesons enables a clear identification of $\rho$ and $\omega$ decays as well as the investigation of predicted medium modifications of spectral properties such as mass or width. Moreover, combination of measurements of the energy loss in the MDCs and in the TOF detector, of the velocity and of the momentum in the tracking system allows for pion, kaon and proton separation, important for the reaction characterization and normalization.

There is a large variety of theoretical predictions of dielectron spectra ranging from elementary $\mathrm{p}+\mathrm{p}$ and $\mathrm{p}+\mathrm{n}$ channels [74] to heavy-ion collisions [75]. These predictions, differing in many details, can now be verified or falsified by measurements with HADES. Indeed, theory-based interpretations [76] of our spectra [72, 73] show the strong interest in dielectron spectroscopy as a tool for understanding hadronic reactions, per se or as a part of heavy-ion collisions, in the non-perturbative domain. The motivation can be extended to the quest of medium-modified hadron properties and the origin of the masses of the hadrons. To accomplish these goals, the large geometrical acceptance of the spectrometer has been designed which results in reduced statistical and systematic errors and enables the investigation of exclusive elementary reactions channels induced by protons or pions. The overall detector design has been optimized with respect to low photon conversion probabilities in order to minimize the combinatorial background. The apparatus is ready for extending the employed projectiles to pions, aimed at studying pion-induced dilectron emission off protons, deuterons and heavy target nuclei. These reactions are helpful in disentangling the numerous channels contributing to the complex spectra in heavy-ion collisions. Interesting theoretical predictions have been made [77] which await experimental verification. While dielectron spectroscopy is the primary goal of HADES, the precise tracking system allows also for the investigation of other rare probes 
in particular those which contain strange quarks.

After having exploited the opportunities at SIS18, HADES is foreseen to operate at SIS100 within FAIR, thus extending dilepton spectroscopy in a hitherto unexplored region of beam energies. 


\section{Acknowledgments}

The authors are grateful to all national funding agancies. This work was supported in part by grants from BMBF (TM872 I TP2, 06MT190 TP2, 06F 140 and 06DR135), DFG, GSI (TM Koe1K, TM FR1 and TM KR2), Czech Republic (MSMT LC07050 and LA316, GAASCR IAA100480803), EU 6th Framework Program (RII3-CT-2004-506078 and RII3-CT-2005-515876), by MLL München, by the Spanish Funding Agency (FPA2000-2041-C02-02), by INTAS (94-1233, 96-0468, 06-1000012-8861), by 528/92/LN Stiftung für Deutsch-Polnische Zusammenarbeit KBN 5P03B 140 20, by CNRS/IN2P3 and by PGIDT02PXIC20605PN, FPA2003-7581-C02-02. 


\section{References}

[1] R. Rapp and J. Wambach, Adv. Nucl. Phys. 25 (2000) 1.

[2] R. Schicker et al., Nucl. Instr. Meth. A380 (1996) 586.

[3] V. Metag, Prog. Part. Nucl. Phys. 30 (1993) 75.

[4] P. Salabura et al., Prog. Part. Nucl. Phys. 53 (2004) 49.

[5] J. Friese et al., Prog. Part. Nucl. Phys. 42 (1999) 235.

[6] A. Yegneswaran et al., Nucl. Instr. Meth. A290 (1990) 61.

[7] TOSCA part of OPERA 2D and 3D, Vector Fields, UK, www.vectorfields.com.

[8] Linde Kryotechnik AG, Daettlikonerstrasse 5, PO-Box, CH-8422 Pfungen, Switzerland.

[9] K. Zeitelhack et al., Nucl. Instr. Meth. A433 (1999) 201.

[10] J. Friese et al., Nucl. Instr. Meth. A438 (1999) 86.

[11] A. Kastenmüller et al., Nucl. Instr. Meth. A433 (1999) 438.

[12] J. Friese et al., Nucl. Instr. Meth. A502 (2003) 241.

[13] J. Friese et al., PEGASUS, the RICH gas system description and manual (2005).

[14] J. Lehnert et al., Nucl. Instr. Meth. A502 (2003) 261.

[15] W. Beusch et al., Internal Note 4, CERN/ECP/MIP (1993).

[16] J. Lehnert et al., Nucl. Instr. Meth. A433 (1999) 268.

[17] R. Dübgen, G. Popp, Z. Werkstofftechnik 15 (1984) 331.

[18] P. Maier-Komor et al., Nucl. Instr. Meth. A438 (1999) 152.

[19] P. Maier-Komor et al., Nucl. Instr. Meth. A480 (2002) 65.

[20] L. Fabbietti et al., Nucl. Instr. Meth. A502 (2003) 256.

[21] C. Garabatos et al., Nucl. Instr. Meth. A412 (1998) 38.

[22] H. Bokemeyer et al., Nucl. Instr. Meth. A477 (2002) 397.

[23] C. Müntz et al., Nucl. Instr. Meth. A535 (2004) 242.

[24] K. Kanaki et al., IEEE Trans. Nucl. Sci. 51 (2004) 939. 
[25] C. Lippmann, Aufbau und Inbetriebnahme eines Gasqualitätsmonitors für die HADES Driftkammern, Diploma thesis, Johann Wolfgang GoetheUniversität Frankfurt, 2000.

[26] E. Badura et al., Particles and Nuclei, Letters 1 (2000) 73.

[27] M. Newcomer, IEEE Trans. Nucl. Sci. 40 (1993) 630.

[28] J. Markert. Proceedings of the XL International Winter Meeting of Nuclear Physics, Bormio, (2002) p. 123.

[29] A. Abashian et al., Nucl. Instr. Meth. A479 (2002) 117.

[30] B. Aubert et al., Nucl. Instr. Meth. A479 (2002) 1.

[31] W.M. Yao et al., Review of Particle Properties, section 'Particle detectors', Journal of Physics G 33 (2006) 1.

[32] C. Agodi et al., Nucl. Instr. Meth. A492 (2002) 14.

[33] F. Krizek, Study of inclusive electron-positron pair production of $\mathrm{Ar}+\mathrm{KCl}$ at 1.756 AGeV, Ph.D. thesis, Czech Technical University Prague, 2008.

[34] H. Alvarez-Pol et al., Nucl. Instr. Meth. A535 (2004) 277.

[35] S. Spataro, Characterization of the HADES Spectrometer in pp Collisions at 2.2 GeV: Elastic Scattering and Exclusive $\eta$ Reconstruction, Ph.D. thesis, Università degli Studi di Catania, 2002.

[36] G.D. Alekseev et al., Nucl. Instr. Meth. 177 (1980) 385.

[37] M. Atac et al., Nucl. Instr. Meth. 200 (1982) 345.

[38] A. Balanda et al., Nucl. Instr. Meth. A531 (2004) 445.

[39] A. Balanda et al., Nucl. Instr. Meth. A417 (1998) 360.

[40] GEANT3 Detector description and simulation tool, CERN long writeup W5013 (1993).

[41] R.S. Simon et al., Progr. Part. Nucl. Phys. 42 (1999) 247.

[42] J. Díaz et al., Nucl. Instr. Meth. A478 (2002) 511.

[43] E. Berdermann et al., Diamond and Related Materials 10 (2001) 1770.

[44] M. Traxler et al., IEEE Trans. Nucl. Sci. 47 (2000) 376.

[45] M. Traxler, Real time dilepton selection for the HADES spectrometer, Ph.D. thesis, Justus-Liebig-Universität Gießen, 2001.

[46] J. Lehnert, Echtzeit-Mustererkennung zum Elektronennachweis mit einem RICH-Detektor in relativistischen Schwerionenkollisionen, Ph.D. thesis, Justus-Liebig-Universität Gießen, 2000. 
[47] I. Fröhlich et al., IEEE Trans. Nucl. Sci. 55 (2008) 59.

[48] A. Akindinov et al., Nucl. Instr. Meth. A533 (2004) 178.

[49] A. Toia et al., Nucl. Instr. Meth. A502 (2003) 270.

[50] http://www.aps.anl.gov/epics/index.php.

[51] M. Sánchez, Momentum reconstruction and pion production analysis in the HADES spectrometer at GSI, Ph.D. thesis, University of Santiago de Compostela, 2003.

[52] R. Brun and F. Rademakers, ROOT, an object-oriented data analysis framework, Nucl. Instr. Meth. A389 (1997) 81.

[53] Oracle Corporation, 500 Oracle Parkway, Redwood Shores, CA 94065, USA.

[54] The Qt class library, Trolltech AS, PO Box 4332 Oslo, Norway.

[55] M. Kagarlis, Pluto ${ }^{++}$, a Monte-Carlo simulation tool for hadronic physics, GSI internal report 2000-03, unpublished.

[56] I. Fröhlich et al., XII International Workshop on Advanced Computing and Analysis Techniques for Physics Research, PoS 076 (2008), see also arXiv:nucl-ex/0708.2382.

[57] G. Agakichiev, V. Pechenov et al., Particle and Nuclei, Letters 2 (2000) 1001.

[58] Garfield, Simulation of gaseous detectors, http://www.cern.ch/garfield, Online User Guide.

[59] J. Markert, Untersuchung zum Ansprechverhalten der VieldrahtDriftkammern niedriger Massenbelegung des HADES Experiments, Ph.D. thesis, Johann Wolfgang Goethe-Universität Frankfurt, 2005.

[60] A. Ierusalimov, HADES internal report (2002).

[61] A. Rustamov, Exclusive $\eta$ Meson Reconstruction in Proton-Proton Collisions at 2.2 GeV with the HADES Spectrometer and High Resolution Tracking, Ph.D. thesis, Technische Universität Darmstadt, Darmstadt, 2006.

[62] H. Wind, Nucl. Instr. Meth. 115 (1974) 431.

[63] W. H. Press, S. A. Teukolsky, W. T. Vetterling, and B. P. Flannery, "Numerical Recipes", $3^{\text {rd }}$ Edition, Cambridge University Press (2007).

[64] A. Sadovsky, Investigation of $K^{+}$meson production in $C+C$ collisions at 2 AGeV with HADES, Ph.D. thesis, Technische Universität Dresden, ISSN 1437-322X, 2007. 
[65] R. Barlow et al., Recommended Statistical Procedures for BABAR, BABAR analysis document, unpublished. No. 318 (2002), www.slac.stanford.edu/BFROOT/www/Statistics/Report/report.pdf.

[66] A. Schmah et al., International Nuclear Physics Conference, Tokyo, 2007.

[67] B. Hommez, Nucl. Instr. Meth. A502 (2003) 294.

[68] S. Bass et al., Prog. Part. Nucl. Phys. 41 (1998) 225.

[69] G. Agakichiev et al., arXiv:nucl-ex/0902.4377v1 and submitted to EPJA.

[70] G. Agakichiev et al., arXiv:nucl-ex/0902.3487v1 and submitted to PRC.

[71] P. Crochet and P. Braun-Munzinger, Nucl. Instr. Meth. A484 (2002) 564.

[72] G. Agakichiev et al., Phys. Rev. Lett. 98 (2007) 052302.

[73] G. Agakichiev et al., Phys. Lett. B663 (2008) 43.

[74] R. Shyam, U. Mosel, Phys. Rev. C67 (2003) 065202. L.P. Kaptari, B. Kämpfer, Nucl. Phys. A764 (2006) 338. E.L. Bratkovskaya, W. Cassing, M. Effenberger, U. Mosel, Nucl. Phys. A653 (1999) 301. C. Ernst, S.A. Bass, M. Belkacem, H. Stöcker, W. Greiner, Phys. Rev. C58 (1998) 447. B. Kämpfer, A.I. Titov, E.L. Bratkovskaya, Phys. Lett. B301 (1993) 123. B. Kämpfer, A.I. Titov, E.L. Bratkovskaya, Phys. Rev. C51 (1995) 227.

[75] A.I. Titov, B. Kämpfer, Phys. Rev. C76 (2007) 065211; M.D. Cozma, C. Fuchs, E. Santini, A. Faessler, Phys. Lett. B640 (2006) 170. C. Fuchs, A. Faessler, Prog. Part. Nucl. Phys. 53 (2004) 59. K. Shekhter, C. Fuchs, A. Faessler, M. Krivoruchenko, B. Martemyanov, Phys. Rev. C68 (2003) 014904. M.F.M. Lutz, G. Wolf, B. Friman, Nucl. Phys. A706 (2002) 431. W. Cassing, E.L. Bratkovskaya, Phys. Rept. 308 (1999) 65. W. Cassing, E.L. Bratkovskaya, R. Rapp, J. Wambach, Phys. Rev. C57 (1998) 916. E.L. Bratkovskaya, W. Cassing, Nucl. Phys. A619 (1997) 413. T.I. Gulamov, A.I. Titov, B. Kämpfer, Phys. Lett. B372 (1996) 187. A.I. Titov, T.I. Gulamov, B. Kämpfer, Phys. Rev. D53 (1996) 3770. E.L. Bratkovskaya, W. Cassing, U. Mosel, Phys. Lett. B376 (1996) 12. E.L. Bratkovskaya, W. Cassing, U. Mosel, O.V. Teryaev, A.I. Titov, V.D. Toneev, Phys. Lett. B362 (1995) 17.

[76] K. Schmidt, E. Santini, S. Vogel, C. Sturm, M. Bleicher, H. Stöcker, arXiv:nucl-th/0811.4073. E. Santini, M.D. Cozma, A. Faessler, C. Fuchs, M.I. Krivoruchenko, B. Martemyanov, arXiv:nucl-th/0811.2065. E. Santini, M.D. Cozma, A. Faessler, C. Fuchs, M.I. Krivoruchenko, B. Martemyanov, Phys. Rev. C78 (2008) 034910. E.L. Bratkovskaya, W. Cassing, Nucl. Phys. A807 (2008) 214. M. Thomere, C. Hartnack, Gy. Wolf, J. Aichelin, Phys. Rev. C75 (2007) 064902. H.W. Barz, B. Kämpfer, Gy. Wolf, M. Zetenyi, arXiv:nucl-th/0605036. 
[77] M.F.M. Lutz, B. Friman, M. Soyeur, Nucl. Phys. A713 (2003) 97. B. Kämpfer, A.I. Titov, B.L. Reznik, Nucl. Phys. A721 (2003) 583. A. Faessler, C. Fuchs, M.I. Krivoruchenko, B.V. Martemyanov, J. Phys. G29 (2003) 603. A.I. Titov, B. Kämpfer, Eur. Phys. J. A12 (2001) 217. A.I. Titov, B. Kämpfer, B.L. Reznik, Eur. Phys. J. A7 (2000) 543. M. Effenberger, E.L. Bratkovskaya, W. Cassing, U. Mosel, Phys. Rev. C60 (1999) 027601. T. Weidmann, E.L. Bratkovskaya, W. Cassing, U. Mosel, Phys. Rev. C59 (1999) 919. 